Check for updates

Cite this: J. Mater. Chem. A, 2020, 8 , 11075

Received 19th February 2020

Accepted 1st April 2020

DOI: 10.1039/d0ta01973a

rsc.li/materials-a

\section{Polymeric carbon nitrides and related metal-free materials for energy and environmental applications}

\begin{abstract}
Jesús Barrio, (D) Michael Volokh (D) and Menny Shalom (DD*
Carbon nitride polymers have emerged as a new class of materials for a wide range of applications such as photo- and electro-catalysis, sensors, bioimaging and more due to their chemical, photophysical and catalytic properties as well as their low-price, facile synthesis and high stability under harsh chemical conditions. In this review we begin with a broad overview of carbon-based materials, arriving at the focus of this review, polymeric carbon nitrides (CNs). After a brief overview of applications, we delve into their various synthetic methods, with an emphasis on achieving control on the nanoscale features of this intriguing polymeric semiconductor. The main synthetic pathways include copolymerization at various stages, templating, an ionothermal pathway and harnessing of supramolecular pre-organization, which are discussed in detail along with $\mathrm{CN}$ growth and deposition on substrates. Finally, we give our perspectives on the evolution of this field, the current limitations, and elaboration of achievable control over the chemical composition, the electronic structure and the morphology of this family of materials
\end{abstract}

Department of Chemistry and Ilse Katz Institute for Nanoscale Science and Nanotechnology, Ben-Gurion University of the Negev, Beer-Sheva 8410501, Israel. E-mail: mennysh@bgu.ac.il

\section{Carbon materials: synthesis, applications and challenges}

Carbon materials ranging from graphene, ${ }^{1}$ carbon nanotubes $(\mathrm{CNTs})^{2}$ or fullerenes ${ }^{3}$ to activated carbon and aerogels ${ }^{4}$ are widely used for environmental and energy-related purposes ${ }^{5-8}$ such as batteries, ${ }^{9,10}$ supercapacitors ${ }^{11}$ and electrocatalysis ${ }^{12,13}$

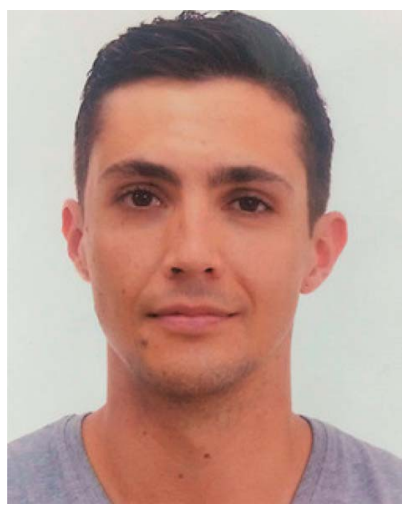

Jesús Barrio received his B.Sc in Chemistry from the Universidad Autónoma de Madrid (Madrid, Spain) in 2014 and in 2016 his M.Sc in Nanoscience and Molecular Nanotechnology while working at the IMDEA Nanoscience Institute. He then carried out a research project at the Max Planck Institute for Colloids and Interfaces where he developed a keen interest in the design of carbon nitride materials and their application in photocatalysis. Afterwards, he moved along with his PhD supervisor, Prof. Menny Shalom, to the Chemistry Department of the Ben-Gurion University of the Negev where he is currently finishing his doctoral studies. His interests cover the design of low dimensional materials and hybrid structures for energy-related applications.

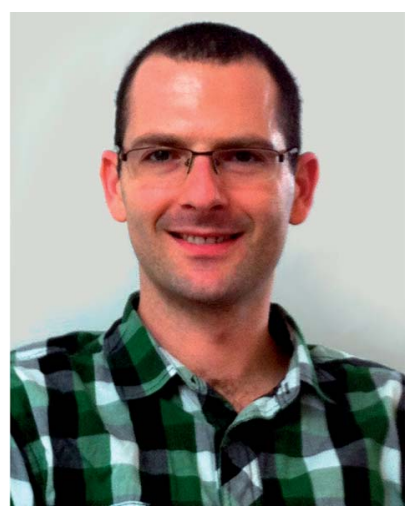

Michael Volokh received his BSc in Chemistry and Chemical Engineering from the Ben-Gurion University of the Negev (Israel). During his MSc and PhD (2017), under the supervision of Prof. Taleb Mokari, he focused on gas- and liquid-phase synthesis and characterization of inorganic hybrid nanostructures. His current research interests include photoelectrocatalysis, the formation of complex hybrid nanostructures, and electrophoretic deposition. 
due to their conductivity, nanosized-derived effects (e.g., dirac points in graphene, metallic or semiconductor CNTs and electron accepting properties of fullerenes) and the possibility of inducing defects by chemical design. ${ }^{14}$ There are numerous synthetic pathways to synthetize this class of materials, such as chemical vapor deposition (CVD) ${ }^{15-17}$ pyrolysis of C-rich molecules, ${ }^{\mathbf{1 8 - 2 0}}$ hydrothermal means, etc. ${ }^{21-25}$ Nevertheless, in most catalysis and energy-related scenarios, these materials serve as a support or as a platform for an additional active component or molecular complex to perform a given reaction. ${ }^{26}$ The morphology and properties of the final carbon-based material strongly depend on the synthetic pathway, as well as on the precursor. ${ }^{27}$ Additionally, utilizing foreign elements like templating agents or doping species ${ }^{28-30}$ can precisely tune the porous structure and electronic configuration, towards their implementation in a wider range of applications..$^{31,32}$

\section{Inserting heteroatoms into carbon matrices}

To modify the electronic structure of C-materials and enable semiconductor-like properties, such as UV/visible light absorption, the formation of defects through the insertion of heteroatoms has emerged as a promising approach. Chemical elements that differ in parameters like the atomic radius or electronegativity can induce effects on the materials that are beneficial for their application in energy-related fields, i.e., the creation of a band gap or selectivity towards a given reaction. Furthermore, combining several heteroatoms in a Cmatrix can produce synergistic effects such as the formation of reactive centers and a change in its intrinsic mechanical, chemical, physical and electronic properties. ${ }^{29}$ The insertion of nitrogen into a carbonaceous matrix is among the most frequently reported methods, given the straightforward synthetic pathways for such an insertion; for example, a thermal treatment of precursors containing $\mathrm{C}-\mathrm{N}$ or a hydrothermal synthesis. Nevertheless, other heteroatoms, such as B, P, S, or metals, display a better affinity towards

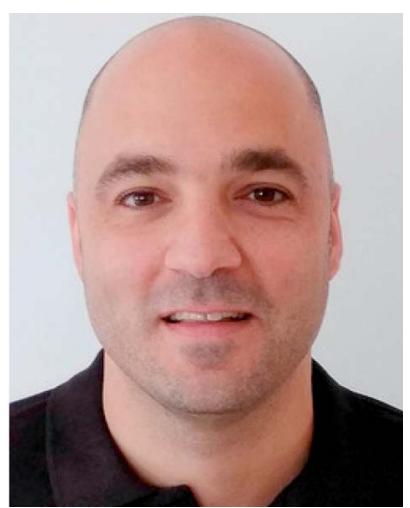

Prof. Menny Shalom received his PhD from Bar Ilan University, Israel. For his postdoctoral research he joined the Max Planck Institute of Colloids and Interfaces (MPI), Germany, as a Minerva fellowship fellow. From 2013 to 2016 he was a group leader at the MPI and since the end of 2016 he has been an Associate Professor at the Ben-Gurion University of the Negev, Israel. His group is focused on the synthesis of new materials for photo-electrochemical cells and as electrocatalysts for water splitting. reactant molecules for various chemical reactions and therefore their inclusion within C-materials has been a common subject of research. ${ }^{33}$

Non-metallic elements have been typically included by a co-polymerization route from a C-source with heteroatomcontaining molecules at high temperatures or by direct thermal calcination of (bio)molecules, such as amino acids and ionic liquids. ${ }^{34-38}$ Wohlgemuth et al. obtained carbon microspheres and aerogels co-doped with nitrogen and sulfur by the hydrothermal treatment of glucose along with amino acids containing sulfur, and they studied their electrochemical performance for the oxygen reduction reaction (ORR). ${ }^{39,40}$ Furthermore, S-doped C-materials were recently explored for the nitrogen reduction reaction (NRR); Xia et al. prepared S-doped carbon nanospheres by a hydrothermal reaction comprising glucose and benzyl disulfide. The resulting materials showed excellent selectivity and a faradaic efficiency of $7.47 \%$ at $-0.7 \mathrm{~V}$ versus (vs.) RHE, much higher than the undoped material $(1.45 \%) .{ }^{41}$ The presence of heteroatoms within carbon materials strongly affects their reactivity in fields such as electrocatalysis, batteries or supercapacitors. B and $\mathrm{P}$, for example, display a lower electronegativity than carbon, which results in a charge redistribution and modifies the adsorption modes of reactant molecules. ${ }^{42,43}$ The energy redistribution imprinted by electron-deficient heteroatoms in carbon materials has been widely reported by various groups; boron-doped graphene, for example, showed enhanced NRR performance $(10.8 \%$ faradaic efficiency at $-0.5 \mathrm{~V}$ ) due to the lower energy barrier for $\mathrm{N}_{2}$ electroreduction within $\mathrm{BC}_{3}$ structures. ${ }^{44}$ Furthermore, the presence of $\mathrm{BC}_{3}$ trimers within the hexagonal lattice of $\mathrm{C}$ materials induces a p-type semiconductor behavior and the resulting material displays unique sensing capabilities (e.g., to $\mathrm{NO}_{2}$ and $\mathrm{NH}_{3}$ toxic gases). ${ }^{45}$ Phosphorous incorporation by pyrolysis or hydrothermal treatment also results in materials with catalytic sites, which are widely utilized as supercapacitors and electrocatalysts. ${ }^{46-51}$ From the synthetic point of view, achieving homogeneity in the distribution of dopant atoms within the lattice is a standing challenge; our group developed an approach to produce homogeneous heteroatomdoped carbons by utilizing polycyclic aromatic hydrocarbons (PAHs) and heteroatom-containing species which melt during a thermal reaction. During the polycondensation of PAHs with

a)
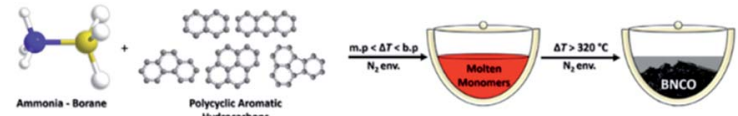

b)

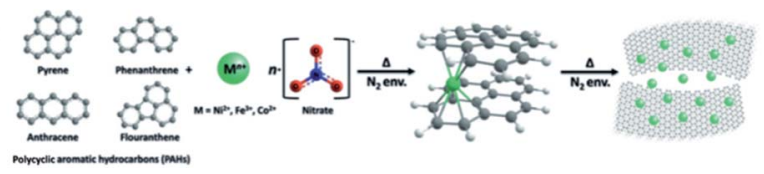

Fig. 1 Synthetic procedure schemes for (a) $\mathrm{C}-\mathrm{N}-\mathrm{B}-\mathrm{O}$ and (b) metalincorporated crystalline carbons using a molten-state synthesis. Reproduced with permission from ref. 52 and 53, copyright (2018) Wiley-VCH. 
heteroatom-containing molecules (or metal salts), their sublimation is quenched, and the structural carbon is completely preserved thanks to the formation of new covalent or coordination bonds. Using this method, boron, nitrogen, oxygen, or different metals have been successfully included within carbon materials and their performance has been tested in Li-ion batteries or an electrochemical oxygen evolution reaction (OER, see Fig. 1). ${ }^{52,53}$

\section{1. $\mathrm{N}$-Doped carbons, $\mathrm{C}_{2} \mathrm{~N}, \mathrm{C}_{3} \mathrm{~N}_{4}$ and other $\mathrm{C}-\mathrm{N}$ containing polymers}

Given nitrogen's electron-accepting ability and different atomic radius, its incorporation into a C-matrix alters the electronic structure and modifies the charge distribution (uneven across a $\mathrm{C}-\mathrm{N}$ bond) and the neighboring carbon atoms display a relatively positive charge that makes them more reactive in various catalytic scenarios. ${ }^{\mathbf{5 4 5 5}}$ Modulating the amount of nitrogen in this class of materials, ranging from N-doped carbons, $\mathrm{C}-\mathrm{N}$ based organic polymers, and $\mathrm{C}_{x} \mathrm{~N}_{y} \mathrm{H}_{z}$ derivatives, dramatically alters their electronic structure.

Carbon and nitrogen-based organic polymers allow a relatively easy design, functionality and processing, which make them suitable for a wide range of applications ranging from photo- and electro-catalysis to gas storage and more. ${ }^{56-62}$ Their porosity and the tunability of their chemical composition promoted their utilization as precursors for the synthesis of $\mathrm{N}$ doped carbon materials. ${ }^{63-65}$ Additionally, metal-organic frameworks (MOFs) and other highly ordered pre-organized molecular compounds have also been utilized for the hightemperature synthesis of heteroatom-modified carbon materials. ${ }^{66-70}$

Increasing the nitrogen amount in carbon materials can be done by using N-rich precursors; the thermal condensation of such molecules, e.g., barbituric acid, urea or triaminopyrimidine, amongst others, can imprint graphitic or pyridinic nitrogen sites into the structure and yield $\mathrm{C}_{3} \mathrm{~N}$ and $\mathrm{C}_{2} \mathrm{~N}$ structures with varying electronic and porous structures. ${ }^{71-73}$ Already

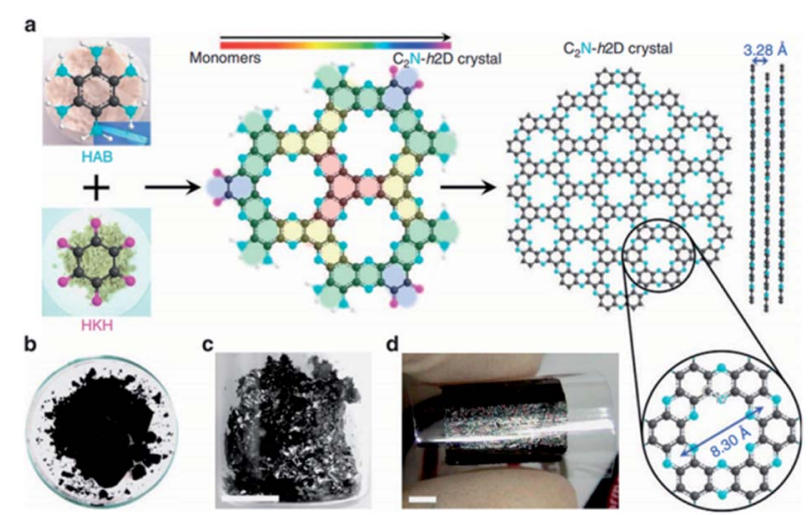

Fig. 2 Schematic representation of the synthesis of a $\mathrm{C}_{2} \mathrm{~N}$ material (a), and its corresponding digital images as prepared (b), solution cast over $\mathrm{SiO}_{2}$ after condensation at high temperatures (c) and in a film over a PET substrate (d). Reproduced with permission from ref. 75, copyright (2015) Nature publishing group. in 1986, Kouvetakis et al. have shown the preparation of $\mathrm{BC}_{3}$ and $\mathrm{C}_{5} \mathrm{~N}$ planar structures by thermal condensation of a monomer containing $\mathrm{B}$ and $\mathrm{N}$ at high temperatures (800-1400 $\left.{ }^{\circ} \mathrm{C}\right){ }^{74}$ Mahmood et al. reported for the first time the synthesis of a $\mathrm{C}_{2} \mathrm{~N}$ material suitable for field-effect transistors by a wetchemical reaction between hexaaminobenzene trihydrochloride and hexaketocyclohexane octahydrate in $\mathrm{N}$-methyl-2pyrrolidone (NMP) as shown in Fig. $2 .^{75}$

The same group later reported the synthesis of twodimensional (2D) polyaniline $\left(\mathrm{C}_{3} \mathrm{~N}\right)$ frameworks by the direct pyrolysis of hexaaminobenzene trihydrochloride single crystals. $^{76}$ Since these reports, several groups have thoroughly exploited these graphitic materials for various applications, such as photocatalysis or as anodes in Li-ion batteries. ${ }^{77-79}$ Nitrogen-rich frameworks $\left(\mathrm{C}_{3} \mathrm{~N}_{3}, \mathrm{C}_{3} \mathrm{~N}_{4}\right.$ or $\left.\mathrm{C}_{3} \mathrm{~N}_{5}\right)$ are more commonly exploited in photocatalytic scenarios given their typical semiconducting behavior that allows the harvesting of visible light with the concomitant generation of an electronhole $\left(\mathrm{e}^{-}-\mathrm{h}^{+}\right)$pair, which can reduce (electron) or oxidize (hole) molecules. ${ }^{\mathbf{8 0 , 8 1}} \mathrm{C}_{3} \mathrm{~N}_{3}$ materials are the less explored of this kind, and theoretical predictions have shown that a $\mathrm{C}_{3} \mathrm{~N}_{3}$ monolayer has a band gap $\left(E_{\mathrm{g}}\right)$ of $1.6 \mathrm{eV}$ and could potentially be used in devices for $\mathrm{H}_{2}$ separation from impurity gases. ${ }^{82}$ Experimentally, this stoichiometry has been reported by our group and others by either high pressure-high temperature treatments, which results in the inclusion of $\mathrm{P}$ atoms $\left(\mathrm{C}_{3} \mathrm{~N}_{3} \mathrm{P}\right)$ or by the thermal condensation of a supramolecular assembly containing triaminopyrimidine and cyanuric acid at low temperatures. ${ }^{\mathbf{8 3 , 8 4}}$ $\mathrm{C}_{3} \mathrm{~N}_{4}$ materials, commonly referred to as "carbon nitrides" or "melon" (first discovered by Liebig ${ }^{\mathbf{8 5}, 86}$ and typically labelled "CN"), have been the most widely studied and reported. The first $\mathrm{C}_{3} \mathrm{~N}_{4}$ structures reported were obtained through solvothermal means, ${ }^{\mathbf{8 7}}$ along with chemical and physical vapor deposition variants, which were summarized by Kroke et al. in a comprehensive review in $2004 .^{88}$ Despite the fact that $\mathrm{C}_{3} \mathrm{~N}_{4}$ is commonly referred to as a $2 \mathrm{D}$ analogue of graphene, the $\mathrm{C}_{3} \mathrm{~N}_{4}$ stoichiometry, composed of tri-s-triazine units with remaining $\mathrm{H}$ atoms at the boundaries (due to a non-ideal condensation), is structurally a diamond-like $\mathrm{CN}$ allotrope, a hard material reported to be harder than diamond. ${ }^{89-91}$

Increasing the $\mathrm{N}$-to-C ratio in carbon nitrides can result in novel properties, such as increased basicity; nevertheless, the synthesis of such materials involves the insertion of unstable $\mathrm{N}-\mathrm{N}$ bonds. Kumar et al. showed the synthesis of a novel $\mathrm{C}_{3} \mathrm{~N}_{5}$ material by the thermal deammoniation of melem hydrazine. The prepared framework exhibited a narrow $E_{\mathrm{g}}$ of $1.76 \mathrm{eV}$ and was evaluated in several applications ranging from photoelectrochemistry to dye adsorption. ${ }^{92}$ Additionally, Kim et al. recently prepared thermodynamically stable, mesoporous $\mathrm{C}_{3} \mathrm{~N}_{7}$ and $\mathrm{C}_{3} \mathrm{~N}_{6}$ by the thermal treatment of a tetrazole derivative at temperatures lower than $300{ }^{\circ} \mathrm{C}$. DFT calculations confirm the formation of $\mathrm{N}-\mathrm{N}$ bonds in the form of tetrazines and triazoles and the catalytic activity of the nitrogen sites was assessed in the ORR, which was enhanced using $\mathrm{C}_{3} \mathrm{~N}_{7} \cdot{ }^{93}$ Furthermore, the inclusion of $\mathrm{B}$ atoms has allowed the formation of semiconducting $\mathrm{C}_{9} \mathrm{~N}_{4}$ monolayers for an electrocatalytic NRR, as shown by Zhang et al. ${ }^{94}$ 


\section{Graphitic carbon nitrides: introductory aspects and applications}

Graphitic carbon nitride (a melon-based $\mathrm{C}_{3} \mathrm{~N}_{4}$, to which we refer here as $\mathrm{CN}$ ) is a $2 \mathrm{D}$ semiconductor based on $\mathrm{C}$ and $\mathrm{N}$ and residual hydrogen atoms, with remarkable chemical and electronic properties. ${ }^{95}$ It has been widely used in energy-related scenarios since the 2009 pioneering report that showed its ability to split water under visible light.96 The synthesis of this material entails the thermal treatment of $\mathrm{C}-\mathrm{N}$ monomers at high temperatures, which undergo a solid-state condensation reaction that releases ammonia and results in the formation of in-plane aromatic heptazine units with terminal amine groups, which interact with each other by van der Waals forces (Fig. 3). ${ }^{97-99}$

Many organic monomers containing carbon, nitrogen, and often heteroatoms like oxygen or sulfur have been used for the synthesis of $\mathrm{CN}$ such as: dicyanamide, ${ }^{100}$ melamine, ${ }^{\mathbf{1 0 1 - 1 0 5}}$ urea, ${ }^{106-109}$ thiourea, ${ }^{110-112}$ triazoles, ${ }^{113}$ and others, ${ }^{114-120}$ at different time intervals ${ }^{121}$ and temperatures ranging from 400 to $650{ }^{\circ} \mathrm{C} .{ }^{122-125}$ The utilization of different precursors yields $\mathrm{CN}$ materials with a wide range of properties and possible applications. ${ }^{126,127}$ To this end, we refer the reader to excellent previously published reviews for a wider picture on the synthesis and characterization of CNs using a single precursor. ${ }^{128-134}$ To date, $\mathrm{CN}$ has been applied mainly in photo(electro)catalytic reactions, ${ }^{135-139}$ with $\mathrm{H}_{2}$ production through water splitting, ${ }^{140-150} \mathrm{CO}_{2}$ reduction ${ }^{151-153}$ and degradation of organic pollutants ${ }^{154-156}$ being the most comprehensive and
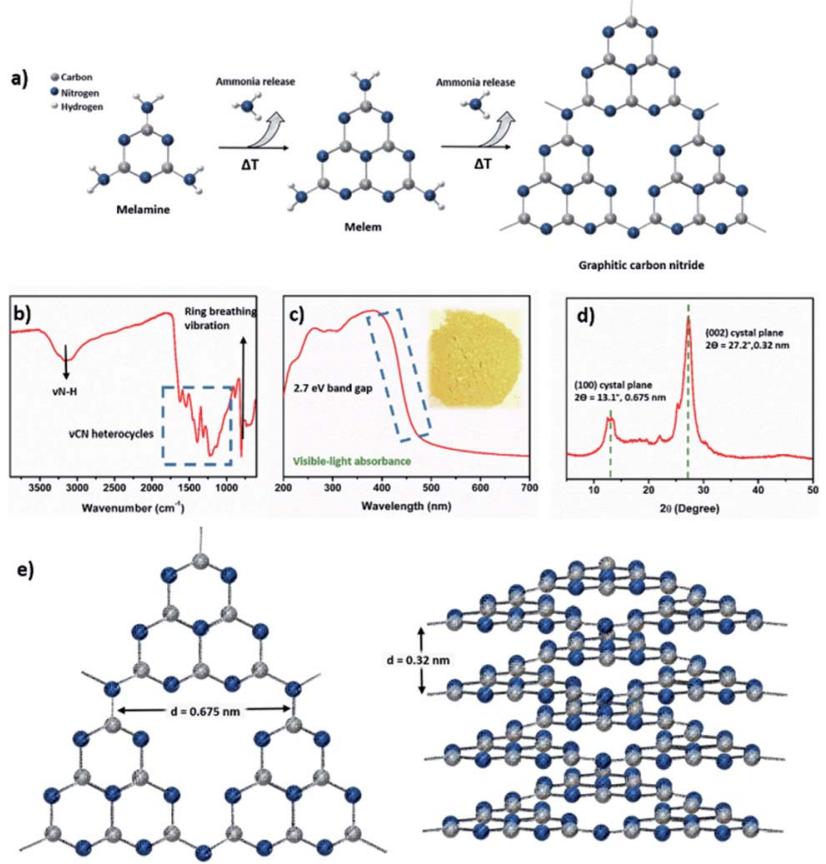

Fig. 3 (a) Polymerization pathway of melamine to CN. Characterization of $\mathrm{CN}$ powder by (b) Fourier-transform infrared spectroscopy (FTIR), (c) UV-vis absorption spectroscopy and (d) powder X-ray diffraction (XRD). (e) In plane and layered structure of $\mathrm{CN}$.

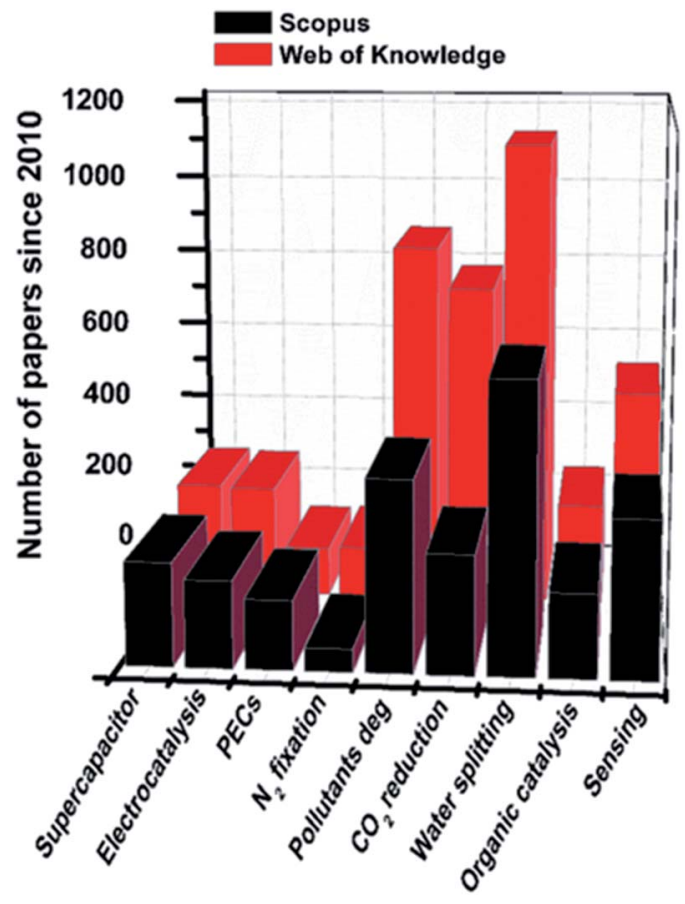

Fig. 4 Number of papers published in the last decade (since 2010) describing the use of CN materials for different applications. Data obtained from Scopus (www.scopus.com, Elsevier) and Web of Science (www.webofscience.com, Clarivate Analytics) on December $24^{\text {th }}, 2019$.

commonly reported. Nevertheless, the fine control over the electronic structure or morphology of CNs has allowed their exploitation in a wide scope of additional applications ${ }^{157-159}$ as illustrated in Fig. 4, including water oxidation, ${ }^{160-162}$ organic transformations, ${ }^{163-170}$ supercapacitors, ${ }^{171}$ biosensing, ${ }^{172-178}$ nitrogen fixation, ${ }^{179-186}$ emulsion stabilizers, ${ }^{187-189} \quad \mathrm{H}_{2} \mathrm{O}_{2}$ production, ${ }^{190}$ and even as micromotors. ${ }^{191}$

The solid-state reaction that solid monomers undergo during the $\mathrm{CN}$ synthesis, along with the required high temperatures and the poor solubility of the final product in common solvents, impedes a full control over their chemical and electronic properties, as well as their functionalization or implementation in devices. Therefore, achieving high performance in a given application with a realistic perspective of industrial application is not common. In the next two sections, we review the state of the art of the synthetic pathways to control the nanostructure and/or the chemical composition of $\mathrm{CN}$, via either a direct synthetic pathway or coupled to a post-synthetic treatment.

\section{Synthetic pathways for the control of $\mathrm{CN}$ properties}

During the last decade, different synthetic approaches have emerged in order to modify CN's properties towards an enhanced performance in photocatalytic scenarios. Improving optical absorption by tailoring energy band positions, achieving a high number of active sites through morphology and porosity 
control, and prolonging the lifetime of the photogenerated charge carriers have been the aims of the various demonstrated synthetic pathways. However, controlling these parameters is very challenging in a solid-state reaction and despite the recent progress, a complete and rational design of efficient photocatalytic CN systems from the reactant level is still a standing challenge.

\subsection{Copolymerization}

Copolymerization is one of the most common synthetic approaches to the preparation of $\mathrm{CN}$ materials with extended light absorption due to its simplicity. In this technique, a mixture of two or more small organic monomers, obtained by grinding, ball-milling or dissolution in common solvents, reacts at high temperature typically through Schiff-base reactions ${ }^{192}$ or nucleophilic attacks. Zhang et al. reported in 2010 the first $\mathrm{CN}$ material prepared by copolymerization, where 2-cyanoguanidine (dicyandiamide, DCDA) and various amounts of barbituric acid (BA) were dissolved in water before a thermal treatment at $550{ }^{\circ} \mathrm{C}$ (Fig. 5a). ${ }^{193}$ In this work the $\mathrm{C} / \mathrm{N}$ ratio increased up to 0.96 due to the replacement of nitrogen atoms in the melon ring by carbon, which entailed the narrowing of the CN's band gap to $1.58 \mathrm{eV}$ (from the 'pristine' $E_{\mathrm{g}}$ of $\mathrm{CN}, c a .2 .7 \mathrm{eV}$ ) and a substantial enhancement of $\mathrm{H}_{2}$ production through water splitting in a water-triethanolamine (TEOA) solution with Pt serving as a cocatalyst. Since this pioneering work, CN prepared by copolymerization of DCDA and BA has been widely used in different scenarios such as glutathione detection through photoactivated oxidase mimetics, ${ }^{194}$ degradation of aniline ${ }^{195}$ or detection of $\mathrm{Cu}^{2+} \cdot{ }^{196}$ The modification of the $\pi$-conjugated system of $\mathrm{CN}$ was studied by Zhang et al., who copolymerized DCDA with a wide library of different organic monomers containing an aromatic

a)

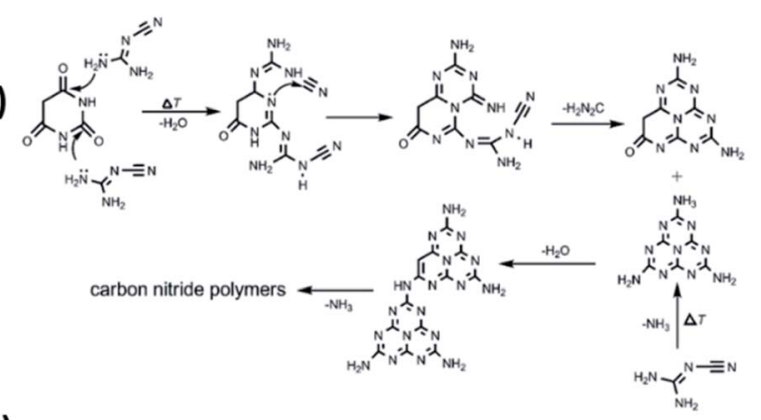

b)

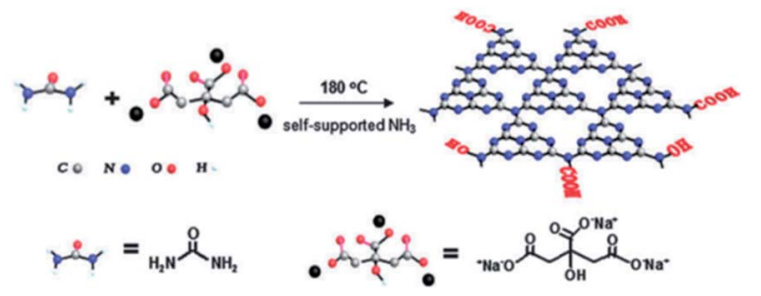

Fig. 5 (a) Copolymerization reaction between dicyanamide and barbituric acid. Reproduced with permission from ref. 193, copyright (2010) Wiley-VCH. (b) Formation of fluorescent CN quantum dots by a copolymerization reaction of urea and citric acid. Reproduced with permission from ref. 202, copyright (2013) Royal Society of Chemistry. ring. ${ }^{197}$ The highest photocatalytic performance for the hydrogen evolution reaction (HER) was obtained with a 5\% loading of 2-aminobenzonitrile $\left(147 \mu \mathrm{mol} \mathrm{h}^{-1}\right)$; the presence of aromatic carbon species in the $\mathrm{CN}$ structure was confirmed by ${ }^{13} \mathrm{C}$-solid-state nuclear magnetic resonance (NMR), which showed new peaks at 115 and 126 ppm.

Generally, a wide variety of monomers have been utilized for copolymerization with DCDA, which is the most common $\mathrm{C}-\mathrm{N}$ building block in this type of synthesis. ${ }^{198}$ Thiourea, for example, can decompose into gas bubbles during calcination, thereby inducing nanoporosity when copolymerized with DCDA and resulting in a 3.4 fold enhancement of the specific surface area $\left(S_{\mathrm{A}}\right)$, with an average pore size of $3.7 \mathrm{~nm}$, and an enhanced photocatalytic activity in the photodegradation of methylene blue (MB) dye. ${ }^{199}$ A decrease in the $E_{\mathrm{g}}$ of $\mathrm{CN}$ from 2.75 to 2.58 and $2.54 \mathrm{eV}$ was achieved by copolymerizing DCDA with uracil and diaminopyrimidine, respectively. These molecules replace some of the nitrogen with carbon and allow additional finetuning of the electronic structure and morphology towards enhanced $\mathrm{e}^{-}-\mathrm{h}^{+}$separation and photocatalytic performance in different model reactions. ${ }^{200,201}$ Despite the wider use of DCDA as the main $\mathrm{C}-\mathrm{N}$ building block for copolymerization, other precursors are also common. The high solubility and reactivity of urea allow the formation of fluorescent $\mathrm{CN}$ quantum dots (CNQDs) by copolymerization under hydrothermal conditions in the presence of sodium citrate (molar ratio $6: 1$, see Fig. $5 \mathrm{~b}$ ). The size of the obtained CNQDs ranges from 2.6 to $5.5 \mathrm{~nm}$, with thicknesses of 1.5-2.5 $\mathrm{nm}$ and the final material contains a high oxygen content, a consequence of the hydrothermal treatment and of the incorporation of oxygen from sodium citrate. Furthermore, the fluorescence quantum yield reached $42 \%$, resulting in a remarkable performance for HEK 293T cell imaging. ${ }^{202}$

A similar photoluminescent behavior was shown by introducing phenyl groups into $\mathrm{CN}$ through the copolymerization of urea with a small amount of trimesic acid. Song et al. observed that this treatment causes a downshift of the conduction band (CB) level, which results in a shift of the emission from blue (with two peaks at 443 and $457 \mathrm{~nm}$ ) to green (522 nm) upon excitation at $340 \mathrm{~nm}$. The fluorescence quantum yield of $14 \%$ allowed, for the first time, the application of $\mathrm{CN}$ for the imaging of latent fingerprints. ${ }^{203}$ Highly photoactive $\mathrm{CN}$ materials were also achieved by copolymerization of different precursor molecules with urea; ${ }^{204}$ aromatic moieties were induced by phenylurea, ${ }^{205}$ azoles, ${ }^{206,207}$ benzoic acid and others. ${ }^{208}$ These materials exhibited a narrower $E_{\mathrm{g}}$ and improved charge transfer and separation of photogenerated excitons towards improved performance in $\mathrm{H}_{2}$ production through water splitting.

Additionally, as mentioned previously, the copolymerization of urea with BA and other C-rich molecules can improve the optical absorption and charge separation by the creation of surface molecular heterojunctions that boost the HER and the $\mathrm{CO}_{2}$ photoreduction performance. ${ }^{209,210}$ A nanotube morphology with enhanced carrier mobility can also be obtained from mixtures of urea and oxamide; they display a similar structure and reactivity and therefore interact during thermal condensation to form a cross-linked complex, which upon further 
calcination yields a $\mathrm{CN}$ polymer with $1.3 \%$ AQY in the HER under green light illumination, ${ }^{\mathbf{2 1 1}}$ as a result of an enhancement in light harvesting and a red shift of the absorption edge up to $650 \mathrm{~nm} \cdot{ }^{212}$ Melamine is also widely applied in copolymerization due to its compatibility with a wide range of commercially available triazine-based molecules. In 2010, the formation of $\mathrm{N}$ rich (N/C ratio of 1.64) $\mathrm{CN}$ hollow vessels through the copolymerization of melamine and cyanuric chloride at a ratio of $2: 1$ was reported. ${ }^{213}$ In this case, the reaction took place in a sealed quartz tube at $800{ }^{\circ} \mathrm{C}$; the released gases during the condensation increase the inner pressure, which in turn contributes to the modification of the morphology and crystallinity of the final $\mathrm{CN}$ and leads to strong photoluminescence (PL) in the greenblue region.

Cyanuric chloride can be transformed into 2,4,6trihydrazino-1,3,5-triazine (THDT) by a reaction with hydrazine hydrate at $130{ }^{\circ} \mathrm{C}$ under reflux; the resulting monomer shows a high N/C ratio and its copolymerization with melamine alters the morphology and chemical composition of the final CN. The high nitrogen content, up to $77.2 \%$ as determined by X-ray photoelectron spectroscopy (XPS), disturbs the band structure of $\mathrm{CN}$, resulting in an $E_{\mathrm{g}}=1.98 \mathrm{eV}$ and a 2.3 -fold enhancement of the HER for a sample with a 0.15 mass ratio of THDT. ${ }^{214}$

Triaminopyrimidine, which contains one more $\mathrm{C}$ atom, successfully substitutes one nitrogen in the triazine ring of $\mathrm{CN}$ upon thermal condensation with melamine. This allows the narrowing of the $E_{\mathrm{g}}$ to $2.4 \mathrm{eV}$ by a negative shift in the valence band (VB), resulting in enhanced performance in NO removal. ${ }^{215}$ As shown, the choice of the precursor monomers to include in a copolymerization determines the microstructure, band structure and charge separation properties, thus affecting the overall performance in a given reaction.

Several groups have reported a higher photocatalytic activity for a carbon nitride derived from urea $v s$. a $\mathrm{CN}$ derived from other $\mathrm{C}-\mathrm{N}$ monomers, such as melamine, cyanamide or thiourea. ${ }^{\mathbf{1 2 6 , 2 1 6}}$ Similarly, Zhang et al. studied the copolymerization of common $\mathrm{C}-\mathrm{N}$ monomers (melamine, DCDA, thiourea and urea) with the amino acid glycine. Of all the copolymers formed, the one with urea showed the highest hydrogen evolution performance. ${ }^{217}$ Recently, the copolymerization of more than two C-N monomers has been described by Vidyasagar et al. ${ }^{218}$ In this work, phenylurea was copolymerized along with a melamine/urea mixture for a successful incorporation of phenyl groups. The assembly of phenyl units within the $\mathrm{CN}$ was confirmed by solid-state NMR, and the narrowing of the band gap contributes to a superior performance in $\mathrm{CO}_{2}$ reduction to formic acid and degradation of rhodamine $\mathrm{B}(\mathrm{RhB})$ dye. ${ }^{218}$

\subsection{Doping}

4.2.1. Metal doping. As shown before, a careful selection of the monomers taking part in the copolymerization process allows the formation of $\mathrm{CN}$ materials with tuned chemical compositions and tailored optical properties and/or electronic structures. Utilizing building blocks containing heteroatoms can lead to new $\mathrm{CN}$ materials with a modified chemical composition by the insertion of a given heteroatom (metal, halogen, chalcogen, pnictogen, etc.) within the $\mathrm{C}-\mathrm{N}$ matrix. The presence of foreign atoms has been proven to have beneficial effects on the photocatalytic performance, typically improving the charge transfer and separation of photogenerated excitons. ${ }^{219,220}$

The first evidence of the formation of a hybrid material through copolymerization was provided by Wang et al. in 2009, who reported the synthesis of a transition metal-modified $\mathrm{CN}$ by the copolymerization of DCDA with $\mathrm{FeCl}_{3}{ }^{221}$ The iron ions were included in the so-called "nitrogen pots" emulating the chemical structures of systems present in nature (e.g., chlorophyll, heme groups and coordination modes of phthalocyanines). The crystal structure of pristine $\mathrm{CN}$ is modified, with a significant shift in the in-plane period to $0.681 \mathrm{~nm}(v s .0 .713 \mathrm{~nm})$, and the coordination of iron to the nitrogen atoms in $\mathrm{CN}$ is confirmed by the absence of diffraction peaks corresponding to iron species. The prepared materials show enhanced optical absorption, confirming the modification of the electronic structure, which resulted in the enhancement of the photocatalytic activity towards RhB dye degradation. Since then different metal-modified CN materials have been shown. For example, Ding et al. extended this study to other transition metal cations, such as $\mathrm{Cu}^{2+}, \mathrm{Mn}^{2+}, \mathrm{Ni}^{2+}$ and $\mathrm{Co}^{3+}$. In this work, XPS characterization shows a systematic shift to lower binding energy of the $\mathrm{N} 1 \mathrm{~s}$ species in $\mathrm{C}=\mathrm{N}-\mathrm{C}$ units upon coordination with the metal cation, as well as the presence of all the metallic elements.

Furthermore, the improved charge separation properties, proven by PL measurements, resulted in an enhanced photocatalytic performance in the oxidation of benzene to phenol for all hybrid materials. ${ }^{222}$ Iron doping was evaluated with different $\mathrm{C}-\mathrm{N}$ building blocks as well, such as DCDA, glucose formamide and citric acid, to achieve iron and carbon doping. ${ }^{223,224}$ In both cases, the improved electron transfer resulted in an enhanced photocatalytic performance, for RhB degradation and the HER, respectively. Additionally, $\mathrm{Hu}$ et al. demonstrated how Fe-doped $\mathrm{CN}$, synthetized from the pyrolysis of melamine and $\mathrm{Fe}\left(\mathrm{NO}_{3}\right)_{3}$ $\cdot 9 \mathrm{H}_{2} \mathrm{O}$, is capable of performing $\mathrm{N}_{2}$ photofixation thanks to the activation of $\mathrm{N}_{2}$ molecules by $\mathrm{Fe}-\mathrm{N}$ bonds. ${ }^{225}$ These examples show that doping with transition metals gives access to a wide array of materials with improved photocatalytic activity. ${ }^{226}$ Copper, nickel, manganese or cobalt doping can be easily achieved by polymerization of a $\mathrm{C}-\mathrm{N}$ building block, namely DCDA, urea and others, ${ }^{227}$ with the corresponding transition metal chlorides $^{228-236}$ or nitrates. ${ }^{237}$ The use of vanadate salts has also been reported. ${ }^{238}$ Furthermore, the doping amount used in the synthesis can promote the coexistence of metal cations with different oxidation states, as shown recently by Dai et al., who prepared $\mathrm{CN}$ materials synergistically doped with $\mathrm{Cu}(\mathrm{I})$ and $\mathrm{Cu}(\mathrm{II}){ }^{239}$

Precious metals typically allow very high selectivity in organic reactions and, despite their high cost, they have also been incorporated as a dopant into $\mathrm{CN}$ lattices, thereby achieving improved photocatalytic performance. Eid et al. prepared homogeneous Pd-doped $\mathrm{CN}$ by the thermal polymerization of melamine with $\mathrm{K}_{2} \mathrm{PdCl}_{4}$ in the presence of nitric acid. The resulting $\mathrm{CN}$ nanowires show remarkable performance in 
the CO oxidation reaction thanks to the physicochemical properties of the nanowires and the catalytic activity of $\mathrm{Pd}$ atoms, achieving complete $\mathrm{CO}$ to $\mathrm{CO}_{2}$ conversion at $283^{\circ} \mathrm{C}$. The authors also demonstrated a synergistic effect between $\mathrm{Pd}$ and $\mathrm{Cu}$ atoms, which reduced the temperature needed for this oxidation down to $149{ }^{\circ} \mathrm{C}^{\mathbf{2 4 0}}$ Furthermore, $\mathrm{Pd} / \mathrm{Cu}$-doped $\mathrm{CN}$ showed enhanced electrocatalytic performance for $\mathrm{CO}_{2}$ reduction due to the quick charge transfer in the material, confirmed by impedance spectroscopy. ${ }^{241}$ Similarly, Au-Pd co-doped CN nanofibers were synthesized through copolymerization of melamine with $\mathrm{HAuCl}_{4}$ and $\mathrm{K}_{2} \mathrm{PdCl}_{4},{ }^{242,243}$ and Pt-Pd with $\mathrm{Na}_{2}$ $\mathrm{PtCl}_{4}$ and the same palladium salt. ${ }^{244}$ In all cases, the oxidation of CO was substantially enhanced compared to non-doped $\mathrm{CN}$ fibers.

Choi et al. carried out the complexation of $\mathrm{Al}^{3+}$ ions with $\mathrm{N}$ and $\mathrm{O}$ ligands by a rationally designed in situ keto-enol cyclization of urea and aluminum acetylacetonate $\left(\mathrm{Al}(\mathrm{acac})_{3}\right)$. The inclusion of $\mathrm{Al}^{3+}$ ions, proven by XPS, significantly enhances light absorption; an $E_{\mathrm{g}}=2.71 \mathrm{eV}$ was observed and both the $\mathrm{CB}$ and VB shifted to more positive values. The overall AQY for the HER reached $6.2 \%$ under $420 \mathrm{~nm}$ light illumination. ${ }^{245}$ Molecular silver species have also been incorporated into CN polymers by the copolymerization of silver tricyanomethanide with cyanamide, resulting in an improvement of the optical absorption and an enhanced performance in the selective hydrogenation of alkynes. ${ }^{246}$

Rare-earth metals such as yttrium and lanthanides (e.g., Eu and $\mathrm{Ce}$ ) have also been included in $\mathrm{CN}$ materials through copolymerization, generally achieving enhanced optical absorption, improved charge separation properties and an overall high photocatalytic activity. ${ }^{247-250}$

Alkali metals are another class of widely utilized metals along with a broad variety of semiconductor materials or molecular catalysts for enhancing their photocatalytic performance. ${ }^{251-256}$ The first reports on the doping of $\mathrm{CN}$ with $\mathrm{Na}^{+}$and $\mathrm{K}^{+}$, through the copolymerization of DCDA with $\mathrm{NaOH}$ and $\mathrm{KOH}$ respectively, showed the feasibility of effectively tuning the band gap with increasing alkali metal doping levels (Fig. 6). ${ }^{257,258}$
Including these cations within $\mathrm{CN}$ lattices typically increases the interplanar $d$ spacing, which influences the $S_{\mathrm{A}}$ and electronic band structure (effectively narrowing the band gaps to 2.50-2.55 eV, depending on the doping amount). The coordination of either $\mathrm{Na}^{+}$or $\mathrm{K}^{+}$to the pyridinic nitrogens of the triazine lattice results in hybridization between the dopant orbitals and molecular orbitals of $\mathrm{CN}$, which strongly modifies the potential of the VB and $\mathrm{CB}$, as confirmed by the VB analysis through XPS. This strong alteration of the reduction and oxidation potential of $\mathrm{CN}$ materials allows an enhancement in their performance in the photocatalytic degradation of organic pollutants. Since the pioneer studies on alkali-metal doping and the complementary theoretical reports confirming a strong electron transfer from the alkali metal to the CN moiety, which narrows the highest occupied molecular orbital-lowest unoccupied molecular orbital (HOMO-LUMO) gap, ${ }^{259}$ several studies have been reported on this topic, with different precursor salts $^{260-262}$ and C-N building blocks, ${ }^{263}$ with applications in solar fuel production. ${ }^{264}$

Additionally, the mediation of $\mathrm{CN}$ synthesis by $\mathrm{K}$ ions can induce useful chemical modifications, such as the creation of cyano groups, probably as a result of the formation and preservation of stable potassium cyanide intermediates. ${ }^{265}$ The presence of cyano groups at the surface of a $\mathrm{CN}$ photocatalyst can substantially improve the photocatalytic activity (e.g., the degradation of organic pollutants, ${ }^{266} \mathrm{HER},{ }^{267}$ or photocatalytic NRR). ${ }^{268}$ Besides the more widespread utilization of $\mathrm{Na}^{+}$and $\mathrm{K}^{+}$, theoretical and experimental studies show that other cations, namely $\mathrm{Li}^{+}$or $\mathrm{Mg}^{+}$, also significantly alter CN's electronic structure, which has beneficial effects in photocatalytic scenarios. ${ }^{269,270}$

4.2.2. Non-metal doping. Given the metal-free nature of $\mathrm{CN}$, much effort has been made towards designing an all-metalfree efficient photocatalytic system. In particular, introducing non-metallic elements into a $\mathrm{CN}$ lattice has attracted widespread attention. Utilizing small organic monomers containing $\mathrm{B}, \mathrm{P}, \mathrm{S}$, and so forth during calcination can lead to a certain concentration of the foreign element. The covalently bound a)

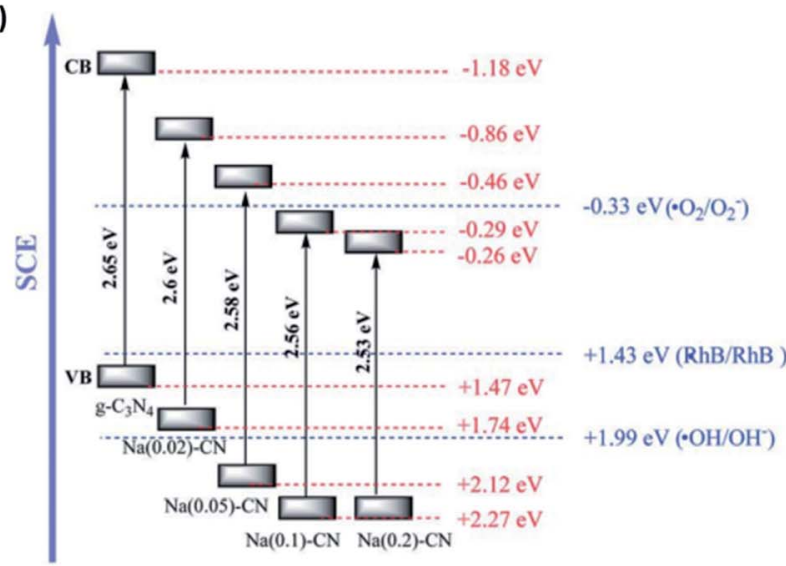

b)

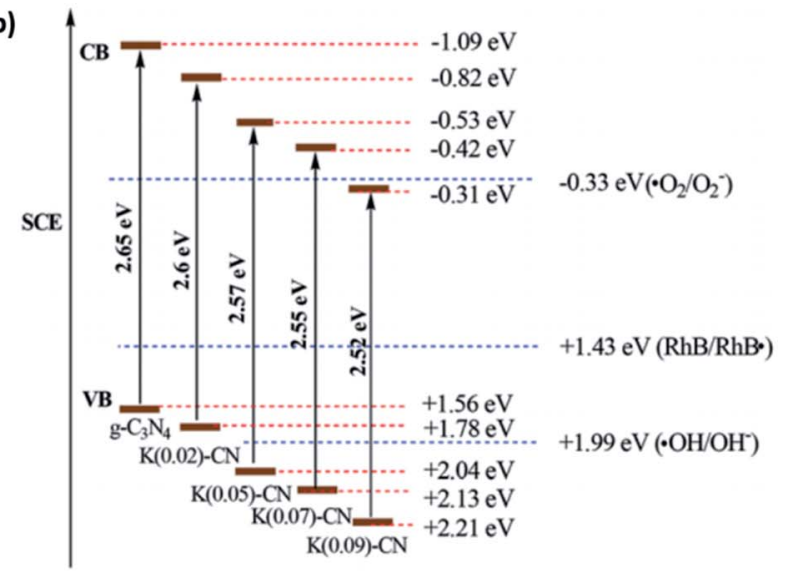

Fig. 6 Variation of the electronic structure of $\mathrm{CN}$ doped with (a) potassium and (b) sodium. Reproduced with permission from ref. 257 and 258 , copyright (2014, 2015 respectively) Royal Society of Chemistry. 
heteroatoms in $\mathrm{CN}$ materials strongly modify their electronic properties and improve the interaction with reactant molecules in catalytic scenarios. The first report on non-metal doping of $\mathrm{CN}$ through copolymerization was published by Yan et al. in 2010, where a solid mixture of melamine and boron oxide was heated to temperatures in the $500-600{ }^{\circ} \mathrm{C}$ range. The B-doped $\mathrm{CN}$, with boron atoms coordinated to tertiary amines, as proven by XPS, exhibits a blue shift in the optical absorption and quenching of the fluorescence. Both effects synergistically contribute to the measured enhancement in photocatalytic activity towards degradation of RhB and methyl orange (MO). ${ }^{271}$

Also in 2010, the fluorination of CN solids was carried out by Wang et al., who copolymerized DCDA and $\mathrm{NH}_{4} \mathrm{~F}$. Based on the strong electronegativity of fluoride ions, the authors hypothesized that $\mathrm{C}-\mathrm{F}$ bonds are formed, inducing a partial $\mathrm{C}-\mathrm{sp}^{3}$ character within the material, thus lowering the in-plane order of the material. Furthermore, the enlarged optical absorption corresponded to $E_{\mathrm{g}}=2.63 \mathrm{eV}$, and the photochemical properties were strongly modified; namely, the hydrogen production through water splitting was 2.7 times higher than that of bulk $\mathrm{CN}$, and a substantial improvement in benzene oxidation to phenol was observed. ${ }^{272}$ The same authors also reported codoping of $\mathrm{CN}$ with boron and fluorine as a result of the calcination of DCDA with the ionic liquid 1-butyl-3methylimidazolium tetrafluoroborate. ${ }^{273}$ These CNBF materials show excellent performance in the photooxidation reaction of cyclohexane due to their higher $S_{\mathrm{A}}\left(444 \mathrm{~m}^{2} \mathrm{~g}^{-1}\right.$, determined by nitrogen sorption measurements) coming from an increased interlayer distance $(d)$, lower $E_{\mathrm{g}}$, and enhanced charge separation properties derived from charge localization on the surface terminal sites.

Since these studies, doping of $\mathrm{CN}$ frameworks through copolymerization has become a bustling research field; the rational selection of heteroatom-containing organic monomers and different kinds of building blocks can result in the inclusion of non-metals such as B, P, S, O and halogens, which modify the selectivity in a given photocatalytic reaction and act as collection centers for $\mathrm{e}^{-}$or $\mathrm{h}^{+}$, enhancing the charge carriers' lifetime.

Boron is one of the most commonly utilized heteroatoms for incorporation into $\mathrm{CN}$ frameworks, due to its similar size to carbon and nitrogen, lower electronegativity, strong binding to nitrogen and influence on the final material's optical properties. These materials range from B-doped $\mathrm{CN}$ to BCN to C-doped boron nitride (BN). ${ }^{274}$ Wang et al. and others achieved the preparation of B-doped $\mathrm{CN}$ through the polymerization of DCDA and a borane ammonia complex; ${ }^{275}$ in this work, the $\mathrm{B}$ atoms were included mainly at the bay-carbon site, as proven by solid-state NMR and the enhanced catalytic activity of the material, which was tested for the oxidation of aliphatic $\mathrm{C}-\mathrm{H}$ bonds of several substrates. ${ }^{276}$ Sodium tetraphenylboron $\left(\left(\mathrm{C}_{6} \mathrm{H}_{5}\right)_{4} \mathrm{BNa}\right)$ has been utilized along with urea to achieve a fine control of the surface of the $\mathrm{CN}$ material. ${ }^{277}$ The phenyl leaving groups induce high $S_{\mathrm{A}}$ in the final CN (up to $144 \mathrm{~m}^{2} \mathrm{~g}^{-1}$ ), which promotes its facile delamination, reaching thin films of approximately 2-5 nm thickness. Furthermore, XPS proves the formation of $\mathrm{N}-\mathrm{B}-\mathrm{N}$ bonds, which strongly affects the band gap and photophysical properties of the materials. ${ }^{278}$ The prepared materials show an enhancement in the photocatalytic HER through water splitting with TEOA as a hole scavenger and Pt as a co-catalyst. Boric acid $\left(\mathrm{H}_{3} \mathrm{BO}_{3}\right)$ along with melamine, DCDA or thiourea has also been reported to achieve homogeneous $\mathrm{B}$ doping in $\mathrm{CN}$ frameworks and the altered electronic properties and morphology result in enhanced photocatalytic performances in the photodegradation of organic pollutants, ${ }^{279}$ photoreduction of uranium species $\left(\mathrm{UO}_{2}{ }^{2+}\right)^{280}$ and cycloaddition of $\mathrm{CO}_{2}$ to epoxide. ${ }^{281}$

Sulfur doping is complicated to achieve, due to the tendency of sulfur-containing moieties to leave (usually in the form of sulfates) upon calcination. Nevertheless, the use of monomers which contain sulfur for the synthesis of $\mathrm{CN}$ has been proven to successfully achieve its inclusion within the lattice. For this, trithiocyanuric acid and thiourea have typically been the monomers of choice since the first report describing their pyrolysis and transformation into S-doped $\mathrm{CN}$ for water oxidation and reduction, respectively. ${ }^{117,282}$ Indeed, various reports described the preparation of SCN materials with proven photocatalytic performance in water splitting, ${ }^{283}$ degradation of dyes and organic pollutants, ${ }^{284-286}$ oxygen reduction ${ }^{287}$ and $\mathrm{CO}_{2}$ reduction. ${ }^{288}$ SCN has also been applied in bioimaging applications; namely, its confinement through top-down techniques such as ultrasonication yields highly fluorescent S-doped CN QDs with redshifted emission, good biocompatibility and low toxicity, as shown in the imaging of PC-3 cells. ${ }^{289}$ Furthermore, the enhanced chemiluminescence of SCN vs. bulk-CN was shown by Zhu et al. The strong electrochemiluminescence signals of SCN can be quenched in the presence of $\mathrm{Cu}^{2+}$, which allows the quantification of L-cysteine. ${ }^{290}$ Selenium has also been incorporated into $\mathrm{CN}$ materials and they have been utilized in different scenarios. Qiao et al. showed the synthesis of Se-CN by copolymerization of DCDA, cyanuric acid (CA) and $\mathrm{SeO}_{2}$, which resulted in a se loading of 2.1 atomic \%. This material could oxidize tetramethylbenzidine (TMB) in the presence of $\mathrm{H}_{2} \mathrm{O}_{2} \cdot{ }^{291} \mathrm{Kumar}$ et al. followed the same synthetic route and utilized the prepared Se-CN for the efficient photocatalytic production of formaldehyde from $\mathrm{CO}_{2} \cdot{ }^{292}$

Phosphorus atoms within $\mathrm{CN}$ frameworks can strongly alter electrical or thermal conductivity, permitting their use in different applications, mainly relying on the enhanced charge mobility in the P-containing $\mathrm{CN}^{293}$ One of the first reports on the synthesis of PCN through copolymerization involved the thermal treatment of DCDA with the ionic liquid 1-butyl-3methylimidazolium hexafluorophosphate. ${ }^{294}$ Ionic liquids have been previously used for the introduction of dopants such as $\mathrm{B}$ or F, and in this case, EDS analysis determined that the presence of the phosphate salt in the initial reactant resulted in a weight percentage (wt $\%$ ) of $0.5 \% \mathrm{P}$ in the final material; the inclusion of $\mathrm{P}$ in the $\mathrm{CN}$ matrix was confirmed by XPS. The enhancement in photocatalytic performance induced by the extended optical absorption and the improvement in the charge separation properties was confirmed by visible-light degradation of RhB and MO. ${ }^{295}$ Since then, the most commonly utilized species for the synthesis of PCN through copolymerization have been phosphate salts ${ }^{277,296-298}$ or phosphonic acids, associated 
with common monomers such as DCDA or melamine. ${ }^{\mathbf{2 9 9 , 3 0 0}}$ In these scenarios, the photocatalytic activity towards the degradation of model organic dyes or a water-splitting HER was substantially enhanced due to the more beneficial electronic structure after $\mathrm{P}$ inclusion in $\mathrm{CN}$ frameworks.

Phosphonitrilic chloride trimer monomer (PCT) has also been utilized for the formation of co-polymers containing $\mathrm{C}-\mathrm{N}-\mathrm{P}$ elements, taking advantage of its high phosphorous content and a melting point $\left(112-115{ }^{\circ} \mathrm{C}\right)$ that allows the formation of a homogeneous medium upon thermal treatment. In 2014, Zhou et al. carried out the copolymerization of guanidinium hydrochloride as a CN precursor with PCT; the resulting material possessed $\mathrm{P}$ atoms, which replaced carbon in the $\mathrm{CN}$ lattice, as shown by XPS and ${ }^{31} \mathrm{P}$ solid-state NMR. As a result, the photocatalytic performance in hydrogen production and RhB degradation was substantially enhanced. ${ }^{301}$ The same monomer was copolymerized with thiourea and the resulting material was tested for photocatalytic NO removal..$^{302}$ Our group reported the thermal copolymerization of PCT with 2,4-diamino-6-phenyl1,3,5-triazine (DPT) and the formation of $\mathrm{C}-\mathrm{N}-\mathrm{P}$ materials with remarkable fire-resistance performance. ${ }^{303}$ In this case, both monomers create a liquid-state intermediate that allows an enhanced reactivity at higher temperature and the formation of CNP materials through ring opening of PCT and the nucleophilic attack of the amine group at the chlorine, before the final condensation takes place (Fig. 7). The inclusion of $\mathrm{P}$ in the $\mathrm{CN}$ framework reaches up to $32 \mathrm{wt} \%$, and the prepared materials show an impressive resistance to oxidation.

Another pnictogen (group 15), antimony (Sb), was included within $\mathrm{CN}$ frameworks by copolymerization of urea and sodium antimonate $\left(\mathrm{NaSbO}_{3}\right)$. The resulting $\mathrm{N}-\mathrm{Sb}$ (III) bonds were shown to perform as charge-capture centers, leading to a strong enhancement of the photocatalytic HER, with a $6.4 \%$ AQY at $420 \mathrm{~nm} .{ }^{304}$ Doping CN semiconductors with oxygen was predicted to improve visible-light harvesting through the improvement of charge carrier mobility. ${ }^{305}$ Qiu et al. achieved
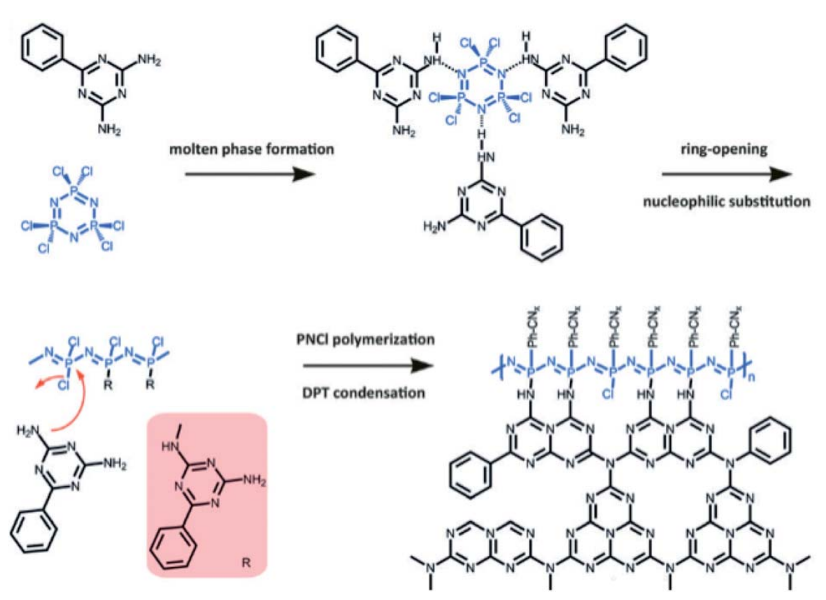

Fig. 7 Synthetic pathway for carbon-nitrogen-phosphorous (CNP) materials by copolymerization of 2,4-diamino-6-phenyl-1,3,5-triazine (DPT) and phosphonitrilic chloride trimer (PCT). Reproduced with permission from ref. 303, copyright (2018) Wiley-VCH. the extension of the optical absorption of $\mathrm{CN}$ up to $700 \mathrm{~nm}$ by inserting oxygen in its lattice, partially replacing nitrogen atoms. In this work, the doping with oxygen was achieved by copolymerization of urea with oxalic acid, and the resulting material showed an increased photocatalytic performance in the degradation of bisphenol A (BPA), with hydroxyl radicals playing the major role in the photocatalytic reaction. ${ }^{306}$ Another oxygen-rich monomer, citric acid, was copolymerized with thiourea in order to achieve simultaneous oxygen- and sulfurdoping in the final $\mathrm{CN}$ material. In this case, the oxygen doping was obtained by the partial retention of carboxylic acid groups after the hydrothermal reaction at $200{ }^{\circ} \mathrm{C}$; this strongly altered the PL properties, resulting in blue fluorescence with a $14.5 \%$ AQY and allowing a remarkable performance in the detection of mercury ions and cell imaging. ${ }^{307}$

Obtaining the inclusion of several heteroatoms that synergistically contribute to the modification of the electronic structure and improvement of the overall photocatalytic performance can be achieved by rationally selecting heteroatom-containing organic monomers and will take place at the elevated temperatures of the synthesis. Thiourea copolymerized with PCT incorporates both sulfur and phosphorous atoms in the CN lattice, which improves light harvesting and $S_{\mathrm{A}}$ compared to pristine or single-element-doped CN. ${ }^{308}$ Similarly, thiourea along with ionic liquids containing $\mathrm{PF}_{6}$ groups or phosphate salts, successfully yields S-P co-doped CN frameworks. ${ }^{309}$ Furthermore, DFT calculations performed by Liu et al. show that doping of $\mathrm{P}$ atoms preferentially occurs by replacing $\mathrm{C}$ atoms or occupying the interstitial sites of $\mathrm{CN}$, while $\mathrm{S}$ or $\mathrm{O}$ doping can replace $\mathrm{N}$ or be included in an interstitial site. ${ }^{310}$

The modification of CN layers with halogens has been predicted to occur more feasibly in the interstitial position; their introduction has been proven to lead to new density states and redistribution of the HOMO and LUMO energy levels. Fluoride perturbs the VB and HOMO due to its high electronegativity; meanwhile, chloride, bromide and iodide are involved in the conduction band and LUMO. Overall, the calculations performed by Zhu et al. illustrated that halogen-doped $\mathrm{CN}$ possesses a narrower $E_{\mathrm{g}}$, increased light absorption and reduced work function. ${ }^{311}$ Experimentally, after the first report by Wang et al. ${ }^{272}$ describing the fluorination of $\mathrm{CN}$ polymers, Zhang et al. extended this study by carrying out the copolymerization of DCDA with various ammonium halide salts $\left(\mathrm{NH}_{4} \mathrm{X}, \mathrm{X}=\mathrm{F}, \mathrm{Cl}, \mathrm{Br}\right.$ and $\left.\mathrm{I}\right)$ and testing the resulting halogenmodified $\mathrm{CN}$ materials as photocatalysts for the HER; CN-I showed the highest performance, reaching an AQY of $2.4 \%$ at $420 \mathrm{~nm} .{ }^{312}$ The extension of the aromatic CN system thanks to halogen insertion results in an enhanced optical absorption; the band gap narrowing was proven by VB analysis through XPS (supported by DFT calculations). This finding can be explained by the delocalization of electrons that interact with the $\pi$-electron system of CN. Further evidence of the photocatalytic enhancement of $\mathrm{CN}$ by iodine doping was provided by Guo et al., who achieved simultaneous $\mathrm{K}$ and $\mathrm{I}$ inclusion into $\mathrm{CN}$ frameworks by copolymerization of DCDA and KI. ${ }^{313}$ Additionally, Lan et al. studied the effect of the $\mathrm{C}-\mathrm{N}$ precursor in the synthesis of Br-modified $\mathrm{CN}$ materials. Urea, DCDA, $\mathrm{NH}_{4} \mathrm{SCN}$ 
and thiourea were chosen for the copolymerization along with $\mathrm{NH}_{4} \mathrm{Br}^{314}$ As predicted by others, ${ }^{126,216} \mathrm{Br}-\mathrm{CN}$ prepared with urea resulted in higher $\mathrm{H}_{2}$ evolution rates and photocatalytic OER with Pt and cobalt oxide as co-catalysts, respectively.

In addition to the popular method of $\mathrm{CN}$ doping with heteroatoms through direct copolymerization, it is also possible to include foreign elements within $\mathrm{CN}$ matrices in a postsynthetic manner, which can successfully alter the electronic structure and enhance the photocatalytic performance of $\mathrm{CN}$ derived materials. ${ }^{219,220,315}$

Lui et al. showed the incorporation of sulfur atoms by the thermal treatment of pre-formed $\mathrm{CN}$ under a $\mathrm{H}_{2} \mathrm{~S}$ atmosphere and observed an enhancement of photocatalytic activity in several model reactions including the HER, phenol degradation and the photogeneration of hydroxyl radicals. The sulfur atoms are incorporated by replacing lattice nitrogen atoms. Since new $\mathrm{C}-\mathrm{S}$ bonds are formed, the band gap is enlarged by the widening of the modified CN's VB stemming from the interaction of S 3p states with $\mathrm{N} 2 \mathrm{p}$ states. ${ }^{316}$ Another a chalcogen, i.e., Se, was introduced by the thermal treatment of a mixture consisting of $\mathrm{CN}$ and diphenyl selenide in ethanol, which resulted in the exfoliation of the product into thin nanosheets with thicknesses in the range of $0.7-2 \mathrm{~nm}$. The Se atoms replace heptazine nitrogens and strongly modify the electronic structure towards enhanced photocatalytic performance in the $\mathrm{CO}_{2}$ reduction and HER. ${ }^{317}$ Other non-metals have been inserted within $\mathrm{CN}$ using similar approaches, for example, boron in the form of borate. The treatment of $\mathrm{CN}$ with $\mathrm{NaBH}_{4}$ in water results in the formation of $\mathrm{Na}_{3} \mathrm{BO}_{3}, \mathrm{H}_{3} \mathrm{BO}_{3}$ and hydrogen, which is a strong reducing agent that attacks $\mathrm{N}$ atoms and creates nitrogen defects, allowing the insertion of $\mathrm{BO}_{3}{ }^{-}$species. The reductive treatment results in an $S_{\mathrm{A}}$ enhancement and in a narrowed band gap down from 2.71 to $2.66 \mathrm{eV}$, which contributes synergistically to the enhanced photocatalytic activity tested for NO removal. ${ }^{318}$ Phosphorous doping was achieved by wet grinding a urea-derived $\mathrm{CN}$ along with $\mathrm{NaH}_{2} \mathrm{PO}_{2}$, followed by thermal treatment at $400{ }^{\circ} \mathrm{C}$. The introduced $\mathrm{P}$ atoms replace corner-site $\mathrm{C}$ atoms, which results in $E_{\mathrm{g}}=2.52 \mathrm{eV}$ with a negative shift of both the $\mathrm{CB}$ and VB. The enhanced $S_{\mathrm{A}}$ and hydrophilicity contribute as well to an improved HER performance (AQY of $6.52 \%$ at $420 \mathrm{~nm}){ }^{319}$

The introduction of metals by a simple treatment of $\mathrm{CN}$ with metallic species has also been demonstrated. Typically, $\mathrm{N}$ atoms from $\mathrm{CN}$, which serve as Lewis bases, coordinate to the utilized metal (cation) Lewis acid. Doping with alkaline metals, such as potassium or cesium, by the thermal treatment of a mixture containing prepared $\mathrm{CN}$ with either $\mathrm{KCl}$ or $\mathrm{CsCl}$ was recently reported. ${ }^{320,321} \mathrm{Cu}^{2+}$ has also been widely inserted into $\mathrm{CN}$ matrices in a post-synthetic manner; Ju et al. showed that $\mathrm{Cu}-$ modified $\mathrm{CN}$, prepared by a reaction between urea-derived $\mathrm{CN}$ and $\mathrm{CuCl}_{2}$, exhibits a better separation of the HOMO and LUMO distributions, which in turn leads to an enhanced production of reactive oxygen species (ROS) upon visible-light illumination. ${ }^{322}$ Furthermore, Vázquez-González et al. followed the same synthetic approach and demonstrated that $\mathrm{Cu}-\mathrm{CN}$ can act as a heterogeneous catalyst mimicking the function of horseradish peroxidase. ${ }^{323}$ Other cations (e.g., $\mathrm{Ru}^{3+}$ and $\mathrm{Zn}^{2+}$ ) were incorporated following similar procedures and their photocatalytic performances were tested for organic catalysis and the HER. ${ }^{324,325}$ Lanthanum was also loaded by hydrothermally treating $\mathrm{CN}$ nanosheets with $\mathrm{La}\left(\mathrm{NO}_{3}\right)_{3}$; the resulting material showed strong fluorescence and outstanding performance in the detection of $\mathrm{Fe}^{3+}$ ions. ${ }^{326}$ Doping with halogens, such as iodine, was described by Han et al., who ball-milled melaminederived $\mathrm{CN}$ with iodine in different ratios and observed, using XPS, that the formed C-I bonds could narrow the $E_{\mathrm{g}}$ down to $2.37 \mathrm{eV}$ and enhance the photocatalytic HER, reaching an AQY of $3 \%$ at $420 \mathrm{~nm}^{327}$

\subsection{Templating}

4.3.1. Hard templating. One of the parameters that determines the overall photocatalytic performance of a given reaction is the number of active sites on the catalyst's surface. Tailoring the morphology and the porosity of $\mathrm{CN}$ materials is therefore an important strategy to overcome the bottlenecks towards high yields in photocatalytic reactions. The utilization of templates has permitted achieving significant progress in the controlled synthesis of $\mathrm{CN}$ nanostructures with a defined morphology, porosity and particle size distribution. This synthetic approach involves filling or coating a rigid template (typically made of inorganic materials as $\mathrm{SiO}_{2}, \mathrm{Al}_{2} \mathrm{O}_{3}$, etc.) with a $\mathrm{C}-\mathrm{N}$ precursor, thermal condensation at high temperatures into a polymeric $\mathrm{CN}$ structure and finally, etching the template (commonly using an acidic wash, Fig. 8)..$^{328,329}$

Already in 2005, Groenewolt et al. carried out the synthesis of CN nanoparticles (NPs) in different solid silica matrices with pore sizes in the $5-70 \mathrm{~nm}$ range. ${ }^{330}$ The porous templates were filled with cyanamide (CY) and the nanosized $\mathrm{CN}$ material was obtained via a thermal treatment at $550^{\circ} \mathrm{C}$. The first evidence of an enhancement in the catalytic performance by templated $\mathrm{CN}$ was given later in 2006 by Goettmann et al., who utilized $\mathrm{SiO}_{2}$ NPs of $12 \mathrm{~nm}$ for the synthesis of mesoporous CN, using CY as a molecular precursor. Electron microscopy along with the analysis of nitrogen sorption using the Brunauer-EmmettTeller (BET) model confirmed weak microporosity and enhancement of $S_{\mathrm{A}}$ up to $439 \mathrm{~m}^{2} \mathrm{~g}^{-1}$, which resulted in improved activity for the Friedel-Crafts acylation of benzene with hexanoyl chloride, reaching $90 \%$ conversion and a TOF of $6200 \mathrm{~h}^{-1}{ }^{331}$ Vinu et al. used two different precursors, namely

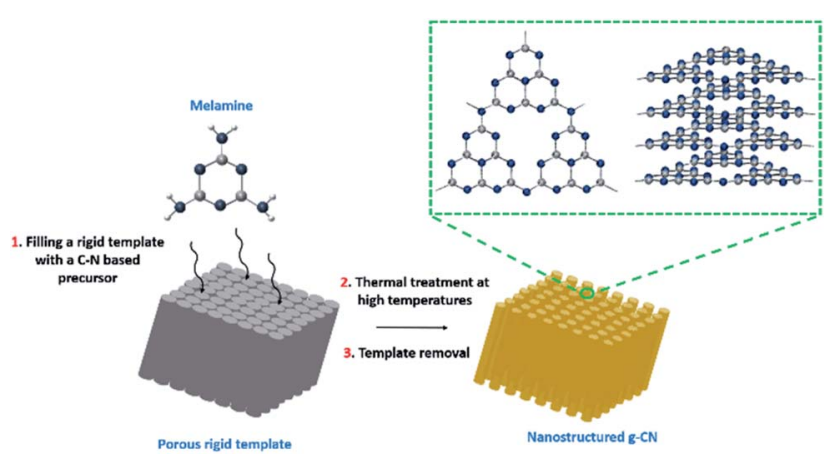

Fig. 8 A generic synthetic pathway for templated $\mathrm{CN}$. 
ethylenediamine (EDA) and $\mathrm{CCl}_{4}$ along with SBA-15 (a family of mesoporous silica templates with different pore diameters), ${ }^{332}$ for the synthesis of well-ordered materials with a pore diameter range of 4.2-6.4 nm. ${ }^{333}$ The catalytic activity was tested in Friedel-Crafts acylation of benzene using hexanoyl chloride and the prepared materials show a $100 \%$ conversion to caprophenone thanks to their high $S_{\mathrm{A}}$, reaching $830 \mathrm{~m}^{2} \mathrm{~g}^{-1} \cdot{ }^{333}$ EDA and $\mathrm{CCl}_{4}$ were further utilized for the synthesis of $\mathrm{CN}$ nanotubes templated by porous anodic aluminum oxide (AAO) and as well for preparing mesoporous nanoparticles (>150 nm size) templated with ultra-small silica nanoparticles. After the formation of the nanotubes at high temperature and the removal of the AAO template by treatment with $\mathrm{NaOH}$, the prepared materials were utilized as a nanotubular support for the deposition of $\mathrm{Pt}$ nanoparticles; this hybrid nanostructure was used for the hydrogenation of cyclohexene to cyclohexane. ${ }^{334}$ Furthermore, the high nitrogen content in the prepared $\mathrm{CN}$ nanoparticles gives rise to numerous basic sites in the form of amine or imine groups that promote the trans-esterification of $\beta$-keto esters, in synergy with the enhanced $S_{\mathrm{A}}\left(645 \mathrm{~m}^{2} \mathrm{~g}^{-1}\right) .{ }^{335}$ Confined nanorods of a $260 \mathrm{~nm}$ diameter with directional charge transport properties were synthesized utilizing an AAO template and $\mathrm{CY}$

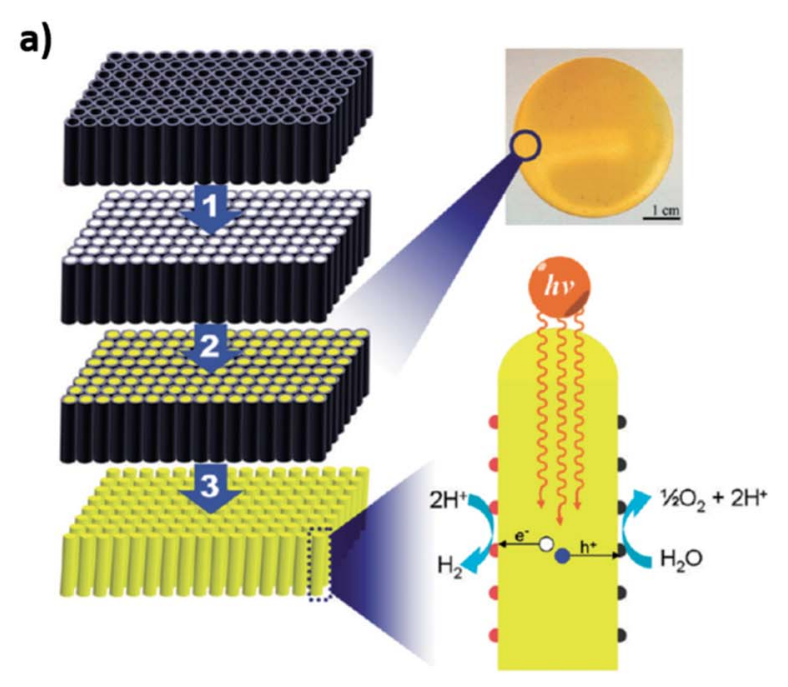

b)

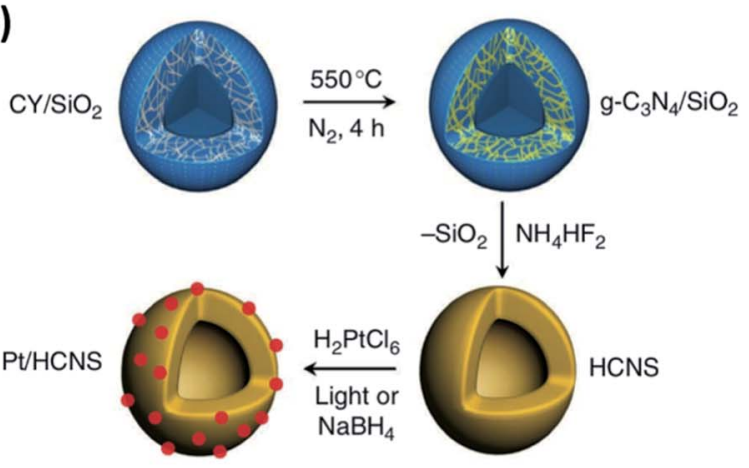

Fig. 9 (a) Synthesis of $\mathrm{CN}$ nanorods confined in an AAO template. Reproduced from ref. 336. Copyright (2011) American Chemical Society. (b) Synthesis of CN nanospheres utilizing silica nanoparticles. Reproduced with permission from ref. 338, copyright (2012) Nature publishing group. as a $\mathrm{C}-\mathrm{N}$ precursor; the confinement inside the nanochannels of the template enhances the orientation and crystallinity of the resulting $\mathrm{CN}$ with a lower HOMO given the lack of both free $\mathrm{NH}_{2}$ groups and defects within the structure (Fig. 9a). The photoactivity of the nanorods was substantially enhanced compared to bulk $\mathrm{CN}$ in the $\mathrm{H}_{2}$ and $\mathrm{O}_{2}$ evolution reactions, ${ }^{336}$ as was also confirmed by others. ${ }^{337}$ Further insight into HER performance enhancement using templating was provided by Sun et al. who used hollow silica nanoparticles along with CY for the formation of CN nanospheres, which after the template removal and light-induced deposition of Pt as a co-catalyst, showed a high HER AQY of $7.5 \%$ at $420.5 \mathrm{~nm}$. This fact is attributed mainly to the maximized light harvesting due to inner reflections and photonic effects within the nanostructure of the $\mathrm{CN}$ vesicle (Fig. 9b). ${ }^{338} \mathrm{Si}$ and co-workers reported a similar strategy, using silica spheres as nanoreactors to achieve the confined thermal polymerization of cyanamide precursors under pressurized conditions. The resulting nanosized $\mathrm{CN}$ showed a nanoporous structure as well as improved crystallinity and yielded a 44- and 30-fold HER enhancement under visible-light irradiation compared to bulk $\mathrm{CN}$ and a material prepared under atmospheric pressure, respectively. ${ }^{339}$

$\mathrm{SiO}_{2}$ nanoparticles have also been utilized to alter the electronic structure of $\mathrm{CN}$ forming nanojunctions and improve the photocatalytic performance in the degradation of RhB. Shalom et al. demonstrated that the polymerization of a $\mathrm{DCDA} / \mathrm{SiO}_{2}$ mixture yields a $\mathrm{CN} / \mathrm{SiO}_{2}$ hybrid composite, which exhibits improved charge separation efficiency upon illumination besides enhanced $S_{\mathrm{A}}{ }^{340}$ In the same way, aluminum oxide $\left(\mathrm{Al}_{2} \mathrm{O}_{3}\right)$ loaded with nickel was utilized to generate $\mathrm{CN}$ through the polymerization of EDA and $\mathrm{CCl}_{4}$ resulting in Ni NPs covered with 3-4 layers (calculated) of $\mathrm{CN}$. The $\mathrm{CN} / \mathrm{Ni} / \mathrm{Al}_{2} \mathrm{O}_{3}$ composites were used for the hydrogenation of $p$-nitrobenzoic acid at different $\mathrm{pH}$ values, where they showed remarkable conversion for more than 20 cycles. This performance stems from the donation of electrons from $\mathrm{Ni}$ to $\mathrm{CN}$, which enhances its capability to activate hydrogen for its reaction with nitro compounds. ${ }^{341}$ Despite the variety in the utilized templates in this synthetic approach, ${ }^{342-345}$ in general, SBA-15 has been the most commonly used hard material to tailor the nanostructure of $\mathrm{CN}$ polymers. Carrying out the polymerization at different temperatures, ${ }^{346}$ modifying SBA-15 by cross-linking or protonation $^{347,348}$ and testing the photocatalytic performance in different model reactions ${ }^{349}$ have been some of the recent developments in this research line. Furthermore, SBA-15 as well as common silica NPs allow the combination of this synthetic pathway with other modifications such as doping or copolymerization, by simple manipulation of the initial reactants (cast onto a hard template).

Utilizing thiourea to achieve S-doping ${ }^{282,350}$ or common $\mathrm{C}-\mathrm{N}$ monomers along with metal salts $\left(\mathrm{Fe}^{2+},{ }^{351} \mathrm{Cu}^{2+},{ }^{352,353}\right.$ and others ${ }^{354,355}$ ) together with templating agents permitted combining the electronic modification of $\mathrm{CN}$ towards an improved charge transfer and/or separation with a precise engineering of the morphology and porosity, with the aim of improving the photocatalytic activity in several model reactions. Additionally, Chen et al. carried out the copolymerization of 
DCDA with thiophene within a silica NP template to create mesopores in a $\mathrm{CN}$ framework that included conjugated functional groups, thus achieving synergistic molecular and textural engineering of the prepared material towards an efficient photocatalyst for the selective oxidation of alcohols. ${ }^{356}$

4.3.2. Soft templating. Soft templating slightly differs from hard templating; in this approach, molecular building blocks, namely, surfactants, ionic liquids or block polymers, are utilized in order to direct the synthesis of CN. A self-assembly process between the CN-polymers' reactants and the molecular templating agents imprints a given morphology during the calcination process. The templating molecules typically decompose before reaching the final synthesis temperature, which avoids the use of strong acids or basic agents for their removal from the product, as in the case of hard templating. ${ }^{328}$ The first evidence of the effectiveness of this technique was provided in 2010 by Wang et al., who used a wide variety of nonionic surfactants, block copolymers and ionic liquids (ILs) along with DCDA for the synthesis of mesoporous CN. Here, instead of the typical thermal treatment at high temperatures, intermediate sequences were utilized at lower temperatures before reaching the final condensation step at $550{ }^{\circ} \mathrm{C}$ to allow a structural rearrangement to occur before the decomposition of the soft template.

Parameters such as the crystal structure and elemental composition are strongly altered by the type of template and its relative amount; for example, when using Pluronic P123, the molar $\mathrm{C} / \mathrm{N}$ ratio varies from 0.82 to 2.06 due to the insertion of carbons from Pluronic P123 into the structure of the final CN. Ionic surfactants as cetyltrimethylammonium chloride and cetyltrimethylammonium bromide (CTA) lead to $S_{\mathrm{A}}=80 \mathrm{~m}^{2}$ $\mathrm{g}^{-1}$, while Triton X-100 provides high organization of the pore walls, with pore sizes ranging from 3.8 to $15 \mathrm{~nm}$ and $S_{\mathrm{A}}=76 \mathrm{~m}^{2}$ $\mathrm{g}^{-1}$. Furthermore, utilizing ionic liquids as templates proved to induce nanoporosity, as observed by transmission electron microscopy (TEM) with pore sizes of $5.6 \mathrm{~nm}$ and $S_{\mathrm{A}}=80 \mathrm{~m}^{2} \mathrm{~g}^{-1}$ in the case of 1-butyl-3-methylimidazolium dicyanamide. ${ }^{357}$ Yan et al. used melamine along with P123 for the synthesis of wormlike $\mathrm{CN}$ with a narrow pore size distribution; the utilization of Pluronic P123 also resulted in a certain carbon enrichment that shifted the absorbance edge up to $800 \mathrm{~nm}$. Consequently, the prepared material showed good photocatalytic HER activity with Pt as a cocatalyst and produced $148.2 \mu \mathrm{mol} \mathrm{h}^{-1} \mathrm{H}_{2}$ under visible light irradiation. Additionally, when the incident wavelength was longer than $700 \mathrm{~nm}$, the material still produced 3.6 $\mu \mathrm{mol} \mathrm{h}{ }^{-1} .^{358}$ A smaller templating agent (e.g., sucrose) was also used with melamine to produce mesoporous $\mathrm{CN}$. Upon thermal condensation, sucrose firstly melts and then decomposes, releasing carbon-based gases that induce porosity in the final CN. BET analysis confirmed the $S_{\mathrm{A}}$ enhancement up to $121 \mathrm{~m}^{2}$ $\mathrm{g}^{-1}$, which contributed to the enhancement of the photocatalytic HER activity. ${ }^{359}$ The utilization of biopolymers or biofibers as soft templating agents has been shown as another effective path to modify textural and photophysical properties. Zhang et al. used DCDA along with alginate or gelatin to modify the microstructure and electronic properties of $\mathrm{CN}$ and observed 5- and 6-fold $S_{\mathrm{A}}$ enhancement when using alginate and gelatin, respectively. An additional consequence of these conditions is the insertion of carbon atoms, which in turn results in unpaired electrons within the $\mathrm{CN}$ lattice, measurable using electron paramagnetic resonance (EPR). Furthermore, the prepared materials were tested as photocathodes in a photoelectrochemical cell, showing a 2.6-fold enhancement of the photocurrent which remained stable during repeated on/off cycles. ${ }^{360}$ Additionally, mesoporous tubular CN with carbon doping was obtained using kapok fiber as a templating agent along with urea. This novel approach modifies the band structure toward an enhanced optical absorption and fast charge carrier separation, resulting in enhanced HER photocatalytic performance. ${ }^{361}$ Recently, urea was also condensed with a melamine formaldehyde resin; the resulting $\mathrm{CN}$ shows a nanotube morphology with enhanced $S_{\mathrm{A}}$, hydrophilicity and light harvesting properties, which result in a remarkable HER performance, reaching a $19.2 \% \mathrm{AQY}^{362}$

\subsection{Ionothermal synthesis}

The crystallinity of $\mathrm{CN}$ materials is a key factor for an efficient charge transfer and for the separation of photoexcited states and therefore for the achievement of a high photocatalytic activity. Merschjann et al. and others have shown that the electronic transport in CNs occurs perpendicularly to the $2 \mathrm{D}$ sheets' plane, in contrast to the situation in graphene-related materials. $^{363,364}$ Therefore, achieving the closest possible, defect-free, highly ordered packing of the CN layers can substantially improve the electron and hole mobilities, one of the main bottlenecks of CN photocatalysts. Unlike the copolymerization of an organic monomer with a single alkaline salt, which typically results merely in a CN doped with the alkali metal or the anionic counterpart, ${ }^{365-370}$ using eutectic salt mixtures for the preparation of CNs induces both crystallinity and a higher degree of condensation in the resulting $\mathrm{CN}$ materials, and therefore has emerged as an effective approach to obtain high photocatalytic performance. ${ }^{371,372}$

A eutectic mixture is composed of two or more compounds that melt simultaneously at temperatures ranging from $100 \mathrm{up}$ to $1000{ }^{\circ} \mathrm{C}$, allowing a miscible multicomponent liquid phase for the synthesis of inorganic crystalline materials and carbonnitrogen based materials. ${ }^{373,374}$ Kuhn et al. described in 2008 the synthesis of triazine-based porous polymers in molten $\mathrm{ZnCl}_{2}$ that promoted the crystallinity of the framework. In this work the aromatic nitriles undergo dynamic trimerization within the ionic melt in a quartz ampule at $400{ }^{\circ} \mathrm{C}$ for $40 \mathrm{~h}$, reaching an almost complete conversion. Fourier-transform infrared (FTIR) spectroscopy and X-ray diffraction (XRD) confirmed the formation of a crystalline polymer with a hexagonal pore packing and a specific surface area and pore volume of $791 \mathrm{~m}^{2}$ $\mathrm{g}^{-1}$ and $0.4 \mathrm{~cm}^{3} \mathrm{~g}^{-1}$, respectively. ${ }^{375}$ The utilization of a salt-melt mixture for the synthesis of $\mathrm{CN}$ was shown in a report by Bojdys et al. in 2008, where the condensation of DCDA was carried out in a $\mathrm{LiCl} / \mathrm{KCl}$ mixture as the solvent. The $\mathrm{CN}$ obtained via this synthetic route shows an optimal degree of condensation, as proven by the absence of detectable amine groups in FTIR and corroborated by its high crystallinity reflected in the XRD 
patterns, where the (100) and (002) diffractions were observed at $12.1^{\circ}$ and $26.5^{\circ}$ respectively, corresponding to $d=7.3$ and $3.36 \AA$ and further supported by TEM. ${ }^{376}$ Later in 2011, Winhier et al. confirmed that the previously reported proposition of heptazine-based structures consists of a triazine-based framework, poly(triazine imide) (PTI). ${ }^{377} \mathrm{Li}^{+}$and $\mathrm{Cl}^{-}$intercalated PTI was prepared through the thermal treatment of DCDA in a eutectic mixture of $\mathrm{LiCl}$ and $\mathrm{KCl}$, and the resulting material consisted of 2D networks composed of planar layers based on imide-bridged triazine units with $\mathrm{Li}^{+}$and $\mathrm{Cl}^{-}$ions as confirmed by electron energy loss spectroscopy (EELS). ${ }^{15} \mathrm{~N}-\mathrm{NMR}$ confirmed the triazine-based structure and the absence of heptazine motifs. Poly(triazine imides) have been studied in depth ever since; in 2013, the use of $\mathrm{LiBr}$ and $\mathrm{KBr}$ for their synthesis expanded the $d$ spacing from 3.38 (observed in PTI prepared in $\mathrm{LiCl} / \mathrm{KCl}$ ) to $3.52 \AA$ and led to a theoretical band gap between 1.6 and $2.0 \mathrm{eV}^{378}$ Chong et al. have shown the replacement of $\mathrm{Br}^{-}$by $\mathrm{F}^{-}$ions by washing with concentrated ammonium fluoride, obtaining PTI with intercalated fluoride ions and a smaller interlayer distance of $3.32 \AA .^{379}$ The high photocatalytic performance of PTI materials was described later for both water half-splitting reactions with high $\mathrm{H}_{2}$ and $\mathrm{O}_{2}$ production rates. ${ }^{380-383}$ Further modification of the texture and electronic properties of the resulting material can be obtained by using organic monomers in order to enhance their optical absorption $^{384}$ and by combining metallic salts with different loadings ${ }^{385}$ (e.g., $\mathrm{NaCl}, \mathrm{CuCl}_{2}, \mathrm{ZnCl}_{2}$ and others). ${ }^{386-389}$ In 2018, Heymann $e t$ al. showed the electrochemical synthesis of PTI by coupling melamine radicals in a three-electrode setup; this novel approach allows the synthesis of PTI materials without intercalated ionic species. Additionally, despite the well-known high dispersibility of PTI, ${ }^{390}$ in this work, the high solubility of the obtained product allowed its analysis using liquid-state NMR. ${ }^{391}$

By selecting a novel monomer with a condensation temperature lower than that of the standard $\mathrm{C}-\mathrm{N}$ based reactants, Dontsova et al. prepared well-defined potassium poly(heptazine imides) (PHTIK). Here, substituted triazoles were used along with a $\mathrm{KCl} / \mathrm{LiCl}$ mixture. Given the temperature of the first reaction step $\left(300{ }^{\circ} \mathrm{C}\right)$ and the melting point of the eutectic mixture $\left(352^{\circ} \mathrm{C}\right)$, the structure of the final material was directed towards a crystalline, heptazine-based structure ${ }^{113}$ with the capability to reduce water and exchange ions within the solid state. ${ }^{392}$ Since this first report on the ionothermal synthesis of PHTIK from triazole derivatives, several reports have been published on the modification of the properties of the final CN material produced from different reactant molecules ${ }^{393-395}$ or metallic salts. ${ }^{396}$ Their remarkable performances in photooxidation processes and catalyzing organic syntheses have also been described. ${ }^{397-399}$ Besides using raw $\mathrm{C}-\mathrm{N}$ monomers with a condensation temperature lower than the melting point of the eutectic mixture, thermally treating common organic molecules to obtain melon units before the ionothermal synthetic step has emerged as an approach for the synthesis of highly photoactive materials. Lin et al. preheated melamine at $500^{\circ} \mathrm{C}$ to prepare tri$s$-triazine units (or $s$-heptazines ${ }^{400}$ ) and further heated the product in a $\mathrm{KCl} / \mathrm{LiCl}$ mixture in order to achieve highly
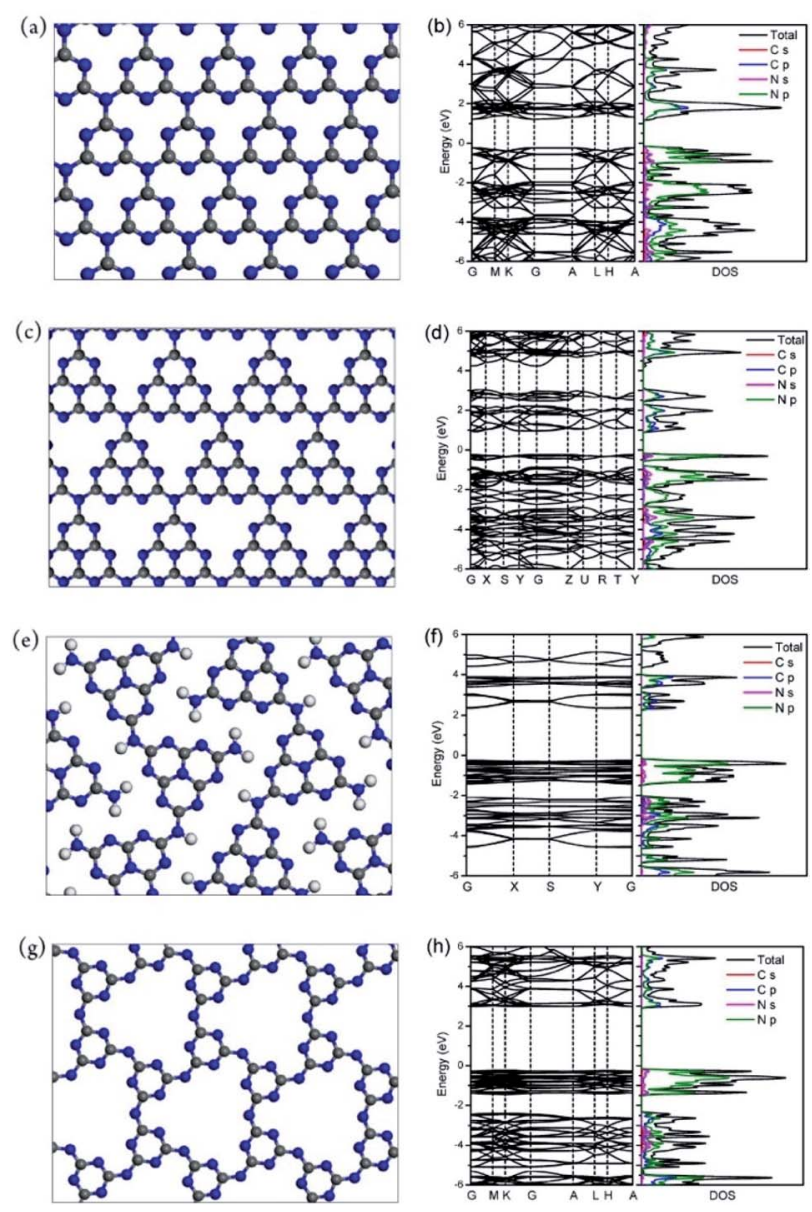

Fig. 10 Models and electronic structure of different $\mathrm{CN}$ structures. (a and b) Triazine-based, (c and d) tri-s-triazine-based, (e and f) melon and ( $g$ and $h$ ) PTI. Reproduced with permission from ref. 401, copyright (2016) American Chemical Society.

crystalline $\mathrm{CN}$. Theoretical and experimental results confirmed the improved photogenerated charge carrier mobility of the tri$s$-triazine-based CN vs. PTI, or melon type (Fig. 10), which resulted in a remarkable $50.7 \%$ AQY in the HER under $405 \mathrm{~nm}$ light illumination in a natural photosynthetic environment. ${ }^{401}$ Zhang et al. used a copolymerized CN material derived from urea and oxamide for the synthesis of a highly crystalline network based on heptazine units in a $\mathrm{LiCl} / \mathrm{KCl}$ mixture. The narrowed interlayer distance and enhanced light absorption result in significant hydrogen production with a $57 \%$ and $10 \%$ $\mathrm{AQY}$ at 420 and $525 \mathrm{~nm}$, respectively. ${ }^{402}$

The variety of $\mathrm{CN}$ structures that can be obtained via ionothermal synthesis and the optimization of synthetic parameters (e.g., monomer-to-salt ratio or condensation path) has allowed, amongst others, the formation of isotype triazineheptazine heterojunctions. They typically enhance the lifetime of photogenerated charge carriers thanks to the band alignment between different electronic structures, thereby promoting the photocatalytic activity; ${ }^{403,404}$ see also Section 5.4.

In 2017 , Jin et al. polymerized urea in various amounts of the eutectic mixture $\mathrm{LiCl} / \mathrm{KCl}$ at $450-550{ }^{\circ} \mathrm{C}$. Temperatures above 
$500{ }^{\circ} \mathrm{C}$ resulted in PTI structures given their high thermal stability compared to heptazine structures. Nevertheless, at a fixed synthesis temperature of $500{ }^{\circ} \mathrm{C}$, the salt-to-urea ratio determines the structural transformation from PTI into heptazines: a high molar ratio of molten salt to urea promotes the formation of a triazine-based structure, while a moderate ratio favors the heptazine form. The PTI/heptazine structure was confirmed by XRD, TEM and solid-state NMR and its photocatalytic performance (for the HER in this case) was substantially enhanced by the improved charge separation at the isotype heterojunction (Fig. 11). ${ }^{405}$ The presence of cations in the $\mathrm{CN}$ material resulting from an ionothermal synthetic procedure resulted in varying photocatalytic HER performance when salts were added to the reaction mixture. Li et al. polymerized melamine in the presence of a KCl-LiCl eutectic mixture and observed that the addition of certain cations, such as $\mathrm{Rb}^{+}$or $\mathrm{K}^{+}$, promotes the assembly of the CNK material into nanorods and furthermore boosts the photocatalytic activity due to a dielectric screening effect. ${ }^{406}$

\subsection{Supramolecular design}

The use of supramolecular structures as a tool for the rational design of CNs has attracted widespread attention during the last 7 years, due to the possibility of imprinting novel morphologies and electronic structures from the molecular level all the way to the final product after calcination. In this approach, C-N-based monomers interact by non-covalent interactions, forming versatile self-assembled supramolecular frameworks of different dimensionalities in a given solvent. Their features strongly depend on the intermolecular forces at play (hydrogen and halogen bonding, $\pi-\pi$ stacking, electrostatic interactions, etc.). ${ }^{407,408}$ The thermal condensation of the described supramolecular structures at high temperatures (300-650 ${ }^{\circ} \mathrm{C}$ ) can induce the morphology and the monomer sequence onto the final $\mathrm{CN}$ material, thereby allowing the rational design of properties for a given application while surpassing the drawbacks of solid-state reactions. ${ }^{409}$

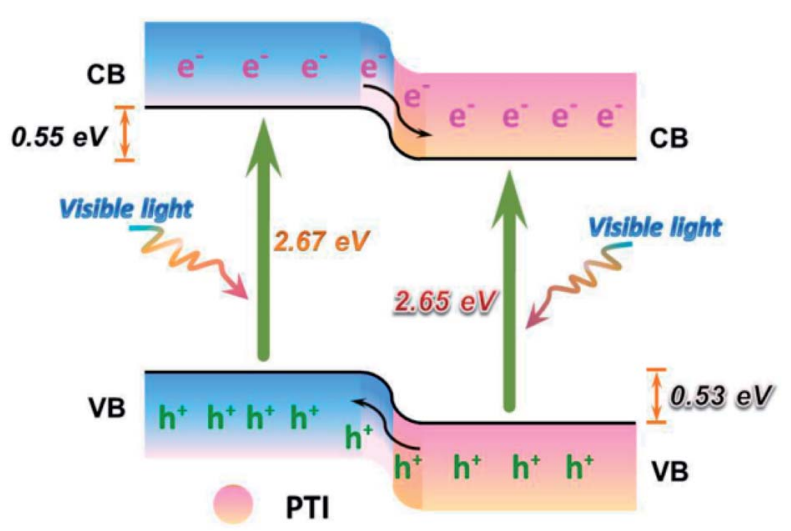

Heptazine based CN

Fig. 11 Charge separation at a PTI/heptazine $\mathrm{CN}$ heterojunction. Reproduced with permission from ref. 405, copyright (2017) American Chemical Society.
4.5.1. Acidic treatment of organic precursors. The protonation of organic monomers with halogen acids is a versatile tool for the generation of supramolecular assemblies of different dimensionalities. Melamine, for example, after protonation with a halogen acid, generates organic microsheets through counterion-bridged hydrogen bonds, electrostatic interactions and $\pi-\pi$ stacking. ${ }^{\mathbf{4 1 0 , 4 1 1}}$ Consequently, inducing a certain kind of dimensionality and electronic properties in a pristine supramolecular assembly can promote a fine control over the morphology and charge transport of the final CN material (after the thermal condensation at $300-650{ }^{\circ} \mathrm{C}$ ). The first insight into the effect of the treatment of organic precursors with strong acids and its utilization for the synthesis of $\mathrm{CN}$ materials was provided by Yan et al., who treated melamine with sulfuric acid before thermal condensation; they observed an enhanced thermal stability of the supramolecular arrangement. At high temperatures, sulfur was partially retained, which promoted charge separation upon illumination and HER photocatalytic activity. ${ }^{\mathbf{4 1 2}}$

The formation of a supramolecular assembly between sulfuric acid and melamine was further explored by others, confirming the quenching of sublimation during thermal treatment, the enhanced porosity, and the presence of trace amounts of sulfur, thereby modifying the chemical composition in the final bulk material, which promotes the photoactivity in various model reactions. ${ }^{\mathbf{4 1 3 , 4 1 4}}$ Hydrochloric acid is the most commonly used strong acid for the synthesis of $\mathrm{C}-\mathrm{N}$-based supramolecular assemblies that serve as reactants for a high temperature solid-state reaction, and a large amount of work has been carried out since the first reports, showing the possibility of tuning the morphology and photoactivity of the final $\mathrm{CN}$ by using melamine hydrochloride as the starting reactant. ${ }^{415-418}$

Our group and others studied the effect of chloride ions on the features of the initial supramolecular assembly with melamine. ${ }^{419}$ Upon protonation, melamine is arranged in a $3 \mathrm{D}$ fashion with a repeating unit in plane, ${ }^{\mathbf{4 2 0}}$ and this structure has also been observed in other supramolecular structures, where treatment with $\mathrm{HCl}$ induces directionality. ${ }^{\mathbf{4 2 1}}$ The morphology imprinted by the supramolecular assembly strongly alters the physical features of the $\mathrm{CN}$ material, promoting the formation of ultrathin nanosheets or enhanced porosity. ${ }^{\mathbf{4 2 2}}$ Furthermore, Zhang et al. confirmed the strong alteration of the band structure, which results in a positive shift of the $\mathrm{CB}$ and a negative shift of the $\mathrm{VB}^{423}$ Bromide can also direct supramolecular assemblies based on melamine; our group showed that the sequential treatment of melamine with $\mathrm{HCl}$ and $\mathrm{HBr}$ results in the selective coordination of each halogen acid at a different binding site, namely an amine group or pyridinic nitrogen. Additionally, molecular dynamics simulations showed a stronger interaction of both amine and pyridinic $\mathrm{N}$ with $\mathrm{Br}$ rather than with $\mathrm{Cl}$; therefore the modifications imprinted by $\mathrm{HBr}$ in the melamine- $\mathrm{HCl}$ supramolecular assembly strongly alter its chemical nature and dimensionality. The latter CN material showed a novel dot-like morphology with remarkable performance for the HER, reaching an AQY of 3.2\% and 2.1\% 
a)

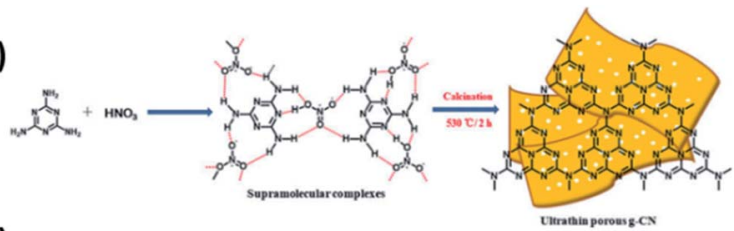

b)

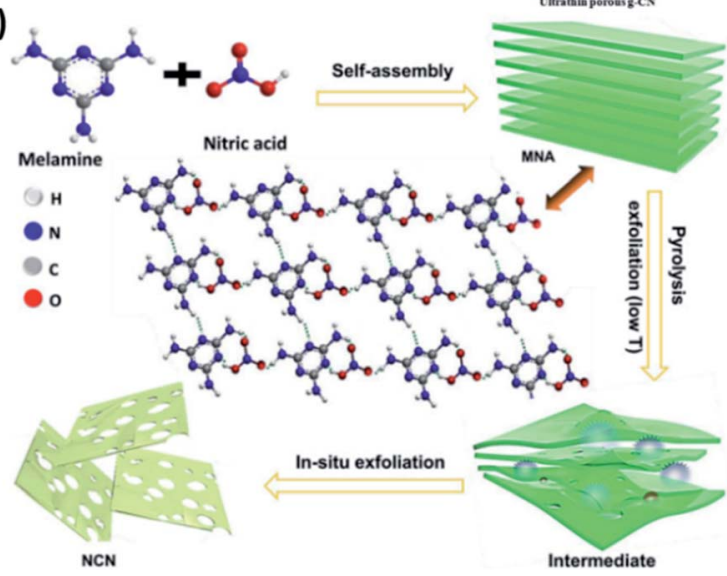

Fig. 12 Synthesis of a supramolecular complex based on melamine and nitric acid and its conversion into porous $\mathrm{CN}$ nanosheets. (a) Reproduced from ref. 425, copyright (2019) Materials Research Society. (b) Reproduced from ref. 434, copyright (2019) Elsevier publishing group.

upon illumination at 405 and $455 \mathrm{~nm}$, respectively. ${ }^{424}$ Treating melamine with nitric acid $\left(\mathrm{HNO}_{3}\right)$ is one of the most effective approaches to direct the growth of the prepared $\mathrm{CN}$ towards one direction. Melamine and nitric acid interact by hydrogen bonding, forming supramolecular complexes with a rod-like shape and different sizes depending on the acid concentration or reaction time (Fig. 12) ${ }^{425}$ The rod-like morphology of the supramolecular assembly (melamine- $\mathrm{HNO}_{3}$ ) can effectively imprint a 1D morphology on the final CN material after condensation, as shown for the first time by Tahir et al. in $2014 .{ }^{426}$ Other groups have shown the application of $1 \mathrm{D}$ carbon nitrides, prepared using this method, in different catalytic scenarios such as dye photodegradation, an electrocatalytic ORR or a photocatalytic HER. ${ }^{427-430}$ Furthermore, several groups have studied the self-assembly of melamine with different strong acids and their combinations; $\mathrm{CN}$ obtained from selfassembled melamine- $\mathrm{HNO}_{3}$ proved to have the highest photocatalytic performance. ${ }^{431,432}$ Additionally, the use of supramolecular assemblies comprising melamine and nitric acid has allowed the tailoring of the chemical composition and electronic structure by further inserting heteroatoms, ${ }^{433}$ promoting the exfoliation of $\mathrm{CN}$ into ultrathin films (Fig. 12b), ${ }^{434}$ and generating homojunctions that improve the charge separation efficiency. ${ }^{435}$ Furthermore, while the protonation of standard $\mathrm{C}-\mathrm{N}$ monomers before the pyrolysis does not typically modify the bulk chemical composition of the $\mathrm{CN}$ polymers, the use of acids containing heteroatoms can successfully alter it by inserting a given dopant element. Weng et al. and others described the synthesis of $\mathrm{BN}$ and $\mathrm{BCN}$ nanosheets from a hydrogen-bonded framework precursor containing melamine and boric acid after pyrolysis at $1050{ }^{\circ} \mathrm{C},{ }^{436,437}$ where hydrogen bonds are established between the hydroxyl groups of boric acid and both the amine and pyridinic nitrogens of melamine. ${ }^{438}$

4.5.2. Hydrogen bonding between $\mathbf{C}-\mathbf{N}$ monomers. Jun et al. reported the use of hydrogen-bonded frameworks based on the self-assembly of different $\mathrm{C}-\mathrm{N}$ building blocks for the synthesis of nanostructured CN. In their work, melamine was preorganized with cyanuric acid in dimethylsulfoxide (DMSO), resulting in flower-like supramolecular spheres, which after thermal treatment form hollow CN spheres with enhanced photocatalytic activity for RhB photodegradation and the HER. ${ }^{439,440}$ The cyanuric acid-melamine lattice (CM) contains up to 3 different type of hydrogen bonds and can adopt several forms depending on the solvent used for its preparation; this supramolecular assembly has been known for a long time and is one of the most utilized platforms for the synthesis of supramolecular structures. ${ }^{441-444}$ The versatility of the CM assembly and the possibility of modifying its nature by using different solvents or suitable $\mathrm{C}-\mathrm{N}$ monomers have allowed its exploitation as a common reactant for $\mathrm{CN}$ synthesis with tailored chemical composition, electronic properties, morphology and dimensionality. ${ }^{409}$

The possibility of fine-tuning the CM lattice by using different solvents was confirmed, and thus the porosity and electronic properties of the resulting CNs were optimized towards enhanced photocatalytic activity. Nevertheless, due to the chemical composition of melamine and cyanuric acid and the release of both amine groups and $\mathrm{O}$ atoms, the chemical composition of the final $\mathrm{CN}$ polymer remains unaltered. ${ }^{445}$ Since the first reports on the utilization of CM lattices for the preparation of nanostructured $\mathrm{CN}$, the modification of $\mathrm{CM}$ assemblies for the preparation of various $\mathrm{CN}$ materials with novel electronic structures and morphologies along with their versatile applications have been widely explored, which resulted in the accumulation of a vast body of knowledge. ${ }^{446-449}$

Inserting suitable $\mathrm{C}-\mathrm{N}$ monomers modifies the dimensionality of the CM supramolecular assembly and the electronic properties of the final $\mathrm{CN}$ material by increasing the C-to-N ratio, which effectively narrows the optical band gap; small organic molecules, such as barbituric acid, ${ }^{450-452}$ aromatic moieties, ${ }^{453-456}$ urea, ${ }^{457}$ caffeine, ${ }^{458}$ nucleobases ${ }^{459}$ and others, ${ }^{460-463}$ have been shown to undergo supramolecular assembly with melamine and cyanuric acid, thus enhancing the photocatalytic activity of the final $\mathrm{CN}$ material (Fig. 13a). Furthermore, modulating the CM assembly with heteroatomcontaining molecules allows the successful inclusion of the desired heteroatom within the framework of the final CN material, thereby modifying its bulk composition; boric acid or phosphoric acid can be integrated within the CM by hydrothermal treatment and preserve a certain amount of the doping agent, which promotes the charge transfer and separation of photoexcited states (Fig. 13b). ${ }^{464-466}$ Additionally, the utilization of sodium persulfate $\left(\mathrm{Na}_{2} \mathrm{~S}_{2} \mathrm{O}_{8}\right)$ along with the in situ generated $\mathrm{CM}$ lattice results in an enhanced porosity of the final CN, thanks to the release of sulfur-containing species, which improves the photo-electrochemical performance. ${ }^{467}$ 

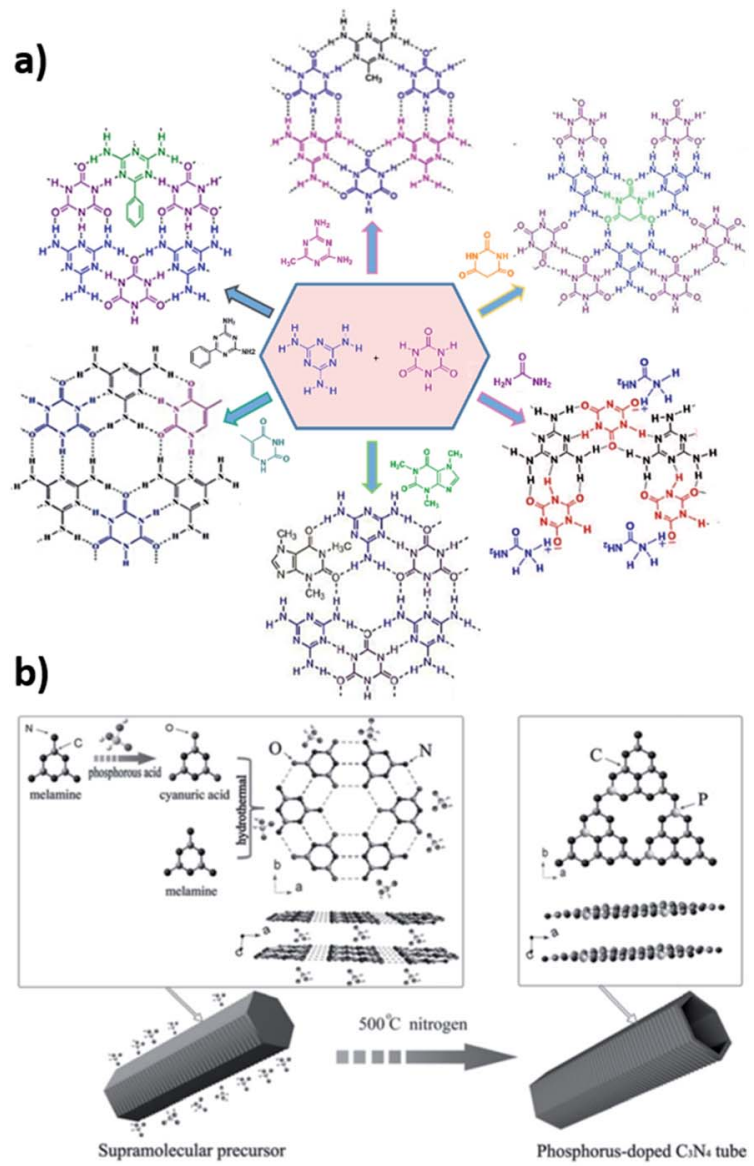

c)

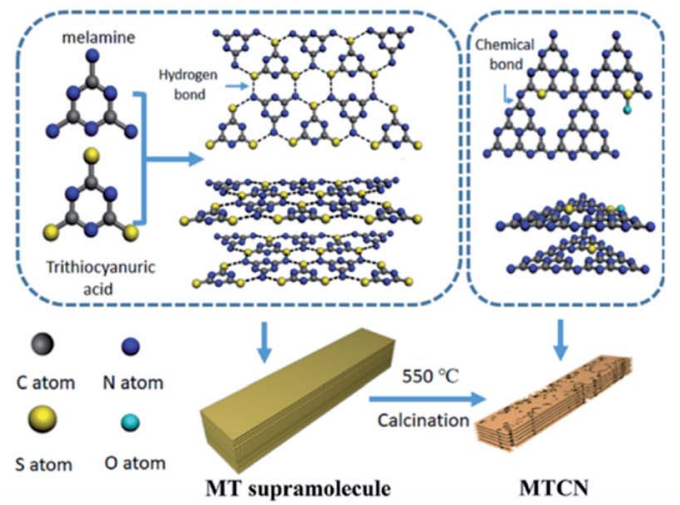

Fig. 13 (a) Supramolecular structures based on the cyanuric acidmelamine lattice. Reproduced from ref. 409, copyright (2018) Wiley$\mathrm{VCH}$. (b) Synthesis of phosphorus-doped CN nanotubes. Reproduced from ref. 464, copyright (2016) Wiley-VCH. (c) Synthesis of a supramolecular assembly between melamine and trithiocyanuric acid and its conversion to sulfur-doped CN. Reproduced from ref. 473, copyright (2018) Elsevier publishing group.

The replacement of melamine with a similar C-rich triazinelike molecule in the CM lattice modifies the chemical and electronic properties of $\mathrm{CN}$. Xu et al. showed that when substituting a phenyl-modified triazine-based molecule such as benzoguanamine for melamine, a molten state was induced during the thermal condensation, which allowed the formation of homogeneous films of C-rich $\mathrm{CN}$ polymers over conductive substrates, opening the door to its application in solar cells. ${ }^{468}$ Additionally, the resulting phenyl-modified CN material, upon exfoliation, yields CN QDs with a remarkable PL behavior. ${ }^{469}$ Other C-rich molecules such as triaminopyrimidine (very similar to melamine but with one carbon replacing a nitrogen in the triazine ring), when self-assembled with cyanuric acid (CA), lead to the formation of $\mathrm{C}_{3} \mathrm{~N}_{3} \mathrm{O}$ materials after thermal condensation at low temperatures, with photocatalytic activity for dye degradation, the HER and $\mathrm{CO}_{2}$ reduction. ${ }^{84}$

In addition to the widespread utilization of the $\mathrm{CM}$ lattice as a versatile platform for the design of $\mathrm{C}-\mathrm{N}$ based supramolecular structures, melamine and other $\mathrm{C}-\mathrm{N}$ monomers can also selfassemble with a wide variety of suitable monomers; this results in a diversity of supramolecular structures that modify the final $\mathrm{CN}$ properties towards an enhanced photoactivity. Trithiocyanuric acid (TTC), for example, has been widely used along with melamine for the synthesis of sulfur-doped $\mathrm{CN}$, as shown by Feng et al. ${ }^{470}$ After this report, several groups have reported the formation of a M-TTC supramolecular assembly and its conversion into S-doped CN, with sulfur atoms typically replacing carbon in the $\mathrm{CN}$ framework; they used the latter for different photocatalytic scenarios including dye degradation, ${ }^{471}$ reduction of $\mathrm{Cr}(\mathrm{vI}),{ }^{472}$ the $\mathrm{HER}^{473}$ and formation of liquid crystals (Fig. 13c). ${ }^{474}$ Additionally, a large number of small organic molecules have been utilized and self-assembled with melamine; urea, ${ }^{475}$ glucose, ${ }^{476}$ oxalic acid, ${ }^{477}$ chitosan $^{478}$ and others $^{479-481}$ can interact by hydrogen bonding with melamine and form supramolecular aggregates, which after thermal treatment preserve certain C-doping. The clever selection of the building blocks taking part in the supramolecular self-assembly permits the obtention of semiconducting domains with a nonnegligible photoactivity at low temperatures $\left(250^{\circ} \mathrm{C}\right)$; benzoquinone derivatives or chloranilic acid are some of the species that, after assembly with melamine and condensation at mild temperatures, have resulted in semiconducting polymers with photocatalytic activity in dye degradation and water oxidation..$^{482,483}$

Additionally, several reports have performed the selfassembly of melamine with inorganic salts and explored the features of the final $\mathrm{CN}$. Chen et al. preorganized melamine with $\left[\mathrm{Al}\left(\mathrm{NO}_{3}\right)_{3} \cdot 9 \mathrm{H}_{2} \mathrm{O}\right]$, which interacts via hydrogen bonding between the amine groups of melamine and the water molecules of the hydrated complex. The thermal condensation of the supramolecular arrangement results in aluminum oxide species that can be removed using an acidic wash, resulting in enhanced $S_{\mathrm{A}}$ and photocatalytic activity. ${ }^{484}$ Following this report, ionic complexes such as ammonium oxalate or hydroxylammonium chloride have been proven to form a supramolecular precursor with melamine by hydrogen bonding between amino groups and carbonyl or hydroxyl groups. The thermal condensation of these supramolecular precursors directs the morphology of the final $\mathrm{CN}$ towards porous nanotubes and enhanced photocatalytic activity in the HER or $\mathrm{CO}_{2}$ reduction. ${ }^{485,486}$

4.5.3. Solvent-directed supramolecular assemblies. Solvents play a decisive role in the aggregation shape and size of supramolecular assemblies. In the case of a hydrogen-bonded 
framework, a polar solvent can disrupt the molecular forces and interact with the monomeric units, thus inducing a complete rearrangement. Meanwhile, a non-polar solvent, which cannot participate directly in hydrogen bonding, may just imprint surface modifications on the aggregate. ${ }^{\mathbf{4 8 7 - 4 9 0}}$ Solvents can also serve as building blocks in the formation of supramolecular assemblies due to their hydrogen bonding sites; for example, water, hydrogen peroxide and polyethylene glycol have all been shown to self-assemble with melamine and different supramolecular frameworks, resulting in the modification of the chemical composition, morphology and photophysical properties of the final $\mathrm{CN}$ materials. This approach has led to oxygen doping, the formation of homogeneous $\mathrm{CN}$ films and an overall enhancement in photocatalytic activity. ${ }^{491-494}$ As another example, the treatment of DCDA with $N, N$-dimethylformamide (DMF) before the thermal condensation results in the generation of $\mathrm{N}$ vacancies that promote the separation of photoexcited charges and enhance the photocatalytic activity. ${ }^{495}$

In the case of $\mathrm{CM}$ lattices, the solvent determines the aggregation mode of the supramolecular assembly and therefore the properties of the CN. In 2013, Shalom et al. prepared a CM lattice in different solvents, namely water, chloroform and ethanol. They observed that given the different surface energies of the hydrogen-bonded structures formed the aggregate displayed different morphologies, namely rods, needles and pancake-like structures, which introduced different structural motifs and photophysical properties into the final CN. ${ }^{\mathbf{4 5} 5}$ Later this phenomenon was further studied in CM-barbituric acid (CMB) networks. A supramolecular CMB assembly in water yields a rod-like morphology, while in ethanol, spheres were formed. Upon thermal condensation, macroscopic (up to 4$5 \mathrm{~cm}$ long) $\mathrm{CN}$ wires grow spontaneously from the supramolecular spheres, whose properties strongly differ from the substrate, resulting in two distinct $\mathrm{CN}$ populations that can be used for water purification or $\mathrm{H}_{2}$ production through water splitting. ${ }^{496}$ Furthermore, recently Wang et al. studied the variation of the hydrogen-bond lengths in a CM framework and its effect on the resulting $\mathrm{CN}$ material. The self-assembled precursors were freeze-dried in order to decrease the length and increase the strength of the hydrogen bonds. ${ }^{497,498}$ Upon calcination, the distorted supramolecular structure results in a nanocage-like $\mathrm{CN}$ with enhanced $S_{\mathrm{A}}$, which can photocatalyze $\mathrm{CO}_{2}$ reduction. ${ }^{499}$ For heteroatom-containing supramolecular assemblies, our group has shown that the solubility of the initial monomers in a given solvent can dramatically alter their inclusion within the hydrogen-bonded framework. We have shown that preorganizing melamine with 1,3,4-thiadiazole-2,5dithiol in DMSO results in a higher integration of the $\mathrm{S}$ containing moiety in the starting assembly, which implies a more defined structural rearrangement compared to the assemblies prepared in water or common organic solvents. The unique chemical composition and structure achieved when using DMSO as the solvent are induced in the final CN material, resulting in a trace amount of sulfur doping, a beneficial electronic structure for photocatalysis and an enhanced $S_{\mathrm{A}} \cdot{ }^{\mathbf{5 0 0}}$

The manipulation of $\mathrm{CM}$ lattices by sequential solvent treatment also allows the formation of thermodynamically driven gradients of energy levels; Sun et al. prepared CM supramolecular assemblies in various solvents and confirmed the stronger aggregation of CM lattices in acetone and ethanol. Additionally, the prepared assemblies were subsequently immersed in a second solvent and this solvent's capability to disrupt the hydrogen bonding between the monomers was explored. As expected, polar solvents could penetrate within the structure, causing a complete rearrangement, while non-polar solvents modify only the aggregate's surface, which in turn leads to the formation of two interconnected CNs with an energy band gradient after thermal treatment, enhancing the lifetime of the photogenerated electron-hole pairs and the overall photocatalytic activity. ${ }^{501}$ Nevertheless, the introduction of a second solvent for the synthesis of supramolecular structures that serve as $\mathrm{CN}$ reactants is still in its infancy, and nowadays, most assemblies are still prepared in only one solvent. This fact limits the library of available monomers to those dispersible in a common solvent. However, in polymer chemistry and other fields, the advantages of using two nonmiscible solvents have been demonstrated for the interfacial polymerization of supramolecular structures, allowing the interaction of several different units dispersed in non-miscible solvents (Fig. 14a). ${ }^{502}$ This concept was recently utilized in our group for the synthesis of CM frameworks. Specifically, we have shown the formation of CM frameworks in water-chloroform

a)

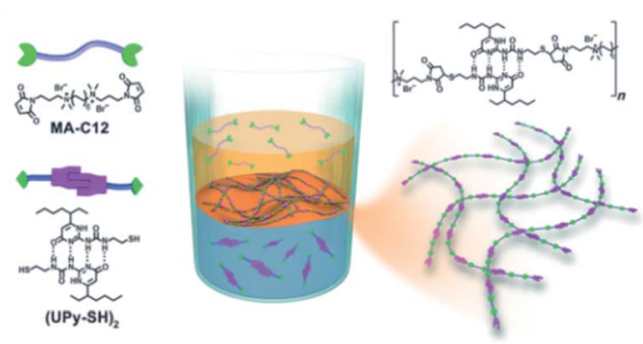

b)

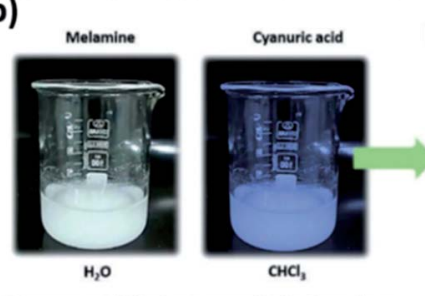

Monomers diffusion towards the interface
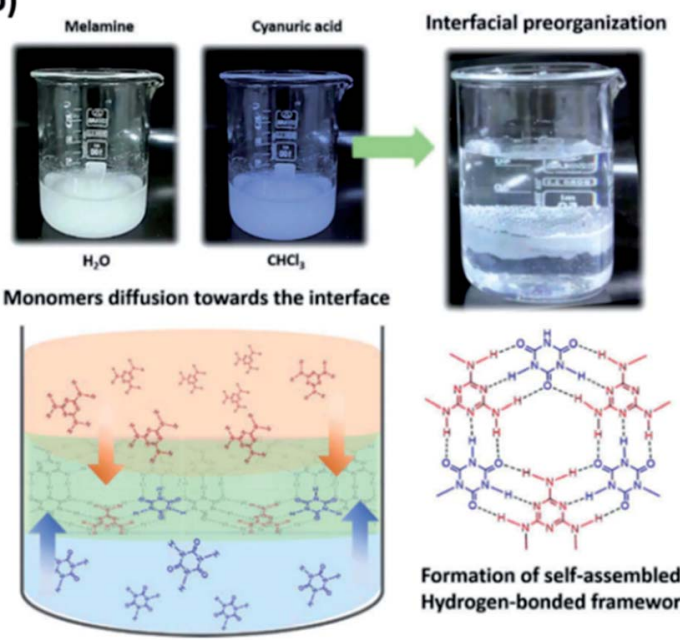

Formation of self-assembled Hydrogen-bonded frameworks

Fig. 14 (a) Schematic representation of the interfacial supramolecular polymerization. Reproduced from ref. 502, copyright (2017) Wiley$\mathrm{VCH}$. (b) Synthesis of cyanuric acid-melamine (CM) frameworks in a water-chloroform interfacial (immiscible) system. Reproduced from ref. 503, copyright (2019) Royal Society of Chemistry. 
interfacial systems. Molecular dynamics simulations showed that both monomers tend to interact at the interface rather than in the bulk of either solvent. This thermodynamic tendency induces a complete rearrangement of the morphology. The thermal condensation of this system results in hollow CN tubes with altered electronic properties. ${ }^{503}$ Additionally, this approach allows a straightforward modification of CM units, either in situ by drop-casting a dopant monomer (such as picric acid from the aqueous phase, which inserts $\mathrm{C}-\mathrm{C}$ bonds within the final $\mathrm{CN}$ polymer) or by preorganizing melamine before the mixing of the immiscible phases (water and chloroform, Fig. 14b). Particularly, Dolai et al. used a monomer with both hydrogen-bond donor and acceptor sites (i.e., the amino acid creatine) to selectively bind melamine and CA at the interface, thereby directing the modified CM units towards one direction. The latter $\mathrm{CN}$ material shows an altered morphology and electronic properties, which promote its photocatalytic activity towards several model reactions. ${ }^{504}$

4.5.4. Supramolecular single crystals. The use of supramolecular single crystals has recently been proven to be a very accurate method to tailor the morphology and chemical composition of the prepared $\mathrm{C}-\mathrm{N}$ based materials. A single crystal is a solid with a defined repetitive chemical composition and a long-range atomic order and does not contain grain boundaries. The growth of molecular single crystals can be carried out in several manners, and the solvent most often plays a crucial role: slow solvent evaporation, liquid diffusion, gas diffusion and others. ${ }^{505,506}$ The synthesis of supramolecular single crystals based on $\mathrm{C}-\mathrm{N}$ monomers as building units followed by their thermal condensation at elevated temperatures has been recently exploited as a very reliable approach to precisely control the properties of the final $\mathrm{C}-\mathrm{N}$ based materials. ${ }^{507}$ The first reports describing the synthesis of a melaminebased ordered supramolecular framework and its utilization as a reactant for a high temperature solid-state reaction included the formation of a metal-organic network between melamine and $\mathrm{Cu}(\mathrm{II})$ species in different solvents; it yielded threedimensional porous structures with hexagonal pores linked by $\mathrm{N}-\mathrm{H} \cdots \mathrm{N}$ hydrogen bonds. The thermal condensation of such structures resulted in nanosheets and nanorods with residual $\mathrm{Cu}$ doping and decoration with $\mathrm{CuO} .^{\mathbf{5 0 8 , 5 0 9}}$

In 2018 this concept was established as a tool for the rational design of $\mathrm{CN}$ properties; our group showed the synthesis of melaminium chloride hemihydrate single crystals, where protonated melaminium residues are connected by chloride ions and water molecules. The crystals exhibited a 1D needle shaped morphology, which after thermal treatment was induced in the final $\mathrm{CN}$ material, resulting in macroscopic centimeter-long $\mathrm{CN}$ needles suitable for optoelectronic applications. Additionally, the chemical and photophysical properties of the final material could be fine-tuned by the reaction temperature (up to $650{ }^{\circ} \mathrm{C}$ ) as well as by the chemical composition of the starting crystal through the insertion of a C-rich monomer such as benzoguanamine. ${ }^{\mathbf{5 1 0}}$ Several groups have exploited novel single-crystal structures based on triazine molecules for the synthesis of modified CN materials. Zhu et al. used crystalline copper chloride templated by protonated melamine with the form $\left[\mathrm{H}_{2} \text { mela }\right]_{2}\left[\mathrm{CuCl}_{5}\right] \mathrm{Cl}$, consisting of
$\left[\mathrm{CuCl}_{5}\right]_{n}{ }^{3 n-}$ chains with $\mathrm{Cl}^{-}$anions and layers of doubly protonated melaminium cations $\left(\left[\mathrm{H}_{2}\right.\right.$ mela $\left.]\right)$, bridged by $\mathrm{N}-\mathrm{H} \cdots \mathrm{Cl}$ hydrogen bonds. ${ }^{511}$ The pyrolysis of this precursor allowed the release of $\mathrm{HCl}(\mathrm{g})$, which favors the formation of $\mathrm{Cu}-\mathrm{N}$ bonds and results in the successful inclusion of up to $25.9 \mathrm{wt} \%$ of $\mathrm{Cu}$ within the final CN material. Furthermore, they studied the crystal preorganization effect by comparing the resulting material to the one obtained by the simple copolymerization of melamine with a metallic salt, concluding that the formation of highly ordered crystals promotes the inclusion of metallic $\mathrm{Cu}^{2+}$, whereas in other scenarios CuO NPs were formed on the CN surface. The photocatalytic activity was substantially enhanced as a result of the capability of $\mathrm{Cu}-\mathrm{N}$ bonds to produce hydroxyl radicals which efficiently degrade standard organic pollutants such as RhB, MB or MO. ${ }^{512}$ Jiang et al. further used a wide variety of metal chlorides $\left(\mathrm{Fe}^{3+}, \mathrm{Ni}^{2+}, \mathrm{Co}^{2+}\right.$ and $\left.\mathrm{Mn}^{2+}\right)$ for the synthesis of metal-organic crystal precursors; the latter were obtained by slowly cooling a saturated solution containing melamine and the metal chloride. The use of the highly crystalline precursors for the synthesis of CN resulted in the formation of nanotubes with enhanced photocatalytic activity for RhB degradation and the HER. ${ }^{513}$ Besides metal-organic crystals, using co-crystals formed by non-covalent interactions between different organic monomers can lead to a novel layered morphology, as well as a certain degree of carbon doping in the CN. Fang et al. showed the formation of co-crystals comprising terephthalic acid (TPA) and melamine, which interact by hydrogen bonding between the carboxylic groups of TPA and the amines of melamine, and their utilization as precursors for high temperature reactions. The hydrogen-bonded framework is organized as 2D molecular sheets. Additionally, the aromatic character of TPA allows the preparation of carbon-rich frameworks with a long range order, thereby promoting the charge separation of photogenerated charge carriers in the final $\mathrm{CN}$ material. The resulting C-rich material showed simultaneous $\mathrm{H}_{2}$ and $\mathrm{O}_{2}$ production, with an AQY of $5.3 \%$ for the HER. ${ }^{514}$ Additionally, Zhang et al. utilized a 3D hydrogen bonded crystal obtained by the recrystallization of urea from DMF. Upon thermal condensation, the DMF within the crystal structure is released promoting the formation of nanosheets with an enhanced surface area and photocatalytic performance..$^{515}$

\section{Post-synthetic modifications}

Despite the relatively easy manipulation of CN parameters from the synthetic point of view, where electronic properties, texture and surface chemistry can be tailored, the post-synthetic modification of these materials is more complicated given the stability and low dispersibility of $\mathrm{CN}$ polymers in most solvents. The polarity of the $-\mathrm{C}=\mathrm{N}$ - bond within the lattice, or the remaining $-\mathrm{NH}_{2}$ groups, a result of non-ideal condensation, permits the tailoring of the electronic properties and structure in a post-synthetic manner. Since covalent transformations within the $\mathrm{CN}$ structure are complex, several means have emerged in order to induce doping or defects, which improve the charge transfer and the photocatalytic performance, including consecutive thermal treatments, ${ }^{516-521}$ hydrothermal and solvothermal means, ${ }^{522-529}$ protonation $^{530-532}$ and others. ${ }^{533}$ 
In this section, the focus is on the recent developments in postsynthetic modifications of $\mathrm{CN}$, as well as the formation of hybrid composites with other layered materials, NPs, molecular complexes and so forth.

\subsection{Covalent functionalization}

Covalent chemistry offers a wide range of possible organic motifs to include within $\mathrm{CN}$ frameworks; the electronic properties and other parameters, such as colloidal stability or surface chemistry, are strongly affected by the attached functional group. Furthermore, this approach allows the formation of nanostructures and features, which is not possible to achieve by other conventional means. ${ }^{534}$ The first studies involving covalent functionalization of $\mathrm{CN}$ frameworks were performed by taking advantage of the electron lone pairs of the $-\mathrm{NH}_{2}$ terminal groups; in 2010, Guo et al. described the amidation of 1,2,4,5benzene tetracarboxylic dianhydride (PMDA) and the CN amine groups for the extension of the polymer conjugation towards improved visible-light harvesting properties. First principles calculations confirmed that the PMDA moiety is perpendicular to the $\mathrm{CN}$ plane, promoting the repulsion between $2 \mathrm{D}$ layers, and ${ }^{13} \mathrm{C}-\mathrm{NMR}$ proved the presence of carbonyl carbons in the imide group. The improved conjugation leads to extended light absorption and promotes photocatalytic activity as demonstrated for MO photodegradation. ${ }^{535}$ In a similar manner, the conjugation was further extended by the introduction of perylene imidies into the $\mathrm{CN}$ framework, which promoted the photocatalytic removal of nitric oxide. ${ }^{536,537}$

Lotsch and co-workers screened a wide variety of heptazine units functionalized in the 2, 5 and 8 positions with different moieties (e.g., alkyl chains, $p$-tolyl, $p$-benzoic acid, melonates and triphtalimide). The melonate form, obtained by salt-melt synthesis, improves the coordination to a platinum co-catalyst by cyanamide defects and therefore enhances the chargetransfer efficiency, resulting in a HER with 9.3\% AQY ${ }^{538}$ The introduction of cyano terminal groups through different synthetic pathways was also investigated by several groups showing a general enhancement of the photocatalytic activity thanks to the modification of the electronic structure. In these studies, the materials were prepared by mild treatment with a strong reducing agent such as $\mathrm{NaBH}_{4}$ or photoinduced in the presence of thiocyanates. ${ }^{539,540}$ Further treatment of the melonates with $\mathrm{HCl}$ yields urea-modified CNs through the hydrolyzation of the cyanamide defect group. This library of materials was shown to produce hydrogen through the water splitting reaction even in the absence of a Pt co-catalyst. When Pt was loaded, an AQY of $18 \%$ was obtained with methanol as a sacrificial electron donor, one of the highest photocatalytic activities for polymeric photocatalysts. ${ }^{\mathbf{5 4 1}}$

Schiff-base chemistry involving terminal $-\mathrm{NH}_{2}$ groups has been widely used to prepare CNs with extended conjugation and enhanced photocatalytic performances. ${ }^{542}$ The thermal treatment at mild temperatures of $\mathrm{CN}$ with various aromatic carboxylic acids has been demonstrated to successfully graft the organic moiety to the solid polymer by Tian et al., who synthesized aromatic heterocycle-grafted CNs and observed that the electronic properties and photocatalytic performance strongly depended on the structure of the formed copolymer. ${ }^{543}$ The conjugation of $\mathrm{CN}$ frameworks through terminal amino groups can also modify their semiconductor behavior. Vidyasagar et al. successfully grafted $\mathrm{CN}$ with 2,5-thiophenedicarboxylic acid. The introduction of the organic moiety, proven by XPS and solid-state NMR, strongly modifies the band structure of $\mathrm{CN}$, yielding both $\mathrm{n}$ - and $\mathrm{p}$-type semiconducting domains, probed by Mott-Schottky analyses. The minimization of $\mathrm{e}^{-}-\mathrm{h}^{+}$recombination enhanced both photocatalytic activity and chargecapacitance behavior. ${ }^{544}$ Besides small conjugated molecules or functional groups, the $-\mathrm{NH}_{2}$ groups in the edge planes of $\mathrm{CN}$ also allow covalent bonding to larger molecular materials or graphitic nanostructures; Pan et al. covalently embedded heptazine units within a covalent-organic framework (COF) by reacting them with 1,3,5-triformyl phloroglucinol and 2,4,6-tris (4-aminophenyl)-1,3,5-triazine in DMSO (Fig. 15a). ${ }^{545} \mathrm{Lu}$ et al. immobilized Zn-phthalocyanine on CN through amide bonding in order to improve the light absorption and overall photocatalytic performance in organic dye degradation. ${ }^{546}$ The same effect has been observed with covalently bonded porphirins to $\mathrm{CN}$, which promote light harvesting and lead to a remarkable HER and $\mathrm{CO}_{2}$ reduction performance. ${ }^{547,548}$ The amidation reaction has also been utilized for the covalent bonding of $\mathrm{CN}$ to carbon nanotubes (CNTs), which promoted the electron transfer in the ORR and the photocatalytic activity for $\mathrm{H}_{2} \mathrm{O}_{2}$ production (Fig. 15b). ${ }^{549}$

Besides the covalent functionalizations taking advantage of the lone pair of terminal amine groups, cycloadditions, alkylations and other reactions have been carried out on $\mathrm{CN}$ by making use of the polar $-\mathrm{C}=\mathrm{N}$ - bond within the framework. In 2014, Zhang et al. carried out the 1,3-dipolar cycloaddition reaction of azomethine ylides, known as Prato's reaction, for

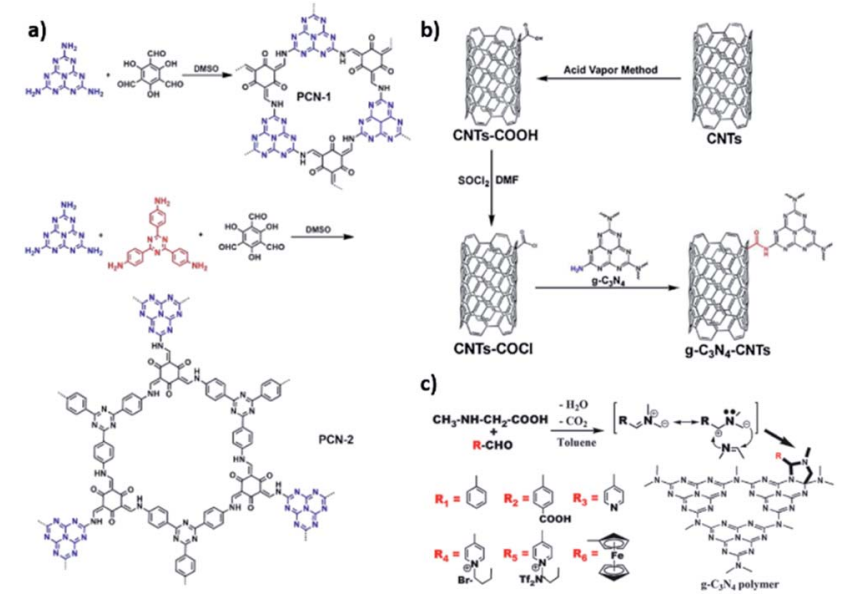

Fig. 15 Covalent functionalization schemes. (a) Synthesis of heptazine-functionalized covalent organic frameworks (COFs). Reproduced from ref. 545, copyright (2018) Wiley-VCH. (b) Synthesis of covalently bonded CN to a carbon nanotube. Reproduced from ref. 549, copyright (2018) Elsevier publishing group. (c) 1,3-Dipolar cycloaddition reactions for the covalent functionalization of $\mathrm{CN}$. Reproduced from ref. 550, copyright (2014) Royal Society of Chemistry. 
the functionalization of $\mathrm{CN}$ frameworks with a wide library of organic groups, including aryls, ionic liquids, ferrocene and more (Fig. 15c). In this work, the reaction takes place in toluene at $120{ }^{\circ} \mathrm{C}$ for $21 \mathrm{~h}$, where azomethine ylide is first generated, in situ, by condensation of an $\alpha$-amino acid and an aldehyde bearing the functional group to be inserted. The electronic structure of functionalized $\mathrm{CN}$ was tailored according to the functional group and a general light-absorption enhancement was observed. The photocatalytic activity was tested for the $\mathrm{C}-\mathrm{H}$ oxidation reaction of $\beta$-isophorone to ketoisophorone, where the $\mathrm{CN}$ functionalized with ferrocene achieved a 96\% conversion with $82 \%$ selectivity. ${ }^{550}$ Graphitic nanostructures like fullerenes can also be covalently bonded to $\mathrm{CN}$ by a solid-state mechanochemical route; Chen et al. utilized ball-milling to functionalize $\mathrm{CN}$ with a $\mathrm{C}_{60}$ fullerene in the presence of lithium hydroxide ( $\mathrm{LiOH})$ through the fourmembered ring of azetidine.

The $\mathrm{CN} / \mathrm{C}_{60}$ hybrid was used as a photocatalyst for the hydrogen evolution reaction under visible light with eosin $\mathrm{Y}$ as a sensitizer, without a noble-metal cocatalyst, showing a 4-fold increase in activity over pristine $\mathrm{CN}$ due to electron transfer from the sensitizer to the hybrid system and an improved charge separation efficiency. ${ }^{551}$ The $\mathrm{C}=\mathrm{N}$ bond reactivity within $\mathrm{CN}$ also allows the covalent bonding of alkyl chains, forming a new $\mathrm{C}-\mathrm{C}$ bond by using alkyl halides (the $\mathrm{C}=\mathrm{N}$ bond serves as an excellent radical acceptor in this alkyl halide radical mechanism). The inclusion of such chains promotes the exfoliation of $\mathrm{CN}$ layers, by improving their solubility in common solvents such as tetrahydrofuran (THF). ${ }^{52}$ Related mechanisms can use dihalides and allow further functionalization with bio-compatible molecules as quinoxalines for organocatalysis. ${ }^{553}$

The chemical oxidation of $\mathrm{CN}$ nanosheets through acidic hydrothermal treatment has also been studied as a feasible way to improve the solubility of the material and its reactivity toward further functionalization. ${ }^{554}$ Along these lines, Teng et al. synthetized edge-functionalized CN via Hummers' method. ${ }^{555}$ Mulliken charge distribution confirmed that the surface acid groups improve the charge separation efficiency, thereby facilitating the formation of hydrogen peroxide and displaying a remarkable performance in the photocatalytic disinfection of water. A more complex covalent functionalization has been carried out utilizing halogenated organic monomers for the synthesis of $\mathrm{CN}$ frameworks modified with functional groups. Our group demonstrated that the calcination at $350{ }^{\circ} \mathrm{C}$ of a triazine-based molecule modified with halogenated aromatics, prepared by a reaction between DCDA and halogenated benzonitriles, yields CN frameworks with photophysical properties strongly dependent on the functional group. ${ }^{173}$ The added halogenated group serves as a highly active chemically reactive center, which allows its substitution by phenyl and tert-butyl propionate groups via Suzuki and reductive-Heck cross-coupling reactions, respectively. Other complex modifications of $\mathrm{CN}$ nanosheets promote their exfoliation and dispersibility in common solvents and furthermore enhance their photocatalytic activity towards the oxidation of benzyl alcohol as a model reaction. ${ }^{556}$

The capability of the $\mathrm{C}=\mathrm{N}$ bond in the heterocyclic ring to act as a radical acceptor in intermolecular alkyl radical additions allows the formation of $\mathrm{CN}$-based hydrogels $(\mathrm{CN}-\mathrm{H})$ through the photoinduced generation of radicals and introduction of cross-linkers, functional groups, etc. The photoinduced grafting of molecules over $\mathrm{CN}$ polymers has been proven to permit the introduction of moieties such as fluoride or sulfonic groups as well as polymeric chains that modify the electronic structure, texture and colloidal properties of $\mathrm{CN}$, toward its application in multiple fields. ${ }^{557-562}$ Furthermore, the formation of $\mathrm{CN}$-based hydrogels with a defined 3D shape which maintain the semiconducting features of the matrix while surpassing its intrinsic drawbacks (grain boundaries or fast recombination) is an approach that has lately gathered attention. The tunability of the morphology, mechanical strength and photophysical properties of CN-based hydrogels through the selection of functional groups and cross-linkers may permit their application on an industrial scale in a wide range of fields. ${ }^{563-565}$

Thus far, the reports on $\mathrm{CN}-\mathrm{H}$ were mostly based on noncovalent interactions with external agents, such as peptides, ${ }^{\mathbf{5 6 6}}$ ionic liquids, ${ }^{567}$ graphitic materials ${ }^{568-571}$ and others. ${ }^{572-574}$ Typically, they involve the self-assembly of CN nanosheets by $\pi-$ $\pi$ or hydrogen-bonding interactions to form the desired $3 \mathrm{D}$ networks. Photopolymerization in the presence of cross-linkers has been used to covalently functionalize the surface of $\mathrm{CN}$
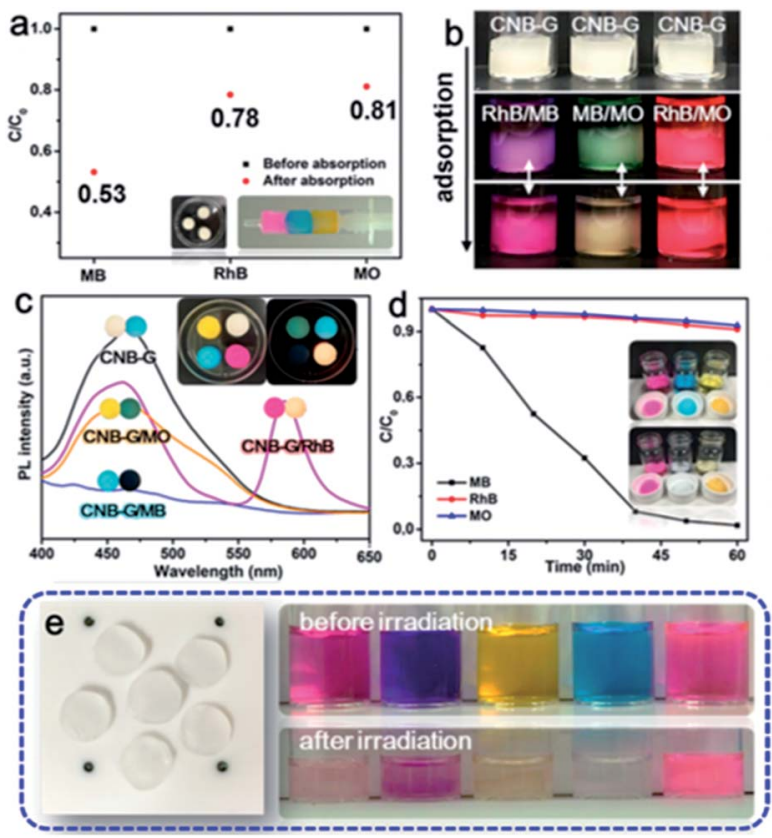

Fig. 16 Application of $\mathrm{CN}$-based hydrogels $(\mathrm{CN}-\mathrm{H})$ for dye adsorption and degradation. (a) Adsorption capacity with different organic dyes, (b) digital images of the $\mathrm{CN}-\mathrm{H}$ adsorption capacity with mixed dyes, (c) photoluminescence spectra before and after dye adsorption, (d) photodegradation of $\mathrm{CN}-\mathrm{H}$ of $\mathrm{MB}, \mathrm{RhB}$ and $\mathrm{MO}$, and (e) digital images of the photodegradation of various dyes with $\mathrm{CN}-\mathrm{H}$. Reproduced from ref. 575, copyright (2017) American Chemical Society. 
networks and form 3D structures; it yields robust $\mathrm{CN}-\mathrm{H}$ with remarkable mechanical and photocatalytic properties. In 2017, Sun et al. have shown that $\mathrm{CN}$ generates surface radicals that induce in situ polymerization of acrylamide derivatives that yield photoactive $\mathrm{CN}-\mathrm{H}$. The resulting materials show strong mechanical strength and deformation restorability; these hydrogels were applied for the adsorption and degradation of organic dyes and the photocatalytic HER (Fig. 16). ${ }^{575}$ Liu et al. used poly( $N$-isopropylacrylamide) (NIPA) and $\mathrm{CN}$ for the formation of $\mathrm{CN}-\mathrm{H}$; the gelation occurred by photocatalytic generation of hydroxyl radicals, which initiate the polymerization. Furthermore, the temperature-responsive character of NIPA allowed the formation of smart coatings with tailorable turbidity by temperature control. ${ }^{576}$ The colloidal properties of the $\mathrm{CN}$ used for the formation of $\mathrm{CN}-\mathrm{H}$ has been proven to be crucial for the modulation of the final mechanical properties. For example, Kumru et al. showed that $\mathrm{CN}-\mathrm{H}$ derived from urea$\mathrm{CN}$ with a zeta potential of $-40.9 \mathrm{mV}$ displays a storage modulus at $0.1 \%$ strain of $8320 \mathrm{~Pa} .{ }^{577}$ Sulfonic acid was also grafted on $\mathrm{CN}$ and served both as the reinforcer and the initiator, resulting in $\mathrm{CN}-\mathrm{H}$ with enhanced compressibility, tissue adhesive properties and moderate flexibility. ${ }^{578}$ The same authors also reported the effect of the amount of $\mathrm{CN}$ used in the preparation of $\mathrm{CN}-\mathrm{H}$ in an ethylene glycol (EG) dispersant. Thus, they could achieve dispersions with up to $4 \mathrm{wt} \%$ of $\mathrm{CN}$, yielding hydrogels with a remarkably high storage modulus of $720 \mathrm{kPa} .{ }^{579}$ Ye et al. showed a wide range of novel applications for $\mathrm{CN}-\mathrm{H}$. Specifically, they used $\mathrm{CN}-\mathrm{H}$ obtained through photopolymerization of $\mathrm{CN}$ with acrylamide. The prepared hydrogels showed good mechanical properties as well as self-healing capabilities, and their performance as a UV irradiation protective film was tested for the first time and showed 95\% filtered UV radiation $(315-400 \mathrm{~nm}) .{ }^{580}$

\subsection{Exfoliation}

The exfoliation of layered materials is an established approach for the obtention of isolated flakes or 2D nanosheets. The exfoliation of a bulk layered material into thin sheets increases the number of active sites and therefore its reactivity. Furthermore, the choice of the exfoliation technique, namely mechanical, thermal or chemical (in a liquid phase), defines the yield and the morphology of the nanosheets, thus determining their performance in a given application. ${ }^{581}$ In particular, liquidphase exfoliation has been one of the most widespread techniques due to the possibility of weakening the van der Waals (vdW) interactions between the $2 \mathrm{D}$ sheets in layered materials through the insertion of surfactants and ions, and the possibility of modulating the exfoliation degree by controlling parameters such as shear force, temperature or ultrasonic frequency. ${ }^{582}$

Various 2D layered materials (graphene $,{ }^{583} \mathrm{BN},{ }^{584}$ transition metal dichalcogenides, ${ }^{585}$ and others ${ }^{586-588}$ ) have been successfully exfoliated using such techniques.

The exfoliation of $\mathrm{CN}$ is of great interest given the potential synthetic prospects and applications of nanosheet-based devices. CN layers interact by weak vdW forces, which can be easily disturbed by mechanical means. $\mathrm{CN}$ in the form of nanosheets is typically endowed with a significantly higher $S_{\mathrm{A}}$ and more abundant surface active sites, a larger $E_{\mathrm{g}}$ thanks to quantum confinement effects, and improved electron transport along the in-plane direction, which allows the enhancement of intrinsic properties, such as photoresponse. ${ }^{\mathbf{5 8 9}, 590}$

Zhang et al. showed the exfoliation of $\mathrm{CN}$ in solvents with various polarities and observed that the relatively higher polarity of water benefited the exfoliation; ultrathin sheets were obtained at a concentration of $0.15 \mathrm{mg} \mathrm{mL}^{-1}$ and with a zeta potential of $-30.3 \mathrm{mV}$. Furthermore, the $\mathrm{CN}$ layers in solution had diameters of 70-160 $\mathrm{nm}$ and a thickness of $2.5 \mathrm{~nm}$. This suspension remained stable for months even at $\mathrm{pH}$ values ranging from 3 to 11 , with the electronic structure strongly altered compared to the bulk counterpart. DFT calculations confirmed that single-layered $\mathrm{CN}$ has an increased density of states at the CB edge compared to the bulk-an indication of a higher charge-carrier concentration-which supports the measurement of an increased photoresponse. ${ }^{591}$ Yang et al. further studied the liquid-phase exfoliation of $\mathrm{CN}$ in solvents (including 2-propanol (IPA), NMP, water, ethanol and acetone) and the influence of sonication time, as well as solvent-material interactions. They found that NMP and IPA were the most appropriate solvents, given their surface energies $\left(40 \mathrm{~mJ} \mathrm{~cm}{ }^{-2}\right.$ vs. $70 \mathrm{~mJ} \mathrm{~cm} \mathrm{~cm}^{-2}$ for vdW-bonded surfaces). After $10 \mathrm{~h}$ of sonication, the $\mathrm{CN}$ dispersion consisted of $2 \mathrm{~nm}$-thick nanosheets that remained stable up to 4 months. The XRD pattern of the thin-layered material slightly differs from that of the bulk counterpart; the relative intensity of the (002) diffraction peak, corresponding to the interplanar stacking, significantly decreases. Additionally, the $\mathrm{CN}$ nanosheets showed a band gap of $2.65 \mathrm{eV}$ and a $3.75 \% \mathrm{AQY}$ in the HER owing to the high $S_{\mathrm{A}}$ and the resulting larger number of exposed catalytic sites. ${ }^{592}$

Since the pioneering reports of liquid-phase exfoliation of $\mathrm{CN}$, different groups have established relationships between the utilization of different solvents, including alcohols, ${ }^{593}$ sulfonic group-containing solvents, ${ }^{594,595}$ hydrogen peroxide, ${ }^{596}$ and others ${ }^{597,598}$ with the nanoscale features of the prepared sheets. ${ }^{599}$ The polymerization degree of the $\mathrm{CN}$ used in the exfoliation strongly determines the photophysical behavior of the prepared nanosheets, as shown by Zhou et al., who developed electrochemiluminescent sensors for the detection of multiple metal ions based on $\mathrm{CN}$ nanosheets with different condensation degrees that display different responses to each metal ion. ${ }^{600}$ The thermal exfoliation of $\mathrm{CN}$ can also yield $2 \mathrm{~nm}$ thick nanosheets with $S_{\mathrm{A}} \sim 300 \mathrm{~m}^{2} \mathrm{~g}^{-1}$. This procedure can be carried out by the thermal condensation of pre-formed $\mathrm{CN}$ at high temperatures and in air, which typically results in the oxidation of exposed nitrogen atoms. ${ }^{\mathbf{6 0 1 , 6 0 2}} \mathrm{Niu}$ et al. confirmed that the electronic properties of such nanosheets are also strongly altered as shown by a $0.2 \mathrm{eV}$ increase of $E_{\mathrm{g}}(2.97 v s$. 2.77 ), which correlated with a blue shift of the fluorescence emission maximum of $20 \mathrm{~nm}$. These quantum confinementderived effects are also reflected in the good electron conductivity along the in-plane direction of the nanosheet compared to its bulk counterpart and the enhanced photocatalytic activity for the generation of hydroxyl radicals. ${ }^{603}$ Additionally, Xia et al. 
established that the atmosphere used during the thermal exfoliation can induce chemical modifications in the nanosheets, for example, amine functionalization by thermal exfoliation of bulk $\mathrm{CN}$ under a continuous $\mathrm{NH}_{3}$ flow. ${ }^{604}$ In order to reduce the thickness of the discussed 2 nm-thick $\mathrm{CN}$ nanosheets and obtain single atomic monolayers, a chemical exfoliation of bulk $\mathrm{CN}$ can be performed, as reported in 2013 by $\mathrm{Xu}$ et al.; bulk CN was stirred in the presence of $\mathrm{H}_{2} \mathrm{SO}_{4}(98 \mathrm{wt} \%$ ) for $8 \mathrm{~h}$, followed by a liquid-phase exfoliation in water and reflux in methanol at $65{ }^{\circ} \mathrm{C}$ for $6 \mathrm{~h}$. The intercalation of $\mathrm{H}_{2} \mathrm{SO}_{4}$ within the $\mathrm{CN}$ layers resulted in a shift of the (002) diffraction peak down to $25.0^{\circ}$ indicating the expansion of the inter layer distance to $0.356 \mathrm{~nm}$; the latter exfoliation yielded $0.4 \mathrm{~nm}$-thick layers with tens of nanometers lateral size. The modification of the dimensionality strongly affected the optical properties, as the $E_{\mathrm{g}}$ increases from 2.64 to $2.92 \mathrm{eV}$ given the decrease in conjugation and the quantum confinement effect (also confirmed by a fluorescence emission peak blue shift from 464 in the bulk to 438 $\mathrm{nm})$. The modification of the nanosheet morphology and electronic properties resulted in an enhanced photocatalytic HER and photodegradation of phenol. ${ }^{605}$

The utilization of strong acids has been further studied by several groups, which reported the chemical cleavage of the $\mathrm{CN}$ framework, thus enhancing its solubility, which even allowed recording liquid-state NMR spectra. ${ }^{\mathbf{6 0 6}, 607}$ The exfoliation of
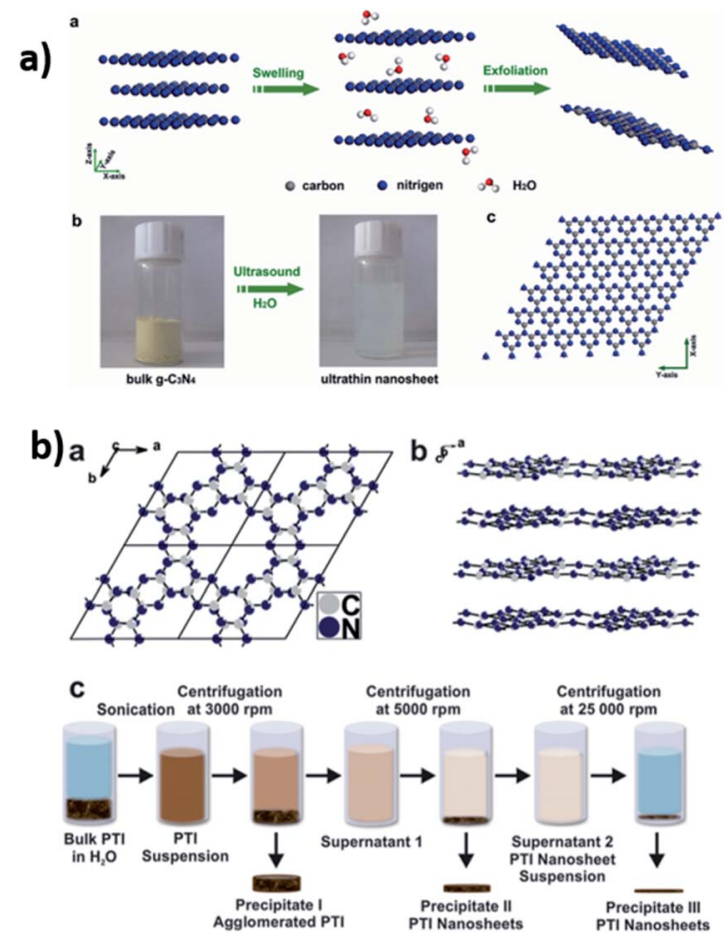

Fig. 17 (a) Scheme of the synthesis of dispersed $\mathrm{CN}$ nanosheets by liquid-phase exfoliation (a swelling step, followed by exfoliation using ultrasonication as an external mechanical force). Reproduced from ref. 591, copyright (2012) American Chemical Society. (b) Scheme of the preparation of ultrathin PTI materials by sequential exfoliation. Reproduced from ref. 613, copyright (2014) American Chemical Society. layered materials by mechanical means, e.g., scotch tape, which has been widely utilized, ${ }^{608-611}$ was also applied for CN. For example, Bojdys et al. showed the mechanical and chemical exfoliation of poly(triazine imide) with intercalated potassium metal that resulted in thin layers, scrolls and bundles with macroscopic lateral dimensions. Furthermore, the reported liquid-phase exfoliation resulted in atomically thin PTI sheets with $E_{\mathrm{g}}=2.6 \mathrm{eV} .^{612}$ The liquid-phase exfoliation of crystalline PTI materials was later studied by Schwinghammer et al., who showed that 1-2 nm-thick nanosheets of $\mathrm{PTI} / \mathrm{Li}^{+} \mathrm{Cl}^{-}$could be obtained by simple sequential ultrasonication in water (Fig. 17). The well-dispersed PTI nanosheets remained stable in both acidic and alkaline environments and showed improved photocatalytic HER performance. ${ }^{613}$ Further work on the exfoliation of PTI materials resulted in the complete dissolution of $\mathrm{PTI} \cdot \mathrm{Li}^{+} \mathrm{Br}^{-}$in the presence of polar aprotic organic solvents such as NMP, DMF or DMSO, with mass concentrations ca. 0.8 $\pm 0.05 \mathrm{mg} \mathrm{mL}^{-1}$. The spontaneous dissolution occurred as a result of a free energy gain upon solvent coordination, where the energies of the interlayer interactions remain similar to that of the solvent-nanosheet interface. ${ }^{\mathbf{6 1 4}}$

Utilizing intercalating agents to promote the separation of $\mathrm{CN}$ layers is a very effective approach to enhancing the yield of the nanosheets prepared via liquid-phase exfoliation. Xu et al. carried out the liquid-phase exfoliation of $\mathrm{CN}$ in the presence of ammonium chloride $\left(\mathrm{NH}_{4} \mathrm{Cl}\right)$; the thermal treatment of $\mathrm{CN}$ / $\mathrm{NH}_{4} \mathrm{Cl}$ resulted in the decomposition of ammonium ions, which distorted the vdW interactions between layers, resulting in 2-3 $\mathrm{nm}$ sheets with enhanced $S_{\mathrm{A}}$ and performance in the photoelectrochemical detection of copper(II) ions. ${ }^{615}$ Melamine has been utilized as an intercalating agent as well; Ma et al. treated $\mathrm{CN}$ with DMF and subsequently added melamine to achieve an optimal enlargement of the interlayer distance of $\mathrm{CN}$. The resulting material possessed an enhanced $S_{\mathrm{A}}(116.76$ $\mathrm{m}^{2} \mathrm{~g}^{-1}$ ), a larger bandgap (by $0.13 \mathrm{eV}$ ) and higher electron-hole mobility, which promoted the photocatalytic activity in a visible light-driven HER. ${ }^{616} \mathrm{Ji}$ et al. made the most of a planar conjugated molecule, such as 1-pyrene-butyrate, to exfoliate and modify the CN's surface via $\pi-\pi$ interactions. The latter was anchored to a DNA probe and used as an electrochemiluminescent DNA biosensor. ${ }^{617}$ Reversible chemical exfoliation was achieved by the intercalation of alkaline metal hydroxides and the re-assembly of CN layers. Li et al. utilized $\mathrm{OH}-$ modified $\mathrm{CN}^{618}$ for the in situ generation of water molecules upon acidic treatment, which promoted the exfoliation into thin nanosheets of 1-2 $\mathrm{nm}$ thickness and a yield of $48 \%$ at $\mathrm{pH}=$ 1.3. They were able to re-assemble the thin layers using a dilute alkali treatment; they exhibited a superior photocatalytic performance for IPA degradation and the HER thanks to their improved visible light absorption and hydrophilicity induced by the hydroxyl groups at the surface. ${ }^{619}$

\subsection{Hybrid composites based on $\mathrm{CN}$}

A hybrid composite (HC) combines the functionalities of its individual constituent domains. In our context, the novel properties result from the coupling between $\mathrm{CN}$ and the other 
domain(s). A metal domain may exhibit plasmonic effects and catalytic activities, while a semiconductor absorbs light, produces charge carriers and, depending on the band alignment at the SC-SC interface, can facilitate charge separation (in photovoltaics and photocatalysis) or recombination (in enhanced photoluminescence or light-emitting diodes). Such interfaces can exist on the macroscale, e.g., in CN films deposited on highly doped metal oxides or in the case of $\mathrm{CN}$ films deposited on various substrates with nanostructured features (vertically aligned $\mathrm{ZnO}$ nanorods, ${ }^{620}$ for example), or on the nanoscale as a post-synthetic modification of $\mathrm{CN}$, as discussed in this subsection (for example we refer the reader to a recent review from Liao et al., which summarizes many HCs used for the HER). ${ }^{146}$

5.3.1. Electronic coupling rationale in $\mathrm{CN}$-based hybrid composites. Fig. 18 presents the relevant possible electronic alignments (in the dark) of $\mathrm{CN}$ with another SC or metal. For the common application that is photocatalysis, a longer exciton lifetime increases the probability of the photoexcited charges to react with a given molecule; forming a heterojunction with suitable materials of different dimensionalities and band alignments has been shown to successfully quench the radiative recombination of electron-hole $\left(\mathrm{e}^{-}-\mathrm{h}^{+}\right)$pairs and therefore increase the yield in photocatalytic scenarios. ${ }^{621}$

In type-I heterojunctions, the $\mathrm{CB}$ and $\mathrm{VB}$ of one SC engulf the bands of the other (Fig. 18a, 'straddling gap'). Though this band alignment favors radiative recombination in the narrow- $E_{\mathrm{g}} \mathrm{SC}$, redox reactions can still take place, e.g., CN/antimonene, ${ }^{622} \mathrm{CN} /$ $\mathrm{ZnV}_{2} \mathrm{O}_{5}$ (ref. 623) and $\mathrm{CN} / \mathrm{ZnIn}_{2} \mathrm{~S}_{4} \cdot{ }^{624}$

In type-II heterojunctions, $\mathrm{e}^{-}$and $\mathrm{h}^{+}$spatial separation is favored across the formed interface, which is effective in
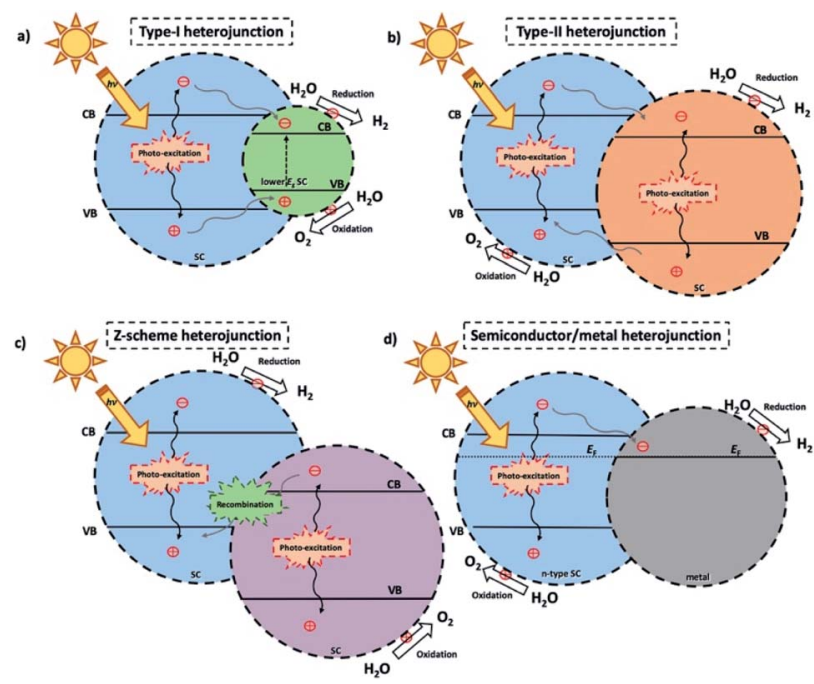

Fig. 18 Scheme of different heterojunctions based on a semiconductor heterointerface with another semiconductor: (a) Type-l, (b) Type-II, and (c) Z-scheme. (d) n-type SC interfaced with a metal ( $E_{\mathrm{F}}$ is the Fermi level), serving as the $\mathrm{e}^{-}$sink. In all cases, $C N$ serves as one SC (blue) and upon photoexcitation the electrons from its VB and holes from its $C B$ may migrate according to the band alignment. On the surface, electrons may perform a reduction reaction while holes perform an oxidation one. enhancing the lifetime of photogenerated charges (see photocatalytic examples in a recent review by Shen and co-workers). ${ }^{625}$ As $\mathrm{CN}$ is an n-type SC, the construction of a heterojunction with a p-type SC results in a built-in internal electric field, which helps drive charge separation, ${ }^{626}$ as was demonstrated using $\mathrm{BiOCl} / \mathrm{CN}^{627}$ and $\mathrm{Cu}_{2} \mathrm{O} / \mathrm{CN}^{628}$

Despite their efficiency in promoting charge separation, after the migration across the interface, the redox potential difference for a reaction at the surface is weakened in a type-II alignment (Fig. 18b, CB of the orange particle and VB of the blue SC). In order to maintain optimal reduction and oxidation potentials, while at the same time achieving the spatial charge separation of the photogenerated excitons, a Z-scheme charge transfer pathway between two semiconductors has emerged as an efficient strategy. ${ }^{629}$ In this case (Fig. 18c), ${ }^{630}$ photoexcited electrons from the 'purple' CB recombine with photogenerated holes of the 'blue' SC. In this manner, the remaining, spatially separated charges display optimal reduction and oxidation potentials as shown for various systems of $\mathrm{CN}$ with metal oxides, ${ }^{631-633}$ sulfides, ${ }^{634}$ etc. of different morphologies, where a continuous interface is present. The described Z-scheme can also be supplemented by a third material at the interface, an electron mediator such as metal NPs or reduced graphene oxide (rGO) ${ }^{630,635}$ An interesting combination of materials was achieved by Li and coworkers, who formed a Z-scheme catalyst where $\mathrm{CN}$ is the light absorber, meso-tetra(4-carboxyphenyl) porphyrin (TCPP) both acts as an antenna and provides excited electrons to perform the HER, and the third component of this hybrid is a $\mathrm{Cu} \mathrm{NP}$, which acts as the mediator. ${ }^{636}$ In this work, the intimate connection between $\mathrm{CN}$ and porphyrin, which is usually very weak owing to $\pi-\pi$ stacking and $\mathrm{H}$ bonding, was more robust thanks to the $\mathrm{Cu}$ linker.

Besides SC/SC junctions, interfacing CN with metal NPs, also known as Mott-Schottky junctions, has a catalytic effect in part due to a charge transfer between the SC and the metal. The metal NP, in turn, acts as a reactive binding site for a reduction or oxidation process. We recommend a thorough review by $\mathrm{Li}$ and Antonietti on this subject, where they discuss the different possible N-doped carbon/metal and CN/metal Mott-Schottky heterojunctions. ${ }^{637}$ Since $\mathrm{CN}$ is n-type, we limit our discussion to CN-to-metal electron transfer, which serves as an electron sink (Fig. 18d). This is relevant also for Z-scheme interfaces, where a Pt NP, for example, can catalyze the HER process using electrons from a $\mathrm{CN}$, thus enhancing the interfacial recombination at the direct Z-scheme interface $\left(2 \mathrm{D} \mathrm{CN}\right.$ sheets $/ 3 \mathrm{D} \mathrm{TiO}{ }_{2}$ microflowers), leaving behind holes in $\mathrm{TiO}_{2}$ that can oxidize water and/or hole scavengers as suggested by Wang et al. ${ }^{638}$

5.3.2. Interfacing $\mathbf{C N}$ with nanostructures. Metal-free carbon/carbon nitride junctions have attracted much attention due to the remarkable physicochemical properties of carbon allotropes such as high conductivity, significant $S_{\mathrm{A}}$, chemical stability and low price. ${ }^{639}$ Numerous composites of a conductive porous carbon matrix and $\mathrm{CN}$ have been reported. For example, a 3D carbon was prepared by chemical vapor deposition, followed by impregnation with urea and thermal 
polymerization, allowing the porous carbon to act as a template. ${ }^{\mathbf{6 4 0}}$

Graphene, carbon nanotubes or fullerenes can act as electron acceptors, promoting charge separation upon photoexcitation; they also successfully transfer the excited electrons to the reactant molecules. An intimate contact between the two counterparts can result in specific chemical bonds, such as covalent $\mathrm{C}-\mathrm{O}-\mathrm{C}$ moieties in the case of reduced graphene oxide (rGO) and $\mathrm{CN}$, which results in a narrowing of the band gap and an improvement in light-harvesting properties. ${ }^{641}$ Simple ultrasonication in toluene of $\mathrm{CN}$ powder and $\mathrm{C}_{60}$ results in a $\mathrm{CN} /$ $\mathrm{C}_{60} \mathrm{HC}$ tested for photocatalytic oxidation of (aromatic) sulfides to sulfoxides. ${ }^{\mathbf{6 4 2}}$

Other composites, which incorporate $\mathrm{CN}$, are constantly explored including different metal-free non-carbon-based 2D materials such as phosphorene ${ }^{\mathbf{6 4 3}}$ and antimonene, ${ }^{\mathbf{6 2 2}}$ due to their strong vdW interactions. ${ }^{621}$ Many other hybrids between $\mathrm{CN}$ and quasi-2D and other functional materials such as MOFs are reported; see for example, a review by Wang et al. ${ }^{\mathbf{6 4 4}}$

Metal-containing compounds can exhibit semiconductor or catalytic properties (or both). Here, we briefly survey some SC/ SC heterojunctions, while the catalysts are in the next subsection. The use of metal oxide materials is driven by their generally superior stability. Some recent examples include: the hydrothermal decoration of $\mathrm{CN}$ on rutile and anatase $\mathrm{TiO}_{2}$ nanotubes by Kumar et al.; ${ }^{645} \mathrm{CN}-\mathrm{WO}_{3}$ heterojunctions; ${ }^{635} \mathrm{Jiang}$ and co-workers' $\mathrm{CN}$ with a nanotube morphology decorated with $\mathrm{CoO}_{x}$ NPs $\left(\mathrm{CoO}\right.$ and $\left.\mathrm{Co}_{3} \mathrm{O}_{4}\right)$ for the HER, in which case they confirmed, using Kelvin-probe spectroscopy, that smaller $\mathrm{CoO}$ NPs (ca. $8 \mathrm{~nm}$ ) form a type-II heterojunction, while larger $\mathrm{Co}_{3} \mathrm{O}_{4}$ NPs $(\sim 10-50 \mathrm{~nm})$ form a type-I heterojunction with $\mathrm{CN} ;{ }^{\mathbf{6 4 6}}$ a bimetallic oxide $\left(\mathrm{NiCo}_{2} \mathrm{O}_{4}\right)$ on exfoliated $\mathrm{CN}$ sheets with enhanced HER performance owing to a formed junction between the p-type oxide and n-type $\mathrm{CN}$ showing superior performance relative to $\mathrm{NiO} / \mathrm{CN}$ and $\mathrm{Co}_{3} \mathrm{O}_{4} / \mathrm{CN}^{647} 2 \mathrm{D} / 2 \mathrm{D} \mathrm{CN} /$ $\mathrm{Co}_{3} \mathrm{O}_{4}$ or $\mathrm{CN} / \mathrm{V}_{2} \mathrm{O}_{5}$ for the HER; ${ }^{631}$ and $2 \mathrm{D} / 2 \mathrm{D}$ InSe/CN for the OER. ${ }^{648}$

Type II interfaces based on $\mathrm{CN}$ and metal chalcogenides are also common. A recent example of a complex morphology was demonstrated in the form of an octahedral CdS/CN hybrid for the HER and oxidation of toluene and other aromatic alkanes. In this case, first, a hydrothermal synthesis exploiting a coordination polymer with $\mathrm{Cd}-\mathrm{S}$ bonds was used as the precursor for the formation of $\mu \mathrm{m}$-sized porous CdS octahedra, which was then modified with melamine in an alkaline hydrothermal environment, finally forming a porous core-shell CdS structure with an external octahedral porous CdS shell/CN HC. ${ }^{649}$

5.3.3. Catalysts and co-catalysts: single atoms, molecular complexes, and metallic and other related NPs. As with other SC-based photocatalytic systems, a thermodynamic tendency towards charge separation is not sufficient. Since a significant kinetic barrier exists for most catalytic reactions occurring on a catalyst's surface (OER, various dissociation reactions, etc.), the SC (and SC/SC HCs) needs another catalyst or co-catalyst. ${ }^{162}$ Inspired by homogeneous organo-catalysis, various metalcontaining complexes have been coupled to SCs. Examples inspired by nature include hydrogenase enzymes, ${ }^{650,651}$ and horseradish peroxidase (HRP), which has an iron center that switches between Fe(III) and Fe(II) during a catalytic cycle was coupled to $\mathrm{CN}$ to enable the degradation of various phenolbased pollutants, doing so by increasing the concentration of ${ }^{\circ} \mathrm{OH},{ }^{\circ} \mathrm{O}_{2}{ }^{-}$and $\mathrm{H}_{2} \mathrm{O}_{2} \cdot{ }^{652,653}$ Specifically, a photoexcited $\mathrm{e}^{-}$from the $\mathrm{CN}$ can produce ${ }^{\circ} \mathrm{O}_{2}{ }^{-}$from dissolved oxygen, which reacts with $2 \mathrm{H}^{+}$(aq.) and dissolved oxygen to produce $\mathrm{H}_{2} \mathrm{O}_{2}$ (via 1 and 2-electron transfer processes, respectively), while $\mathrm{a} \mathrm{h}^{+}$can oxidize the pollutant phenol. The iron in HRP is reduced upon accepting an $\mathrm{e}^{-}$and regenerates to $\mathrm{Fe}(\mathrm{III})$ via a reaction with $\mathrm{H}_{2} \mathrm{O}_{2}$ with the release of ${ }^{\circ} \mathrm{OH}$ and $\mathrm{OH}^{-}$(HRP-Fe(II) $+\mathrm{H}_{2} \mathrm{O}_{2} \rightarrow$ $\left.\mathrm{HRP}-\mathrm{Fe}(\mathrm{III})+{ }^{\circ} \mathrm{OH}+\mathrm{OH}^{-}\right){ }^{652}$

Molecular complexes can be combined with $\mathrm{CN}$ in the liquid phase; for example, the boron-containing dye 4,4-difluoro-8phenyl-4-bora-3a,4adiaza-s-indacene (BODIPY) effectively extracts holes from $\mathrm{CN}$ in an OER scenario. ${ }^{531}$ Metal-containing catalysts such as $\mathrm{Ni}$ (II) bis(diphosphine) can act as $\mathrm{H}_{2}$-evolution catalysts in a photocatalytic system, where they are coupled to a CN (specifically, with a cyanamide surface functionalization), which performs selective oxidation of benzylic alcohols to aldehydes in water. ${ }^{654} \mathrm{CO}_{2}$ reduction and the HER using molecular catalysts such as $\mathrm{Co}(\mathrm{bpy})_{3}{ }^{2+}$ connected to $\mathrm{CN}$ using a molecular bridge were also reported. ${ }^{655}$

The extreme case of minimization would be the use of single atoms on the CN's surface. ${ }^{656-660}$ In this architecture, the $\mathrm{CN}$ acts as the support for the metal. It is extremely hard to prove the dispersion of single atoms and their actual oxidation state. For example, the previously mentioned silver incorporation through copolymerization by Chen et al. has shown incorporation of an $\mathrm{Ag}^{+}$-complex into the $\mathrm{CN}$ matrix, but XPS measurements indicate $\mathrm{Ag}(0)$ to $\mathrm{Ag}^{+}$atomic ratios from $61 \%$ to $39 \% .^{246}$ To successfully incorporate metal atoms, the CN matrix may be altered, as exemplified by Pérez-Ramírez and co-workers, who hosted Pd in CN by forming phosphorous doping ( $\mathrm{P}$ was better than other heteroatoms due to increased electron density). ${ }^{657}$ Recent theoretical calculations for different transition metals on the surface of $\mathrm{CN}$ for the electrocatalytic nitrogen reduction reaction (NRR) were performed by Zhang et al.; they predicted that Mo and $\mathrm{V}$ would be the best non-precious metal atomic dopant based on the nitrogen adsorption geometry (standingon and lying-on, respectively). ${ }^{661}$ Chen et al. have also performed a calculational screening, reaching the conclusion that a W atom supported over a single CN layer would be a better catalyst than a stepped $\mathrm{Ru}(0001)$ surface, which has the best activity towards the NRR amongst bulk single-metal catalysts. ${ }^{\mathbf{6 6 2}}$

Already, in the first reports of the photocatalytic activity of CN published a decade ago, two distinct approaches were demonstrated by Wang and co-workers: in the first, Pt NPs were photodeposited on $\mathrm{CN}$ to improve the HER. ${ }^{96}$ In this synthetic mechanism, photoexcited electrons reduce a $\mathrm{Pt}$ source in the surrounding solution, forming a CN/Pt NP HC; in the second, a photocatalytic activity enhancement is achieved through metal doping, an approach that was discussed in Section 4.2.1. In this approach (for example, $\mathrm{Fe}$ (III) species within a $\mathrm{CN}$ matrix ${ }^{663}$ ), metal-nitrogen bonds at the surface are responsible for the catalytic activity. The latter approach can be extended from metal-ion-containing motifs to metal-based materials. 
Zhang et al. have reported using a core@shell Ni@NiO/CN hybrid for the HER. ${ }^{664}$ Liao et al. have used a simple in situ thermal decomposition in air of a Ni(II) precursor physically adsorbed from a solution on $\mathrm{CN}$ to achieve a uniform distribution of NiO NPs throughout CN sheets. This hybrid material exhibits high OER activity due to the formation of a conductive interface and the existence of $\mathrm{Ni}-\mathrm{N}$ bonds at the interface, which are responsible for charge accumulation on the $\mathrm{Ni}$ atom, lowering the adsorption free energy of the OER intermediates. ${ }^{\mathbf{6 6 5}}$ This is an example of how a doping-based catalytic mechanism can exist in CN/NP HCs.

The photodeposition of metals is very common, as mentioned for the case of Pt: when a solution with a metal complex is illuminated in the presence of $\mathrm{CN}$, the photogenerated electrons reduce the metal on its surface, typically forming metal NPs on the CN's surface (as $\mathrm{Cu}^{636}$ ). Recently, Wang et al. have shown that gold can not only be photodeposited, but can also be deposited via piezo-catalytic electron excitation. ${ }^{666}$ This mechanism allows the excitation of electrons following an external ultrasonic stimulus; the excited electrons can be used for $\mathrm{H}_{2} \mathrm{O}_{2}$ generation, and this effect is stronger when the $\mathrm{CN}$ has fewer vacancies.

Other ways of forming metal/CN HCs include reduction of the metal by combination of a reducing agent and high temperature (e.g., refluxing $\mathrm{CN}$ with $\mathrm{RuCl}_{3}$ and ascorbic acid). ${ }^{667}$ or thermal reduction with $\mathrm{H}_{2}(\mathrm{~g})$ after a wet impregnation with a metal salt, as in the case of the $\mathrm{Ni}^{0} \mathrm{NP} / \mathrm{CN} \mathrm{HC}$, which was reported for successful photocatalytic $\mathrm{CO}_{2}$ methanation (Sabatier reaction), ${ }^{668}$ in part due to better charge transfer in the hybrid.

The HC combinations are not limited to single junctions, and the formation of multicomponent structures shows great promise, which augment the HC with high $S_{\mathrm{A}}$, charge separation, catalytically active sites and so forth. For example, ternary $\mathrm{CN} / \mathrm{NiCoP}_{2} \mathrm{MOF} /$ porous carbon HCs were demonstrated for the HER; they take advantage of an improved charge separation at the metal-phosphide/CN interface, the low overpotential of the $\mathrm{NiCoP}_{2}$ cocatalyst, the support functionality of the porous MOF and the improved conductivity of the carbon mediator. ${ }^{669}$ Other examples include combination of a metal/SC junction in addition to a SC/SC junction (e.g., $\mathrm{CuInS}_{2} / \mathrm{Au} / \mathrm{CN},{ }^{670}$ which is useful amongst other applications for the previously discussed Zscheme photocatalysts) or to a SC/enzyme. ${ }^{671}$

\section{Deposition on substrates}

One of the main obstacles for the widespread application of $\mathrm{CN}$ is the difficulty in depositing $\mathrm{CN}$ materials on electrodes while at the same time maintaining their advantageous properties developed in powder. A successful $\mathrm{CN}$ or $\mathrm{CN}$-based hybrid composite on a substrate would continue fulfilling its function under operating conditions (in acidic/alkaline environments, under illumination, under applied voltage bias, etc.). Because of the high importance of this challenging mission, much research has been dedicated towards such an achievement. Our group and others (e.g., Mat Teridi, Zhang, Xue and Pang, Yang, Bian and their co-workers) have recently published review papers discussing deposition techniques and properties of $\mathrm{CN}$ films for photoelectrochemical (PEC) and related applications, such as electrocatalysts, photovoltaics, and energy storage. ${ }^{135,136,672-675}$ For this reason, we will focus only on the most recent progress and refer the reader to the abovementioned reviews for further information regarding performance, mechanisms, and application-specific prospects.

When discussing deposition techniques, the main distinction is whether the $\mathrm{CN}$ is formed on the substrate itself or first synthesized ex situ and then deposited using a given casting technique. Generally, the latter results in an inferior electrical connection and poor mechanical stability due to uncontrolled adhesion of exfoliated CN onto substrates. A good demonstration of this fact is the comparison Ruan et al. performed for $\mathrm{CN}$ on fluorine-doped tin oxide (FTO), where a bulk CN film (obtained by ultrasonication in water and IPA, followed by dropcasting a CN suspension in Nafion resin) was compared to a compact and dense film prepared by other methods (discussed later) and was shown to display inferior crystallinity and performance. ${ }^{676}$ For this reason, high-performance $\mathrm{CN}$ films are usually not prepared using this method. Nevertheless, advances in the field of functionalization and heterojunction formation can still be successfully measured (recent examples are the studies of Zhang and co-workers who formed a $\mathrm{CN} / \mathrm{Cu}$ containing complex hybrid via ball milling and tested it for IR biosensing; ${ }^{677}$ another is the solar aqueous battery ${ }^{678}$ demonstrated by Lotsch and co-workers). Furthermore, the deposition of ex situ synthesized material by casting can be used for the facile electrochemical test of changes of $\mathrm{CN}$ properties (e.g., the influence of exposure to $\gamma$-radiation). ${ }^{679}$ Exceptions include physical deposition techniques, where a simple precursor dispersion is needed (spray coating composites ${ }^{\mathbf{6 8 0}}$ and electrospinning $^{\mathbf{6 8 1}}$ are representative examples).

Some recent progress of note was achieved by the (photo) functionalization of $\mathrm{CN}$ sheets, improving their dispersibility in organic solvents for further spray coating ${ }^{682}$ (opening the exciting practical path of inkjet printing) and casting. The latter was used to fabricate thermoset $\mathrm{CN}$-in-polyester films ${ }^{558}$ and also to incorporate a thin $\mathrm{CN}$ layer as an electron transporting layer in an inverted perovskite solar cell by spin-casting. ${ }^{63}$ Related reports of $\mathrm{CN}$ incorporation described treatment with a functionalizing acid ${ }^{684}$ and iodine doping, ${ }^{685}$ but in these cases the $\mathrm{CN}$ was cast as an additive and not as a standalone film. Similarly, CN was blended with mesoporous $\mathrm{TiO}_{2}$ in a polymeric matrix (polyvinylidene fluoride and $N$-phenethyl-4-piperidone), which was then doctor-bladed. ${ }^{686}$ For further insight, we refer the reader to a recent review by Schmidt and co-workers on the combination of $\mathrm{CN}$ with polymers. ${ }^{67}$ Our group has also recently demonstrated that covalent modification of $\mathrm{CN}$ precursors with polycyclic aromatic hydrocarbons (PAH, such as anthracene and pyrene) amongst other advantages also improves the casting (i.e., drop-casting or spin-coating) of the resulting $\mathrm{CNs}$ (after calcination) on both rigid and flexible substrates, thereby allowing the use of such materials in applications such as electropolymerization and photoanodes in PECs. ${ }^{688}$ 
The 'direct' growth methods of $\mathrm{CN}$ on electrodes include variations of gas-phase and liquid-phase syntheses: ${ }^{135}$ the thermal vapor of various subliming/evaporating $\mathrm{CN}$ precursors can condense on the surface of the substrate or nucleate on preformed 'seeds'; liquid-phase methods rely on the immersion of the substrate into liquid media which include molten $\mathrm{CN}$ precursors, other molten phases (e.g., sulfur ${ }^{689}$ ) and solvothermal solutions. ${ }^{690,691}$ An intermediate situation occurs when a CN-precursor layer is in intimate contact with the substrate and is being thermally condensed, with (strong) CNsubstrate interactions present, as is the case of soaking precursors $^{692}$ and for the method reported by our group, where a paste of supramolecular precursors is doctor-bladed on a substrate prior to its thermal condensation at elevated temperatures in a closed (not sealed) tube. ${ }^{494,693,694}$

The facility and scalability of the doctor-blade method along with the benefits of using supramolecular aggregates (e.g., precise control over composition) as precursors are its most appealing features. Furthermore, it allows the incorporation of conductive elements, such as rGO, to improve the electron diffusion length within the film (up to $c a .36 \mu \mathrm{m}$ ).${ }^{693}$ We have found that to improve the packing and order of the $\mathrm{CN}$ layer (motivated by Durrant and co-workers' report on electron trap states at the surface ${ }^{695}$ and the electron transport properties of crystalline CN. See discussion in Section 4.4) it is better to use a molecular precursor rather than a supramolecular precursor. To this end, we have dipped the substrate into a hot supersaturated melamine solution to deposit a melamine film, which after calcination formed a crystalline $\mathrm{CN}$ layer. ${ }^{696}$ Melamine alone cannot be doctor-bladed, but upon addition of GO, a viscous powder is formed, which allows the deposition. ${ }^{\mathbf{9 9 4}}$

Recent advances achieved using vapor-based methods include multi-step deposition resulting in pinhole-free layers, ${ }^{\mathbf{6 9} 7}$ heteroatom-incorporation ${ }^{698}$ and heterojunctions of $\mathrm{CN}$ containing different heteroatoms, ${ }^{699}$ metal-ion incorporation ${ }^{700}$ and control over crystallinity in $\sim 0.2 \mu \mathrm{m}$-thick formaldehyde-doped CN layers. $^{701}$

It is important to note that the discussion in this section focused on the deposition of thin $\mathrm{CN}$ layers over substrates with relatively low roughness (as is the case for the standard transparent conductive oxides (TCOs), which are coated on rigid glass or flexible polymer-based substrates in photoelectronic devices thanks to their combination of electronic conductance and optical transparency). However, the same principles can be applied to interface $\mathrm{CN}$ with different (porous) materials, such as carbon-based materials, membranes (serving as a hard template), ${ }^{\mathbf{7 0 2 , 7 0 3}}$ inorganic nanostructures, ${ }^{\mathbf{6 2 0 , 6 9 2 , 7 0 4}}$ etc. All the above-mentioned approaches can be used for the formation of semiconductor heterojunctions ${ }^{\mathbf{7 0 4 , 7 0 5}}$ between $\mathrm{CN}$ and other materials (to improve photoelectrode performance, for example, by improving light harvesting, charge separation and charge carrier migration paths). An elegant demonstration of a complex HC for PECs was shown by Qin et al., who thermally polymerized $\mathrm{CN}$ over a substrate with vertically aligned $\mathrm{TiO}_{2}$ nanorods, followed by metal ion soaking and gas-phase phosphidation, which both dopes the $\mathrm{CN}$ with $\mathrm{P}$ and forms metal (M
$=\mathrm{Fe}, \mathrm{Cu}$ ) phosphide oxidation catalysts. This synthesis yields FTO/TiO ${ }_{2} /\left(\right.$ P-doped)CN/M $\mathrm{P} \mathrm{HC}$ photoanodes. ${ }^{706}$

For some applications, such as photocatalysis, free-standing films are of value. To this end, on top of common synthetic pathways such as filtration or freeze-drying, ${ }^{707}$ the deposition on substrates can be used as a first step before peeling-off ${ }^{\mathbf{7 0 0}, 708}$ or before the transformation of the substrate itself into an electrode, as our group has demonstrated by the calcination of a filter paper coated with $\mathrm{CN}$ precursors, transforming it into a free-standing $\mathrm{C} / \mathrm{CN}$ hybrid. ${ }^{709}$

Significant progress is underway in the application of $\mathrm{CN}$ as a photoelectrode in the emerging organic PEC devices ${ }^{\mathbf{7 1 0}}$ or in hybrid scenarios where $\mathrm{CN}$ complements the better-established inorganic layers, ${ }^{711}$ with possible metal-based cocatalysts. ${ }^{712}$ In addition, the ability to deposit large-scale porous films ${ }^{713}$ of $\mathrm{CN}$ paves the way also for its application as a particulate photocatalyst, ${ }^{714}$ i.e., a film of photoactive material (composite) that can perform energy conversion reactions, such as hydrogen production, without connection to an external electric bias, as demonstrated by the Domen group for other materials. ${ }^{715-717}$

\section{Prospects and concluding remarks}

In this review, we summarized the recent developments in the synthesis, growth methods and applications of carbon nitridebased materials, ranging from carbons, N-doped carbons, $\mathrm{C}_{2} \mathrm{~N}, \mathrm{C}_{3} \mathrm{~N}_{4}$ to other $\mathrm{C}-\mathrm{N}$ containing polymers. We have mainly focused on melon carbon nitrides (commonly referred to in the literature as $\mathrm{C}_{3} \mathrm{~N}_{4}$ ) owing to their attractive properties, e.g., tunable band gap, low-price, stability under harsh conditions and suitable energy band positions, enabling various reduction and oxidation reactions along with a wide range of applications.

The exploration and development of synthetic routes, characterization techniques and fundamental understanding of structural features play an essential role in opening the path towards the utilization of these materials in practical applications. We discussed the synthetic methods in detail, including templating and doping techniques, as well as emerging approaches, such as the use of supramolecular chemistry and covalent functionalization, with a special emphasis on the rational design of a modified-CN with controlled chemical, photophysical and catalytic properties. The methods to affect and study their electronic and optical properties and their photo-, electro- and photoelectro-catalytic performance for various energy-related applications were elaborated upon. The manipulation of the electronic and catalytic properties by interfacing with other materials, by single atom doping and by molecular modification was shown. A special emphasis was placed on the correlation between their structural properties and their activity in a given reaction. In the last part, the recent progress in the growth and deposition of carbon nitride materials on surfaces and their utilization as photoelectrodes were briefly introduced, with a focus on the deposition techniques and the subsequent use of $\mathrm{CN}$ films as photoelectrodes for water splitting. Based on the current status and challenges in the synthesis and incorporation of (modified) $\mathrm{CN}$ materials into 
practical devices, in our opinion, future research should pay attention to the following:

1. The synthetic paths should be further improved, to allow a better design of the final properties, such as the specific surface area, morphology, optical band gap, crystallinity and reaction yield. Moreover, the development of alternative synthetic methods (low temperature reaction, solvothermal, etc.) may lead to the reduction of the costs inherent to the required high-temperature synthesis.

2. A thorough evaluation of the chemical processes occurring during thermal condensation by in situ techniques and operando methods would improve both mechanistic understanding and reproducibility. Specifically, spectroscopic means coupled to thermogravimetric analysis or electrochemical means, in situ heating electronic microscopy and other techniques could bring about a deeper understanding of the chemical, electronic and morphological transformations occurring during the thermal condensation and polymerization, which form $\mathrm{C}_{x} \mathrm{~N}_{y}\left(\mathrm{H}_{z}\right)$ polymers.

3. The understanding of the photophysical properties, i.e., charge separation and transfer properties as well as energy band structure is crucial for the further integration of CN materials as practical absorbers and catalysts.

4. For photodriven reactions, the effective optical band gap should be reduced to about $2 \mathrm{eV}$ to permit an effective utilization of the solar spectrum and achieve higher overall efficiencies.

5. We envision the emergence of $\mathrm{CN}$ materials in related research fields, such as solar cells, photoelectrochemical cells, imaging, biomedical and others, especially in conjugation with other organic and inorganic materials as hybrid nanostructures and composites, where control over the interface morphology and defects has room for further improvement without increasing complexity.

6. For practical applications, the scalability of the preparation protocols and the long-term stability under operational conditions will define the real-world feasibility of the material utilization in a given application.

All these efforts together with the substantial ongoing progress in the field will make $\mathrm{CN}$ materials competitive candidate semiconductors for many energy-related applications and other fields spanning photo- and electro-catalysis, organic transformation, sensors and more in the future.

\section{Conflicts of interest}

The authors declare no conflicts of interest.

\section{Abbreviations}

$\begin{array}{ll}\text { 2D } & \text { Two-dimensional } \\ \text { AQY } & \text { Average quantum yield } \\ \text { BET } & \text { Brunauer-Emmett-Teller model } \\ \text { CB } & \text { Conduction band } \\ \text { DFT } & \text { Density functional theory } \\ \mathrm{e}^{-} & \text {Electron (negative charge carrier) }\end{array}$

$E_{\mathrm{F}} \quad$ Fermi level (Fermi energy)

$E_{\mathrm{g}} \quad$ Band gap (electronic)

EDS Energy-dispersive X-ray spectroscopy

EELS Electron energy loss spectroscopy

EPR Electron paramagnetic resonance

FTIR Fourier-transform infrared spectroscopy

$\mathrm{h}^{+} \quad$ Hole (positive charge carrier)

HC Hybrid composite

HER Hydrogen evolution reaction

HOMO Highest occupied molecular orbital

LUMO Lowest unoccupied molecular orbital

MOF Metal-organic framework

NMR Nuclear magnetic resonance spectroscopy

NP Nanoparticle

NRR Nitrogen reduction reaction

OER Oxygen evolution reaction

QD Quantum dot

ROS Reactive oxygen species

$S_{\mathrm{A}} \quad$ Specific surface area

SC Semiconductor

TEM Transmission electron microscopy

TCO Transparent conductive oxide (e.g., ITO, FTO, and AZO)

TOF Turnover frequency

vs. Versus

VB Valance band

vdW van der Waals

XPS X-ray photoelectron spectroscopy

XRD X-ray diffraction

\section{Chemicals, materials and solvents}

$\mathrm{AAO}$

acac

BA

$\mathrm{BN}$

BCN

BPA

CY

CA

$\mathrm{CN}$

$\mathrm{CN}-\mathrm{H}$

CNQDS

$\mathrm{CM}$

$\mathrm{CMB}$

$\mathrm{COF}$

DCDA

DMF

DMSO

DPT

EDA

FTO

HRP

IPA

MB

MO

NIPA

NMP
Anodic aluminum oxide

Acetylacetonate

Barbituric acid

Boron nitride

Boron-doped carbon nitride

Bisphenol A

Cyanamide

Cyanuric acid

Carbon nitride polymers

Carbon nitride-based hydrogel

Carbon nitride quantum dots

Cyanuric acid-melamine

Cyanuric acid-melamine-barbituric acid

Covalent-organic framework

Dicyandiamide

$N, N$-Dimethylformamide

Dimethylsulfoxide

2,4-Diamino-6-phenyl-1,3,5-triazine

Ethylenediamine

Fluorine-doped tin oxide

Horseradish peroxidase

Isopropyl alcohol (2-propanol)

Methylene blue dye

Methyl orange

Poly( $N$-isopropylacrylamide)

$N$-Methylpyrrolidone 


$\begin{array}{ll}\text { PAH } & \text { Polycyclic aromatic hydrocarbon } \\ \text { PCN } & \text { Phosphorous-doped carbon nitride } \\ \text { PCT } & \text { Phosphonitrilic chloride trimer } \\ \text { PHTIK } & \text { Potassium poly(heptazine imide) } \\ \text { PMDA } & \text { 1,2,4,5-Benzene tetracarboxylic dianhydride } \\ \text { PTI } & \text { Poly(triazine imide) } \\ \text { rGO } & \text { Reduced graphene oxide } \\ \text { RhB } & \text { Rhodamine B dye } \\ \text { SBA-15 } & \text { Mesoporous silica nanoparticles } \\ \text { SCN } & \text { Sulfur-doped carbon nitride } \\ \text { TEOA } & \text { Triethanolamine } \\ \text { THDT } & \text { 2,4,6-Trihydrazino-1,3,5-triazine } \\ \text { TMB } & \text { Tetramethylbenzidine }\end{array}$

\section{Acknowledgements}

We acknowledge the financial support of the Israel Science Foundation (ISF), grant no. 1161/17 and the Minerva Center No. 117873. This project has received funding from the European Research Council (ERC) under the European Union's Horizon 2020 research and innovation program (grant agreement no. [849068]).

\section{References}

1 A. K. Geim and K. S. Novoselov, Nat. Mater., 2007, 6, 183191.

2 T. W. Ebbesen and P. M. Ajayan, Nature, 1992, 358, 220-222.

3 R. Taylor and D. R. M. Walton, Nature, 1993, 363, 685-693.

4 M. Antonietti, N. Fechler and T.-P. Fellinger, Chem. Mater., 2013, 26, 196-210.

5 M. Oschatz and M. Antonietti, Energy Environ. Sci., 2018, 11, 57-70.

6 C. Hu, Y. Lin, J. W. Connell, H.-M. Cheng, Y. Gogotsi, M.-M. Titirici and L. Dai, Adv. Mater., 2019, 31, 1806128.

7 M.-M. Titirici, R. J. White, N. Brun, V. L. Budarin, D. S. Su, F. del Monte, J. H. Clark and M. J. MacLachlan, Chem. Soc. Rev., 2015, 44, 250-290.

8 M. Antonietti, N. Lopez-Salas and A. Primo, Adv. Mater., 2019, 31, 1805719.

9 Z. Xu, J. Park, G. Yoon, H. Kim and K. Kang, Small Methods, 2019, 3, 1800227.

10 K. Tang, L. Fu, R. J. White, L. Yu, M.-M. Titirici, M. Antonietti and J. Maier, Adv. Energy Mater., 2012, 2, 873-877.

11 G. A. Tiruye, D. Muñoz-Torrero, T. Berthold, J. Palma, M. Antonietti, N. Fechler and R. Marcilla, J. Mater. Chem. A, 2017, 5, 16263-16272.

12 R. Daiyan, X. Tan, R. Chen, W. H. Saputera, H. A. Tahini, E. Lovell, Y. H. Ng, S. C. Smith, L. Dai, X. Lu and R. Amal, ACS Energy Lett., 2018, 3, 2292-2298.

13 A. B. Jorge, R. Jervis, A. P. Periasamy, M. Qiao, J. Feng, L. N. Tran and M.-M. Titirici, Adv. Energy Mater., 2020, 10, 1902494.
14 C. Tang, H.-F. Wang, X. Chen, B.-Q. Li, T.-Z. Hou, B. Zhang, Q. Zhang, M.-M. Titirici and F. Wei, Adv. Mater., 2016, 28, 6845-6851.

15 J. Kong, A. M. Cassell and H. Dai, Chem. Phys. Lett., 1998, 292, 567-574.

16 A. Reina, X. Jia, J. Ho, D. Nezich, H. Son, V. Bulovic, M. S. Dresselhaus and J. Kong, Nano Lett., 2008, 9, 30-35.

17 L. Cai and G. Yu, Small Methods, 2019, 1900071.

18 C. Lavorato, A. Primo, R. Molinari and H. García, ACS Catal., 2014, 4, 497-504.

19 J. Xu, J. Zhu, X. Yang, S. Cao, J. Yu, M. Shalom and M. Antonietti, Adv. Mater., 2016, 28, 6727-6733.

20 L. Zhao, N. Baccile, S. Gross, Y. Zhang, W. Wei, Y. Sun, M. Antonietti and M.-M. Titirici, Carbon, 2010, 48, 37783787.

21 B. Hu, K. Wang, L. Wu, S.-H. Yu, M. Antonietti and M.-M. Titirici, Adv. Mater., 2010, 22, 813-828.

22 M.-M. Titirici and M. Antonietti, Chem. Soc. Rev., 2010, 39, 103-116.

23 L. K. Gil-Herrera, Á. Blanco, B. H. Juárez and C. López, Small, 2016, 12, 4357-4362.

24 M. Sevilla and A. B. Fuertes, Carbon, 2009, 47, 2281-2289.

25 M. Sevilla and A. B. Fuertes, Chem.-Eur. J., 2009, 15, 41954203.

26 Y. Chen, X. Zhang and Z. Zhou, Small Methods, 2019, 3, 1900050.

27 N. Fechler and M. Antonietti, Nano Today, 2015, 10, 593614.

28 R. Yan, T. Heil, V. Presser, R. Walczak, M. Antonietti and M. Oschatz, Adv. Sustainable Syst., 2018, 2, 1700128.

29 J. P. Paraknowitsch and A. Thomas, Energy Environ. Sci., 2013, 6, 2839-2855.

30 M. Antonietti and M. Oschatz, Adv. Mater., 2018, 30, 1706836

31 L. K. Gil-Herrera, J. A. Pariente, F. Gallego-Gómez, F. Gándara, B. H. Juárez, Á. Blanco and C. López, Adv. Funct. Mater., 2018, 28, 1703885.

32 H.-L. Jiang, B. Liu, Y.-Q. Lan, K. Kuratani, T. Akita, H. Shioyama, F. Zong and Q. Xu, J. Am. Chem. Soc., 2011, 133, 11854-11857.

33 W. Chen, M. Wan, Q. Liu, X. Xiong, F. Yu and Y. Huang, Small Methods, 2019, 3, 1800323.

34 H. Zhu, J. Yin, X. Wang, H. Wang and X. Yang, Adv. Funct. Mater., 2013, 23, 1305-1312.

35 L.-F. Chen, Z.-H. Huang, H.-W. Liang, H.-L. Gao and S.-H. Yu, Adv. Funct. Mater., 2014, 24, 5104-5111.

36 N. Fechler, T.-P. Fellinger and M. Antonietti, J. Mater. Chem. A, 2013, 1, 14097-14102.

37 D. Kim, O. L. Li and N. Saito, Phys. Chem. Chem. Phys., 2015, 17, 407-413.

38 Y.-Q. Zhao, M. Lu, P.-Y. Tao, Y.-J. Zhang, X.-T. Gong, Z. Yang, G.-Q. Zhang and H.-L. Li, J. Power Sources, 2016, 307, 391-400.

39 S.-A. Wohlgemuth, F. Vilela, M.-M. Titirici and M. Antonietti, Green Chem., 2012, 14, 741-749. 
40 S.-A. Wohlgemuth, R. J. White, M.-G. Willinger, M.-M. Titirici and M. Antonietti, Green Chem., 2012, 14, 1515-1523.

41 L. Xia, X. Wu, Y. Wang, Z. Niu, Q. Liu, T. Li, X. Shi, A. M. Asiri and X. Sun, Small Methods, 2019, 3, 1800251.

42 J. Zhang and L. Dai, ACS Catal., 2015, 5, 7244-7253.

43 L. Zhang, Y. Wang, Z. Niu and J. Chen, Small Methods, 2019, 1800443.

44 X. Yu, P. Han, Z. Wei, L. Huang, Z. Gu, S. Peng, J. Ma and G. Zheng, Joule, 2018, 2, 1610-1622.

45 R. Lv, G. Chen, Q. Li, A. McCreary, A. Botello-Méndez, S. V. Morozov, L. Liang, X. Declerck, N. Perea-López, D. A. Cullen, S. Feng, A. L. Elías, R. Cruz-Silva, K. Fujisawa, M. Endo, F. Kang, J.-C. Charlier, V. Meunier, M. Pan, A. R. Harutyunyan, K. S. Novoselov and M. Terrones, Proc. Natl. Acad. Sci., 2015, 112, 14527-14532. 46 J. Yi, Y. Qing, C. Wu, Y. Zeng, Y. Wu, X. Lu and Y. Tong, J. Power Sources, 2017, 351, 130-137.

47 J. Wu, C. Jin, Z. Yang, J. Tian and R. Yang, Carbon, 2015, 82, 562-571.

48 M.-S. Balogun, W. Qiu, Y. Huang, H. Yang, R. Xu, W. Zhao, G.-R. Li, H. Ji and Y. Tong, Adv. Mater., 2017, 29, 1702095.

49 X. Gong, S. Liu, C. Ouyang, P. Strasser and R. Yang, ACS Catal., 2015, 5, 920-927.

50 J. Patiño, N. López-Salas, M. C. Gutiérrez, D. Carriazo, M. L. Ferrer and F. del Monte, J. Mater. Chem. A, 2016, 4, 1251-1263.

51 Y. Wen, B. Wang, C. Huang, L. Wang and D. HulicovaJurcakova, Chem.-Eur. J., 2015, 21, 80-85.

52 J. Tzadikov, M. Auinat, J. Barrio, M. Volokh, G. Peng, C. Gervais, Y. Ein-Eli and M. Shalom, ChemSusChem, 2018, 11, 2912-2920.

53 J. Tzadikov, M. Amsellem, H. Amlani, J. Barrio, A. Azoulay, M. Volokh, S. Kozuch and M. Shalom, Angew. Chem., Int. Ed., 2019, 58, 14964-14968.

54 A. Zahoor, M. Christy, Y. J. Hwang, Y. R. Lim, P. Kim and K. S. Nahm, Appl. Catal., B, 2014, 147, 633-641.

55 D. Guo, R. Shibuya, C. Akiba, S. Saji, T. Kondo and J. Nakamura, Science, 2016, 351, 361-365.

56 K. Sakaushi and M. Antonietti, Acc. Chem. Res., 2015, 48, 1591-1600.

57 W. Wang and A. D. Schlüter, Macromol. Rapid Commun., 2019, 40, 1800719.

58 H. Furukawa and O. M. Yaghi, J. Am. Chem. Soc., 2009, 131, 8875-8883.

59 L. Merí-Bofí, S. Royuela, F. Zamora, M. L. Ruiz-González, J. L. Segura, R. Muñoz-Olivas and M. J. Mancheño, J. Mater. Chem. A, 2017, 5, 17973-17981.

60 D. Rodríguez-San-Miguel and F. Zamora, Chem. Soc. Rev., 2019, 48, 4375-4386.

61 Y. Zeng, R. Zou and Y. Zhao, Adv. Mater., 2016, 28, 28552873.

62 V. S. Vyas, F. Haase, L. Stegbauer, G. Savasci, F. Podjaski, C. Ochsenfeld and B. V Lotsch, Nat. Commun., 2015, 6, 8508.

63 J. Romero, D. Rodriguez-San-Miguel, A. Ribera, R. MasBallesté, T. F. Otero, I. Manet, F. Licio, G. Abellán,
F. Zamora and E. Coronado, J. Mater. Chem. A, 2017, 5, 4343-4351.

64 X. Liu, L. Zhou, Y. Zhao, L. Bian, X. Feng and Q. Pu, ACS Appl. Mater. Interfaces, 2013, 5, 10280-10287.

65 Z. Xiang, D. Cao, L. Huang, J. Shui, M. Wang and L. Dai, Adv. Mater., 2014, 26, 3315-3320.

66 K. Shen, X. Chen, J. Chen and Y. Li, ACS Catal., 2016, 6, 5887-5903.

67 R. Walczak, B. Kurpil, A. Savateev, T. Heil, J. Schmidt, Q. Qin, M. Antonietti and M. Oschatz, Angew. Chem., Int. Ed., 2018, 57, 10765-10770.

68 C. Van Nguyen, S. Lee, Y. G. Chung, W.-H. Chiang and K. C.-W. Wu, Appl. Catal., B, 2019, 257, 117888.

69 A. Aijaz, N. Fujiwara and Q. Xu, J. Am. Chem. Soc., 2014, 136, 6790-6793.

70 J. Hwang, R. Walczak, M. Oschatz, N. V Tarakina and B. V. K. J. Schmidt, Small, 2019, 15, 1901986.

71 T. Jordan, M. Shalom, M. Antonietti and N. Fechler, AsiaPacific J. Chem. Eng., 2016, 11, 866-873.

72 Z. Zhou, F. He, Y. Shen, X. Chen, Y. Yang, S. Liu, T. Mori and Y. Zhang, Chem. Commun., 2017, 53, 2044-2047.

73 X. Feng, L. Bian, J. Ma, L. Zhou, X. Wang, G. Guo and Q. Pu, Chem. Commun., 2019, 55, 3963-3966.

74 J. Kouvetakis, R. B. Kaner, M. L. Sattler and N. Bartlett, J. Chem. Soc., Chem. Commun., 1986, 1758-1759.

75 J. Mahmood, E. K. Lee, M. Jung, D. Shin, I.-Y. Jeon, S.-M. Jung, H.-J. Choi, J.-M. Seo, S.-Y. Bae, S.-D. Sohn, N. Park, J. H. Oh, H.-J. Shin and J.-B. Baek, Nat. Commun., 2015, 6, 6486.

76 J. Mahmood, E. K. Lee, M. Jung, D. Shin, H.-J. Choi, J.-M. Seo, S.-M. Jung, D. Kim, F. Li, M. S. Lah, N. Park, H.-J. Shin, J. H. Oh and J.-B. Baek, Proc. Natl. Acad. Sci. U. S. A., 2016, 113, 7414-7419.

77 M. R. A. Kishore and P. Ravindran, ChemPhysChem, 2017, 18, 1526-1532.

78 S. Yang, W. Li, C. Ye, G. Wang, H. Tian, C. Zhu, P. He, G. Ding, X. Xie, Y. Liu, Y. Lifshitz, S.-T. Lee, Z. Kang and M. Jiang, Adv. Mater., 2017, 29, 1605625.

79 J. Xu, J. Mahmood, Y. Dou, S. Dou, F. Li, L. Dai and J.-B. Baek, Adv. Mater., 2017, 29, 1702007.

80 F. Opoku, K. K. Govender, C. G. C. E. van Sittert and P. P. Govender, Adv. Sustainable Syst., 2017, 1, 1700006.

81 X. Chen, S. Shen, L. Guo and S. S. Mao, Chem. Rev., 2010, 110, 6503-6570.

82 Z. Ma, X. Zhao, Q. Tang and Z. Zhou, Int. J. Hydrogen Energy, 2014, 39, 5037-5042.

83 Q. Wang, H. Gou, L. Zhu, H.-T. Huang, A. Biswas, B. L. Chaloux, A. Epshteyn, J. P. Yesinowski, Z. Liu, G. Cody, M. Ma, Z. Zhao, Y. Fei, C. Prescher, E. Greenberg, V. B. Prakapenka and T. A. Strobel, ACS Mater. Lett., 2019, 1, 14-19.

84 J. Barrio, N. Karjule, J. Qin and M. Shalom, ChemCatChem, 2019, 11, 6295-6300.

85 J. Liebig, Justus Liebigs Ann. Chem., 1844, 50, 337-363. 86 J. Liebig, Justus Liebigs Ann. Chem., 1855, 95, 257-282.

87 H. Montigaud, B. Tanguy, G. Demazeau, I. Alves and S. Courjault, J. Mater. Sci., 2000, 35, 2547-2552. 
88 E. Kroke and M. Schwarz, Coord. Chem. Rev., 2004, 248, 493-532.

89 A. Y. Liu and M. L. Cohen, Phys. Rev. B: Solid State, 1990, 41, 10727-10734.

90 A. Y. Liu and M. L. Cohen, Science, 1989, 245, 841-842.

91 C. Niu, Y. Z. Lu and C. M. Lieber, Science, 1993, 261, 334337.

92 P. Kumar, E. Vahidzadeh, U. K. Thakur, P. Kar, K. M. Alam, A. Goswami, N. Mahdi, K. Cui, G. M. Bernard, V. K. Michaelis and K. Shankar, J. Am. Chem. Soc., 2019, 141, 5415-5436.

93 I. Y. Kim, S. Kim, S. Premkumar, J.-H. Yang, S. Umapathy and A. Vinu, Small, 2019, n/a, 1903572.

94 J. Zhang, Y. Zhao, Z. Wang, G. Yang, J. Tian, D. Ma and Y. Wang, New J. Chem., 2020, 44, 422-427.

95 T. S. Miller, A. B. Jorge, T. M. Suter, A. Sella, F. Corà and P. F. McMillan, Phys. Chem. Chem. Phys., 2017, 19, 1561315638.

96 X. Wang, K. Maeda, A. Thomas, K. Takanabe, G. Xin, J. M. Carlsson, K. Domen and M. Antonietti, Nat. Mater., 2009, 8, 76-80.

97 D. Ni, Y. Zhang, Y. Shen, S. Liu and Y. Zhang, Chin. Chem. Lett., 2020, 31, 115-118.

98 R. Schlo, A. Thomas, A. Fischer, F. Goettmann, M. Antonietti, J. Mu and J. M. Carlsson, J. Mater. Chem., 2008, 4893-4908.

99 B. Zhu, B. Cheng, L. Zhang and J. Yu, Carbon Energy, 2019, 1, 32-56.

100 W. Chao, F. Huiqing, R. Xiaohu, M. Jiangwei, F. Jiawen and W. Weijia, ChemSusChem, 2018, 11, 700-708.

101 X. Li, J. Zhang, L. Shen, Y. Ma, W. Lei, Q. Cui and G. Zou, Appl. Phys. A, 2009, 94, 387-392.

102 S. C. Yan, Z. S. Li and Z. G. Zou, Langmuir, 2009, 25, 1039710401.

103 W. Tu, Y. Xu, J. Wang, B. Zhang, T. Zhou, S. Yin, S. Wu, C. Li, Y. Huang, Y. Zhou, Z. Zou, J. Robertson, M. Kraft and R. Xu, ACS Sustainable Chem. Eng., 2017, 5, 7260-7268.

104 J. Yang, X. Wu, X. Li, Y. Liu, M. Gao, X. Liu, L. Kong and S. Yang, Appl. Phys. A, 2011, 105, 161.

105 H.-S. Zhai, L. Cao and X.-H. Xia, Chin. Chem. Lett., 2013, 24, 103-106.

106 J. Xu, Y. Li, S. Peng, G. Lu and S. Li, Phys. Chem. Chem. Phys., 2013, 15, 7657-7665.

107 Y. Zhang, J. Liu, G. Wu and W. Chen, Nanoscale, 2012, 4, 5300-5303.

108 J. Liu, T. Zhang, Z. Wang, G. Dawson and W. Chen, J. Mater. Chem., 2011, 21, 14398-14401.

109 J. Bai, Q. Han, Z. Cheng and L. Qu, Chem.-Asian J., 2018, 13, 3160-3164.

110 G. Zhang, J. Zhang, M. Zhang and X. Wang, J. Mater. Chem., 2012, 22, 8083-8091.

111 F. Dong, Y. Sun, L. Wu, M. Fu and Z. Wu, Catal. Sci. Technol., 2012, 2, 1332-1335.

112 Y. Hong, E. Liu, J. Shi, X. Lin, L. Sheng, M. Zhang, L. Wang and J. Chen, Int. J. Hydrogen Energy, 2019, 44, 7194-7204.
113 D. Dontsova, S. Pronkin, M. Wehle, Z. Chen, C. Fettkenhauer, G. Clavel and M. Antonietti, Chem. Mater., 2015, 27, 5170-5179.

114 Y. Cui, G. Zhang, Z. Lin and X. Wang, Appl. Catal., B, 2016, 181, 413-419.

115 A. T. Montoya and E. G. Gillan, ACS Appl. Nano Mater., 2018, 1, 5944-5956.

116 A. Savateev, S. Pronkin, J. D. Epping, M. G. Willinger, M. Antonietti and D. Dontsova, J. Mater. Chem. A, 2017, 5, 8394-8401.

117 J. Zhang, J. Sun, K. Maeda, K. Domen, P. Liu, M. Antonietti, X. Fu and X. Wang, Energy Environ. Sci., 2011, 4, 675.

118 R. C. Dante, P. Martín-Ramos, F. M. Sánchez-Arévalo, L. Huerta, M. Bizarro, L. M. Navas-Gracia and J. MartínGil, J. Solid State Chem., 2013, 201, 153-163.

119 B. Long, J. Lin and X. Wang, J. Mater. Chem. A, 2014, 2, 2942-2951.

120 Y. Cui, J. Zhang, G. Zhang, J. Huang, P. Liu, M. Antonietti and X. Wang, J. Mater. Chem., 2011, 21, 13032-13039.

121 Z. Zhang, Y. Zhang, L. Lu, Y. Si, S. Zhang, Y. Chen, K. Dai, P. Duan, L. Duan and J. Liu, Appl. Surf. Sci., 2017, 391, 369375.

122 H.-J. Li, D.-J. Qian and M. Chen, ACS Appl. Mater. Interfaces, 2015, 7, 25162-25170.

123 Y. Zhang, Q. Pan, G. Chai, M. Liang, G. Dong, Q. Zhang and J. Qiu, Sci. Rep., 2013, 3, 1943.

124 H. Zhang and A. Yu, J. Phys. Chem. C, 2014, 118, 1162811635.

125 P. Wu, J. Wang, J. Zhao, L. Guo and F. E. Osterloh, J. Mater. Chem. A, 2014, 2, 20338-20344.

126 H. Lan, L. Li, X. An, F. Liu, C. Chen, H. Liu and J. Qu, Appl. Catal., B, 2017, 204, 49-57.

127 G. Dong, Y. Zhang, Q. Pan and J. Qiu, J. Photochem. Photobiol., C, 2014, 20, 33-50.

128 Y. Zheng, J. Liu, J. J. Liang, M. Jaroniec and S. Z. Qiao, Energy Environ. Sci., 2012, 5, 6717-6731.

129 J. Zhu, P. Xiao, H. Li and A. C. Carabineiro, ACS Appl. Mater. Interfaces, 2014, 6, 16449-16465.

130 C. Shaowen, L. Jingxiang, Y. Jiaguo and J. Mietek, Adv. Mater., 2015, 27, 2150-2176.

131 J. Wen, J. Xie, X. Chen and X. Li, Appl. Surf. Sci., 2017, 391, 72-123.

132 K. S. Lakhi, D.-H. Park, K. Al-Bahily, W. Cha, B. Viswanathan, J.-H. Choy and A. Vinu, Chem. Soc. Rev., 2017, 46, 72-101.

133 W. J. Ong, L. L. Tan, Y. H. Ng, S. T. Yong and S. P. Chai, Chem. Rev., 2016, 116, 7159-7329.

134 S. Sun and S. Liang, Nanoscale, 2017, 9, 10544-10578.

135 M. Volokh, G. Peng, J. Barrio and M. Shalom, Angew. Chem., Int. Ed., 2019, 58, 6138-6151.

136 J. Safaei, N. A. Mohamed, M. F. Mohamad Noh, M. F. Soh, N. A. Ludin, M. A. Ibrahim, W. N. Roslam Wan Isahak and M. A. Mat Teridi, J. Mater. Chem. A, 2018, 6, 22346-22380.

137 S. Kumar, S. Karthikeyan and A. Lee, Catalysts, 2018, 8, 74.

138 L. Wang, Y. Tong, J. Feng, J. Hou, J. Li, X. Hou and J. Liang, Sustainable Mater. Technol., 2019, 19, e00089. 
139 F. K. Kessler, Y. Zheng, D. Schwarz, C. Merschjann, W. Schnick, X. Wang and M. J. Bojdys, Nat. Rev. Mater., 2017, 2, 17030.

140 Y. Yang, S. Wang, Y. Li, J. Wang and L. Wang, Chem.-Asian J., 2017, 12, 1421-1434.

141 A. Naseri, M. Samadi, A. Pourjavadi, A. Z. Moshfegh and S. Ramakrishna, J. Mater. Chem. A, 2017, 5, 23406-23433.

142 J. Yi, W. El-Alami, Y. Song, H. Li, P. M. Ajayan and H. Xu, Chem. Eng. J., 2020, 382, 122812.

143 X. Wang, K. Maeda, X. Chen, K. Takanabe, K. Domen, Y. Hou, X. Fu and M. Antonietti, J. Am. Chem. Soc., 2009, 131, 1680-1681.

144 C. Butchosa, P. Guiglion and M. A. Zwijnenburg, J. Phys. Chem. C, 2014, 118, 24833-24842.

145 Y. Zhu, D. Zhang, L. Gong, L. Zhang and Z. Xia, Front. Mater., 2019, 6, 1-10.

146 G. Liao, Y. Gong, L. Zhang, H. Gao, G.-J. Yang and B. Fang, Energy Environ. Sci., 2019, 12, 2080-2147.

147 S. Patnaik, S. Martha and K. M. Parida, RSC Adv., 2016, 6, 46929-46951.

148 M. Z. Rahman, K. Davey and C. B. Mullins, Adv. Sci., 2018, 5, 1800820.

149 M. S. Nasir, G. Yang, I. Ayub, S. Wang, L. Wang, X. Wang, W. Yan, S. Peng and S. Ramakarishna, Appl. Catal., B, 2019, 257, 117855.

150 Y. Wang, A. Vogel, M. Sachs, R. S. Sprick, L. Wilbraham, S. J. A. Moniz, R. Godin, M. A. Zwijnenburg, J. R. Durrant, A. I. Cooper and J. Tang, Nat. Energy, 2019, 4, 746-760.

151 J. Lin, Z. Pan and X. Wang, ACS Sustainable Chem. Eng., 2014, 2, 353-358.

152 Y. Fang and X. Wang, Chem. Commun., 2018, 54, 5674-5687. 153 S. N. Talapaneni, G. Singh, I. Y. Kim, K. AlBahily, A. H. AlMuhtaseb, A. S. Karakoti, E. Tavakkoli and A. Vinu, Adv. Mater., 2019, 0, 1904635.

154 V. Hasija, P. Raizada, A. Sudhaik, K. Sharma, A. Kumar, P. Singh, S. B. Jonnalagadda and V. K. Thakur, Appl. Mater. Today, 2019, 15, 494-524.

155 R. Hu, X. Wang, S. Dai, D. Shao, T. Hayat and A. Alsaedi, Chem. Eng. J., 2015, 260, 469-477.

156 A. L. Stroyuk, A. E. Raevskaya and S. Y. Kuchmy, Theor. Exp. Chem., 2019, 55, 147-172.

157 J. Liu, H. Wang and M. Antonietti, Chem. Soc. Rev., 2016, 45, 2308-2326.

158 Z. Zhou, Y. Zhang, Y. Shen, S. Liu and Y. Zhang, Chem. Soc. Rev., 2018, 47, 2298-2321.

159 J. Xu, M. Antonietti and M. Shalom, Chem.-Asian J., 2016, 11, 2499-2512.

160 A. B. Jorge, D. J. Martin, M. T. S. Dhanoa, A. S. Rahman, N. Makwana, J. Tang, A. Sella, F. Corà, S. Firth, J. A. Darr and P. F. McMillan, J. Phys. Chem. C, 2013, 117, 7178-7185.

161 K. Maeda, X. Wang, Y. Nishihara, D. Lu, M. Antonietti and K. Domen, J. Phys. Chem. C, 2009, 113, 4940-4947.

162 G. Zhang, Z.-A. Lan and X. Wang, Chem. Sci., 2017, 8, 52615274.

163 A. Savateev, I. Ghosh, B. König and M. Antonietti, Angew. Chem., Int. Ed., 2018, 57, 15936-15947.
164 A. Savateev and M. Antonietti, ACS Catal., 2018, 8, 97909808.

165 Y. Guo, Y. Wang, S. Zhao, Z. Liu, H. Chang and X. Zhao, Chem. Eng. J., 2019, 369, 553-562.

166 B. Kurpil, Y. Markushyna and A. Savateev, ACS Catal., 2019, 9, 1531-1538.

167 B. Kurpil, K. Otte, A. Mishchenko, P. Lamagni, W. Lipiński, N. Lock, M. Antonietti and A. Savateev, Nat. Commun., 2019, $10,945$.

168 H. Liu, H. Li, J. Lu, S. Zeng, M. Wang, N. Luo, S. Xu and F. Wang, ACS Catal., 2018, 8, 4761-4771.

169 Y. Wang, X. Wang and M. Antonietti, Angew. Chem., Int. Ed., 2012, 51, 68-89.

170 C. Zhou, Z. Zeng, G. Zeng, D. Huang, R. Xiao, M. Cheng, C. Zhang, W. Xiong, C. Lai, Y. Yang, W. Wang, H. Yi and B. Li, J. Hazard. Mater., 2019, 120815.

171 M. Ghaemmaghami and R. Mohammadi, Sustainable Energy Fuels, 2019, 3, 2176-2204.

172 L. Chen and J. Song, Adv. Funct. Mater., 2017, 27, 1702695. 173 J. Sun, R. Malishev, A. Azoulay, J. Tzadikov, M. Volokh, R. Jelinek and M. Shalom, Small, 2018, 14, 1800516.

174 J. Xu and M. Shalom, ChemPhotoChem, 2019, 3, 170-179.

175 A.-J. Wang, H. Li, H. Huang, Z.-S. Qian and J.-J. Feng, J. Mater. Chem. C, 2016, 4, 8146-8160.

176 Y. Dong, Q. Wang, H. Wu, Y. Chen, C.-H. Lu, Y. Chi and H.-H. Yang, Small, 2016, 12, 5376-5393.

177 M. M. Xavier, P. R. Nair and S. Mathew, Analyst, 2019, 144, 1475-1491.

178 Y. Wang, R. Zhang, Z. Zhang, J. Cao and T. Ma, Adv. Mater. Interfaces, 2019, 1-32.

179 H. Wang, Y. Bu, G. Wu and X. Zou, Dalton Trans., 2019, 48, 11724-11731.

180 Y. Shiraishi, S. Shiota, Y. Kofuji, M. Hashimoto, K. Chishiro, H. Hirakawa, S. Tanaka, S. Ichikawa and T. Hirai, ACS Appl. Energy Mater., 2018, 1, 4169-4177.

181 X. Chen, X. Zhao, Z. Kong, W.-J. Ong and N. Li, J. Mater. Chem. A, 2018, 6, 21941-21948.

182 S. Cao, H. Chen, F. Jiang and X. Wang, Appl. Catal., B, 2018, 224, 222-229.

183 X. Chen, N. Li, Z. Kong, W.-J. Ong and X. Zhao, Mater. Horiz., 2018, 5, 9-27.

184 Y. Ren, D. Zeng and W.-J. Ong, Chin. J. Catal., 2019, 40, 289319.

185 G. Dong, W. Ho and C. Wang, J. Mater. Chem. A, 2015, 3, 23435-23441.

186 Z. Wang, X. Hu, Z. Liu, G. Zou, G. Wang and K. Zhang, ACS Catal., 2019, 9, 10260-10278.

187 Q. Cao, Q. Cui, Y. Yang, J. Xu, C. Han and L. Li, Chem.-Eur. J., 2018, 24, 2286-2291.

188 C. Han, P. Meng, E. R. Waclawik, C. Zhang, X.-H. Li, H. Yang, M. Antonietti and J. Xu, Angew. Chem., Int. Ed., 2018, 57, 14857-14861.

189 C. Han, Q. Cui, P. Meng, E. R. Waclawik, H. Yang and J. Xu, Langmuir, 2018, 34, 10135-10143.

190 G. Moon, M. Fujitsuka, S. Kim, T. Majima, X. Wang and W. Choi, ACS Catal., 2017, 7, 2886-2895. 
191 K. Villa, C. L. Manzanares Palenzuela, Z. Sofer, S. Matějková and M. Pumera, ACS Nano, 2018, 12, 12482-12491.

192 J. L. Segura, M. J. Mancheño and F. Zamora, Chem. Soc. Rev., 2016, 45, 5635-5671.

193 J. Zhang, X. Chen, K. Takanabe, K. Maeda, K. Domen, J. D. Epping, X. Fu, M. Antonietti and X. Wang, Angew. Chem., Int. Ed., 2010, 49, 441-444.

194 G. Li, Q. Wang, Y. Wu, Y. Li and L. Guo, Spectrochim. Acta, Part A, 2019, 215, 307-312.

195 L. Li, Q. Meng, H. Lv, L. Shui, Y. Zhang, Z. Zhang, Z. Chen, M. Yuan, R. Nötzel, X. Wang, J.-M. Liu and G. Zhou, Appl. Surf. Sci., 2018, 428, 739-747.

196 L. Chen, Z. Song, X. Liu, L. Guo, M. Li and F. Fu, Analyst, 2018, 143, 1609-1614.

197 Z. Jinshui, Z. Guigang, C. Xiufang, L. Sen, M. Lennart, D. Grzegorz, L. Grzegorz, A. Markus, B. Siegfried and W. Xinchen, Angew. Chem., 2012, 124, 3237-3241.

198 J. Zhang, M. Zhang, S. Lin, X. Fu and X. Wang, J. Catal., 2014, 310, 24-30.

199 J. Xu, Y. Wang and Y. Zhu, Langmuir, 2013, 29, 1056610572.

200 Y. Huang, D. Li, Z. Fang, R. Chen, B. Luo and W. Shi, Appl. Catal., B, 2019, 254, 128-134.

201 Z. Chen, P. Sun, B. Fan, Q. Liu, Z. Zhang and X. Fang, Appl. Catal., B, 2015, 170-171, 10-16.

202 J. Zhou, Y. Yang and C. Zhang, Chem. Commun., 2013, 49, 8605-8607.

203 Z. Song, Z. Li, L. Lin, Y. Zhang, T. Lin, L. Chen, Z. Cai, S. Lin, L. Guo, F. Fu and X. Wang, Nanoscale, 2017, 9, 1773717742.

204 X. L. Wang and H. G. Yang, Appl. Catal., B, 2017, 205, 624630.

205 G. Zhang and X. Wang, J. Catal., 2013, 307, 246-253.

206 K. Li and W.-D. Zhang, Small, 2018, 14, 1703599.

207 A. Hayat, F. Raziq, M. Khan, J. Khan, S. K. B. Mane, A. Ahmad, M. U. Rahman and W. U. Khan, J. Colloid Interface Sci., 2019, 554, 627-639.

208 K. Li, M. Sun and W.-D. Zhang, Carbon, 2018, 134, 134-144.

209 J. Qin, S. Wang, H. Ren, Y. Hou and X. Wang, Appl. Catal., B, 2015, 179, 1-8.

210 W. Yan, Y. Yu, H. Zou, X. Wang, P. Li, W. Gao, J. Wang, S. Wu and K. Ding, Sol. RRL, 2018, 2, 1800058.

211 G. Zhang, A. Savateev, Y. Zhao, L. Li and M. Antonietti, J. Mater. Chem. A, 2017, 5, 12723-12728.

212 H. Tang, R. Wang, C. Zhao, Z. Chen, X. Yang, D. Bukhvalov, Z. Lin and Q. Liu, Chem. Eng. J., 2019, 374, 1064-1075.

213 Y. Li, J. Zhang, Q. Wang, Y. Jin, D. Huang, Q. Cui and G. Zou, J. Phys. Chem. B, 2010, 114, 9429-9434.

214 L. Luo, M. Zhang, P. Wang, Y. Wang and F. Wang, New J. Chem., 2018, 42, 1087-1091.

215 W. Ho, Z. Zhang, W. Lin, S. Huang, X. Zhang, X. Wang and Y. Huang, ACS Appl. Mater. Interfaces, 2015, 7, 5497-5505.

216 M. Ismael, Y. Wu, D. H. Taffa, P. Bottke and M. Wark, New J. Chem., 2019, 43, 6909-6920.

217 G. Li, J. Shi, G. Zhang, Y. Fang, M. Anpo and X. Wang, Res. Chem. Intermed., 2017, 43, 5137-5152.
218 D. Vidyasagar, N. Manwar, A. Gupta, S. G. Ghugal, S. S. Umare and R. Boukherroub, Catal. Sci. Technol., 2019, 9, 822-832.

219 L. Zhou, H. Zhang, H. Sun, S. Liu, M. O. Tade, S. Wang and W. Jin, Catal. Sci. Technol., 2016, 6, 7002-7023.

220 L. Jiang, X. Yuan, Y. Pan, J. Liang, G. Zeng, Z. Wu and H. Wang, Appl. Catal., B, 2017, 217, 388-406.

221 B. X. Wang, X. Chen, A. Thomas, X. Fu and M. Antonietti, Adv. Mater., 2009, 1609-1612.

222 Z. Ding, X. Chen, M. Antonietti and X. Wang, ChemSusChem, 2011, 4, 274-281.

223 Z. Shouwei, L. Jiaxing, Z. Meiyi, L. Jie, X. Jinzhang and W. Xiangke, Chem.-Eur. J., 2014, 20, 9805-9812.

224 L.-F. Gao, T. Wen, J.-Y. Xu, X.-P. Zhai, M. Zhao, G.-W. Hu, P. Chen, Q. Wang and H.-L. Zhang, ACS Appl. Mater. Interfaces, 2016, 8, 617-624.

225 S. Hu, X. Chen, Q. Li, F. Li, Z. Fan, H. Wang, Y. Wang, B. Zheng and G. Wu, Appl. Catal., B, 2017, 201, 58-69.

226 Y. Yang, M. Guo, G. Zhang and W. Li, Carbon, 2017, 117, 120-125.

227 W. Wu, Z. Ruan, J. Li, Y. Li, Y. Jiang, X. Xu, D. Li, Y. Yuan and K. Lin, Nano-Micro Lett., 2019, 11, 10.

228 D. Das, D. Banerjee, M. Mondal, A. Shett, B. Das, N. S. Das, U. K. Ghorai and K. K. Chattopadhyay, Mater. Res. Bull., 2018, 101, 291-304.

229 G. Zhang, C. Huang and X. Wang, Small, 2015, 11, 12151221.

230 J. Mu, J. Li, X. Zhao, E.-C. Yang and X.-J. Zhao, RSC Adv., 2016, 6, 35568-35576.

231 P.-W. Chen, K. Li, Y.-X. Yu and W.-D. Zhang, Appl. Surf. Sci., 2017, 392, 608-615.

232 Y. Fu, W. Zhan, Y. Guo, Y. Guo, Y. Wang and G. Lu, Green Energy Environ., 2017, 2, 142-150.

233 W. Gong, J. Zou, S. Zhang, X. Zhou and J. Jiang, Electroanalysis, 2016, 28, 227-234.

234 X. Zou, R. Silva, A. Goswami and T. Asefa, Appl. Surf. Sci., 2015, 357, 221-228.

235 J.-C. Wang, C.-X. Cui, Y. Li, L. Liu, Y.-P. Zhang and W. Shi, J. Hazard. Mater., 2017, 339, 43-53.

236 Z. Li, C. Kong and G. Lu, J. Phys. Chem. C, 2015, 120, 56-63. 237 J.-C. Wang, C.-X. Cui, Q.-Q. Kong, C.-Y. Ren, Z. Li, L. Qu, Y. Zhang and K. Jiang, ACS Sustainable Chem. Eng., 2018, 6, 8754-8761.

238 G. Ding, W. Wang, T. Jiang, B. Han, H. Fan and G. Yang, ChemCatChem, 2013, 5, 192-200.

239 Y. Dai, Y. Gu and Y. Bu, Appl. Surf. Sci., 2020, 500, 144036. 240 K. Eid, Y. Ahmad, A. Mohamed, A. Elsafy and S. AlQaradawi, Catalysts, 2018, 8, 411.

241 K. Eid, M. H. Sliem, K. Jlassi, A. S. Eldesoky, G. G. Abdo, S. Y. Al-Qaradawi, M. A. Sharaf, A. M. Abdullah and A. A. Elzatahry, Inorg. Chem. Commun., 2019, 107460.

242 K. Eid, M. H. Sliem, A. S. Eldesoky, H. Al-Kandari and A. M. Abdullah, Int. J. Hydrogen Energy, 2019, 44, 1794317953.

243 K. Eid, M. H. Sliem, H. Al-Kandari, M. A. Sharaf and A. M. Abdullah, Langmuir, 2019, 35, 3421-3431. 
244 K. Eid, M. H. Sliem and A. M. Abdullah, Nanoscale, 2019, 11, 11755-11764.

245 C. Hun Choi, L. Lin, S. Gim, S. Lee, H. Kim, X. Wang and W. Choi, ACS Catal., 2018, 8, 4241-4256.

246 Z. Chen, S. Pronkin, T.-P. Fellinger, K. Kailasam, G. Vilé, D. Albani, F. Krumeich, R. Leary, J. Barnard, J. M. Thomas, J. Pérez-Ramírez, M. Antonietti and D. Dontsova, ACS Nano, 2016, 10, 3166-3175.

247 D. Xu, X. Li, J. Liu and L. Huang, J. Rare Earths, 2013, 31, 1085-1091.

248 Y. Wang, Y. Li, X. Bai, Q. Cai, C. Liu, Y. Zuo, S. Kang and L. Cui, Catal. Commun., 2016, 84, 179-182.

249 R. Jin, S. Hu, J. Gui and D. Liu, Bull. Korean Chem. Soc., 2015, 36, 17-23.

250 J. Xu, T. J. K. Brenner, Z. Chen, D. Neher, M. Antonietti and M. Shalom, ACS Appl. Mater. Interfaces, 2014, 6, 16481-16486.

251 D. Salinas, P. Araya and S. Guerrero, Appl. Catal., B, 2012, 117-118, 260-267.

252 H. Chu, L. Yang, Q. Zhang and Y. Wang, J. Catal., 2006, 241, 225-228.

253 G. Pekridis, N. Kaklidis, M. Konsolakis, C. Athanasiou, I. V. Yentekakis and G. E. Marnellos, Solid State Ionics, 2011, 192, 653-658.

254 Y. Chen, J. He, H. Tian, D. Wang and Q. Yang, J. Colloid Interface Sci., 2014, 428, 1-7.

255 J. Jiang, G. Lu, C. Miao, X. Wu, W. Wu and Q. Sun, Microporous Mesoporous Mater., 2013, 167, 213-220.

256 Y. Markushyna, C. A. Smith and A. Savateev, Eur. J. Org. Chem., 2020, 2020, 1294-1309.

257 J. Zhang, S. Hu and Y. Wang, RSC Adv., 2014, 4, 6291262919.

258 S. Hu, F. Li, Z. Fan, F. Wang, Y. Zhao and Z. Lv, Dalton Trans., 2015, 44, 1084-1092.

259 N. Hou, W.-M. Sun, F.-Y. Du and H.-S. Wu, Optik, 2019, 183, 455-462.

260 Y. Zhang, S. Zong, C. Cheng, J. Shi, X. Guan, Y. Lu and L. Guo, Int. J. Hydrogen Energy, 2018, 43, 13953-13961.

261 Y. Shang, Y. Ma, X. Chen, X. Xiong and J. Pan, Mol. Catal., 2017, 433, 128-135.

262 M. Zhang, X. Bai, D. Liu, J. Wang and Y. Zhu, Appl. Catal., B, 2015, 164, 77-81.

263 H. Yu, R. Shi, Y. Zhao, T. Bian, Y. Zhao, C. Zhou, G. I. N. Waterhouse, L.-Z. Wu, C.-H. Tung and T. Zhang, Adv. Mater., 2017, 29, 1605148.

264 L. Zhang, N. Ding, M. Hashimoto, K. Iwasaki, N. Chikamori, K. Nakata, Y. Xu, J. Shi, H. Wu, Y. Luo, D. Li, A. Fujishima and Q. Meng, Nano Res., 2018, 11, 2295-2309.

265 N. Zhou, P. Qiu, H. Chen and F. Jiang, J. Taiwan Inst. Chem. Eng., 2018, 83, 99-106.

266 H. Tan, X. Gu, P. Kong, Z. Lian, B. Li and Z. Zheng, Appl. Catal., B, 2019, 242, 67-75.

267 H. Tan, P. Kong, M. Liu, X. Gu and Z. Zheng, Chem. Commun., 2019, 55, 12503-12506.

268 W. Wang, H. Zhang, S. Zhang, Y. Liu, G. Wang, C. Sun and H. Zhao, Angew. Chem., Int. Ed., 2019, 58, 16644-16650.
269 L. Ruan, G. Xu, L. Gu, C. Li, Y. Zhu and Y. Lu, Mater. Res. Bull., 2015, 66, 156-162.

270 Q. Deng and Q. Li, J. Mater. Sci., 2018, 53, 506-515.

271 S. C. Yan, Z. S. Li and Z. G. Zou, Langmuir, 2010, 26, 38943901.

272 Y. Wang, Y. Di, M. Antonietti, H. Li, X. Chen and X. Wang, Chem. Mater., 2010, 22, 5119-5121.

273 Y. Wang, J. Zhang, X. Wang, M. Antonietti and H. Li, Angew. Chem., Int. Ed., 2010, 49, 3356-3359.

274 M. Jalaly, F. José Gotor, M. Semnan and M. Jesús Sayagués, Sci. Rep., 2017, 7, 3453.

275 S. Thaweesak, S. Wang, M. Lyu, M. Xiao, P. Peerakiatkhajohn and L. Wang, Dalton Trans., 2017, 46, 10714-10720.

276 Y. Wang, H. Li, J. Yao, X. Wang and M. Antonietti, Chem. Sci., 2011, 2, 446-450.

277 Y. Luo, J. Wang, S. Yu, Y. Cao, K. Ma, Y. Pu, W. Zou, C. Tang, F. Gao and L. Dong, J. Mater. Res., 2018, 33, 1268-1278.

278 Z. Lin and X. Wang, Angew. Chem., Int. Ed., 2013, 52, 17351738.

279 J. Zou, Y. Yu, W. Yan, J. Meng, S. Zhang and J. Wang, J. Mater. Sci., 2019, 54, 6867-6881.

280 C. Lu, R. Chen, X. Wu, M. Fan, Y. Liu, Z. Le, S. Jiang and S. Song, Appl. Surf. Sci., 2016, 360, 1016-1022.

281 J. Zhu, T. Diao, W. Wang, X. Xu, X. Sun, S. A. C. Carabineiro and Z. Zhao, Appl. Catal., B, 2017, 219, 92-100.

282 J. Hong, X. Xia, Y. Wang and R. Xu, J. Mater. Chem., 2012, 22, 15006-15012.

283 J. Chen, Z. Hong, Y. Chen, B. Lin and B. Gao, Mater. Lett., 2015, 145, 129-132.

284 K.-Y. A. Lin, Z.-Y. Zhang and T. Wi-Afedzi, J. Water Process Eng., 2018, 24, 83-89.

285 C. Rajkumar, P. Veerakumar, S.-M. Chen, B. Thirumalraj and K.-C. Lin, ACS Sustainable Chem. Eng., 2018, 6, 16021-16031.

286 K.-Y. A. Lin and Z.-Y. Zhang, Chem. Eng. J., 2017, 313, 13201327.

287 J. Jin, H. Wu, S. Wang, Y. Ding and S. Yin, Int. J. Hydrogen Energy, 2017, 42, 20579-20588.

288 K. Wang, Q. Li, B. Liu, B. Cheng, W. Ho and J. Yu, Appl. Catal., B, 2015, 176-177, 44-52.

289 X. Dai, Z. Han, H. Fan and S. Ai, J. Nanopart. Res., 2018, 20, 315.

290 R. Zhu, Y. Zhang, X. Fang, X. Cui, J. Wang, C. Yue, W. Fang, H. Zhao and Z. Li, J. Mater. Chem. B, 2019, 7, 2320-2329.

291 F. Qiao, J. Wang, S. Ai and L. Li, Sens. Actuators, B, 2015, 216, 418-427.

292 A. Kumar, R. K. Yadav, N.-J. Park and J.-O. Baeg, ACS Appl. Nano Mater., 2017, 1, 47-54.

293 X. Ma, Y. Lv, J. Xu, Y. Liu, R. Zhang and Y. Zhu, J. Phys. Chem. C, 2012, 116, 23485-23493.

294 Y. Zhang, T. Mori, J. Ye and M. Antonietti, J. Am. Chem. Soc., 2010, 132, 6294-6295.

295 L. Zhang, X. Chen, J. Guan, Y. Jiang, T. Hou and X. Mu, Mater. Res. Bull., 2013, 48, 3485-3491.

296 S. Hu, L. Ma, J. You, F. Li, Z. Fan, F. Wang, D. Liu and J. Gui, RSC Adv., 2014, 4, 21657-21663. 
297 S. Hu, L. Ma, J. You, F. Li, Z. Fan, G. Lu, D. Liu and J. Gui, Appl. Surf. Sci., 2014, 311, 164-171.

298 B. Chai, J. Yan, C. Wang, Z. Ren and Y. Zhu, Appl. Surf. Sci., 2017, 391, 376-383.

299 J. Ran, T. Y. Ma, G. Gao, X.-W. Du and S. Z. Qiao, Energy Environ. Sci., 2015, 8, 3708-3717.

300 Y.-P. Zhu, T.-Z. Ren and Z.-Y. Yuan, ACS Appl. Mater. Interfaces, 2015, 7, 16850-16856.

301 Y. Zhou, L. Zhang, J. Liu, X. Fan, B. Wang, M. Wang, W. Ren, J. Wang, M. Li and J. Shi, J. Mater. Chem. A, 2015, 3, 3862-3867.

302 W. Zhang, J. Zhang, F. Dong and Y. Zhang, RSC Adv., 2016, 6, 88085-88089.

303 W. Zhang, J. Barrio, C. Gervais, A. Kocjan, A. Yu, X. Wang and M. Shalom, Angew. Chem., Int. Ed., 2018, 57, 9764-9769.

304 Z. Liang, Y. Xia, G. Ba, H. Li, Q. Deng and W. Hou, Catal. Sci. Technol., 2019, 9, 6627-6637.

305 J. Cui, S. Liang, X. Wang and J. Zhang, Mater. Chem. Phys., 2015, 161, 194-200.

306 P. Qiu, C. Xu, H. Chen, F. Jiang, X. Wang, R. Lu and X. Zhang, Appl. Catal., B, 2017, 206, 319-327.

307 Y.-C. Lu, J. Chen, A.-J. Wang, N. Bao, J.-J. Feng, W. Wang and L. Shao, J. Mater. Chem. C, 2015, 3, 73-78.

308 L. Jiang, X. Yuan, G. Zeng, X. Chen, Z. Wu, J. Liang, J. Zhang, H. Wang and H. Wang, ACS Sustainable Chem. Eng., 2017, 5, 5831-5841.

309 X. Han, A. Yuan, C. Yao, F. Xi, J. Liu and X. Dong, J. Mater. Sci., 2019, 54, 1593-1605.

310 Q. Liu, J. Shen, X. Yu, X. Yang, W. Liu, J. Yang, H. Tang, H. Xu, H. Li, Y. Li and J. Xu, Appl. Catal., B, 2019, 248, 84-94.

311 B. Zhu, J. Zhang, C. Jiang, B. Cheng and J. Yu, Appl. Catal., $B, 2017,207,27-34$.

312 Z. Guigang, Z. Mingwen, Y. Xinxin, Q. Xiaoqing, L. Sen and W. Xinchen, Adv. Mater., 2013, 26, 805-809.

313 Y. Guo, T. Chen, Q. Liu, Z. Zhang and X. Fang, J. Phys. Chem. C, 2016, 120, 25328-25337.

314 Z. A. Lan, G. Zhang and X. Wang, Appl. Catal., B, 2016, 192, 116-125.

315 Y. Zhu, L. Gong, D. Zhang, X. Wang, J. Zhang, L. Zhang, L. Dai and Z. Xia, Nano Energy, 2019, 63, 103819.

316 G. Liu, P. Niu, C. Sun, S. C. Smith, Z. Chen, G. Qing (Max) Lu and H.-M. Cheng, J. Am. Chem. Soc., 2010, 132, 1164211648.

317 H. Ou, C. Tang, Y. Zhang, A. M. Asiri, M.-M. Titirici and X. Wang, J. Catal., 2019, 375, 104-112.

318 J. Cao, J. Zhang, X. Dong, H. Fu, X. Zhang, X. Lv, Y. Li and G. Jiang, Appl. Catal., B, 2019, 249, 266-274.

319 B. Wang, H. Cai, D. Zhao, M. Song, P. Guo, S. Shen, D. Li and S. Yang, Appl. Catal., B, 2019, 244, 486-493.

320 A. Tripathi and S. Narayanan, Appl. Surf. Sci., 2019, 479, 111.

321 W. Xu, X. An, Q. Zhang, Z. Li, Q. Zhang, Z. Yao, X. Wang, S. Wang, J. Zheng, J. Zhang, W. Wu and M. Wu, ACS Sustainable Chem. Eng., 2019, 7, 12351-12357.
322 E. Ju, K. Dong, Z. Chen, Z. Liu, C. Liu, Y. Huang, Z. Wang, F. Pu, J. Ren and X. Qu, Angew. Chem., Int. Ed., 2016, 55, 11467-11471.

323 M. Vázquez-González, W.-C. Liao, R. Cazelles, S. Wang, X. Yu, V. Gutkin and I. Willner, ACS Nano, 2017, 11, 3247-3253.

324 P. Sharma and Y. Sasson, Green Chem., 2017, 19, 844-852.

325 Y. Wang, X. Zhou, W. Xu, Y. Sun, T. Wang, Y. Zhang, J. Dong, W. Hou, N. Wu, L. Wu, B. Zhou, Y. Wu, Y. Du and W. Zhong, Appl. Catal., A, 2019, 582, 117118.

326 H. Zhang, Y. Huang, X. Lin, F. Lu, Z. Zhang and Z. Hu, Sens. Actuators, B, 2018, 255, 2218-2222.

327 Q. Han, C. Hu, F. Zhao, Z. Zhang, N. Chen and L. Qu, J. Mater. Chem. A, 2015, 3, 4612-4619.

328 Z. Yang, Y. Zhang and Z. Schnepp, J. Mater. Chem. A, 2015, 3, 14081-14092.

329 A. Thomas, F. Goettmann and M. Antonietti, Chem. Mater., 2008, 20, 738-755.

330 M. Groenewolt and M. Antonietti, Adv. Mater., 2005, 17, 1789-1792.

331 F. Goettmann, A. Fischer, M. Antonietti and A. Thomas, Angew. Chem., Int. Ed., 2006, 45, 4467-4471.

332 M. Kruk, M. Jaroniec, C. Hyun Ko and R. Ryoo, Chem. Mater., 2000, 12, 1961-1968.

333 A. Vinu, Adv. Funct. Mater., 2008, 18, 816-827.

334 S.-W. Bian, Z. Ma and W.-G. Song, J. Phys. Chem. C, 2009, 113, 8668-8672.

335 X. Jin, V. V. Balasubramanian, S. T. Selvan, D. P. Sawant, M. A. Chari, G. Q. Lu and A. Vinu, Angew. Chem., Int. Ed., 2009, 48, 7884-7887.

336 X.-H. Li, J. Zhang, X. Chen, A. Fischer, A. Thomas, M. Antonietti and X. Wang, Chem. Mater., 2011, 23, 43444348.

337 J. Liu, J. Huang, H. Zhou and M. Antonietti, ACS Appl. Mater. Interfaces, 2014, 6, 8434-8440.

338 J. Sun, J. Zhang, M. Zhang, M. Antonietti, X. Fu and X. Wang, Nat. Commun., 2012, 3, 1139.

339 Y. Si, Z. Sun, L. Huang, M. Chen and L. Wu, J. Mater. Chem. A, 2019, 7, 8952-8959.

340 M. Shalom, S. Inal, D. Neher and M. Antonietti, Catal. Today, 2014, 225, 185-190.

341 T. Fu, M. Wang, W. Cai, Y. Cui, F. Gao, L. Peng, W. Chen and W. Ding, ACS Catal., 2014, 4, 2536-2543.

342 D. Chen, J. Yang and H. Ding, Appl. Surf. Sci., 2017, 391, 384-391.

343 L. Shi, L. Liang, F. Wang, M. Liu, K. Chen, K. Sun, N. Zhang and J. Sun, ACS Sustainable Chem. Eng., 2015, 3, 3412-3419.

344 J. Zhang, M. Zhang, C. Yang and X. Wang, Adv. Mater., 2014, 26, 4121-4126.

345 M. Hu, J. Reboul, S. Furukawa, L. Radhakrishnan, Y. Zhang, P. Srinivasu, H. Iwai, H. Wang, Y. Nemoto, N. Suzuki, S. Kitagawa and Y. Yamauchi, Chem. Commun., 2011, 47, 8124-8126.

346 J. Liu, J. Yan, H. Ji, Y. Xu, L. Huang, Y. Li, Y. Song, Q. Zhang, H. Xu and H. Li, Mater. Sci. Semicond. Process., 2016, 46, 5968. 
347 H.-M. Zhao, C.-M. Di, L. Wang, Y. Chun and Q.-H. Xu, Microporous Mesoporous Mater., 2015, 208, 98-104.

348 J. Zhang, F. Guo and X. Wang, Adv. Funct. Mater., 2013, 23, 3008-3014.

349 Y. Ling, G. Liao, W. Feng, Y. Liu and L. Li, J. Photochem. Photobiol., A, 2017, 349, 108-114.

350 M. Jourshabani, Z. Shariatinia and A. Badiei, Langmuir, 2017, 33, 7062-7078.

351 X. Chen, J. Zhang, X. Fu, M. Antonietti and X. Wang, J. Am. Chem. Soc., 2009, 131, 11658-11659.

352 S. Hu, X. Qu, P. Li, F. Wang, Q. Li, L. Song, Y. Zhao and X. Kang, Chem. Eng. J., 2018, 334, 410-418.

353 S. Le, T. Jiang, Q. Zhao, X. Liu, Y. Li, B. Fang and M. Gong, RSC Adv., 2016, 6, 38811-38819.

354 Y. Guo, Q. Liu, Z. Li, Z. Zhang and X. Fang, Appl. Catal., B, 2018, 221, 362-370.

355 K. Leng, W. Mai, X. Zhang, R. Liu, X. Lin, J. Huang, H. Lou, Y. Xie, R. Fu and D. Wu, Chem. Commun., 2018, 54, 71597162.

356 Y. Chen, J. Zhang, M. Zhang and X. Wang, Chem. Sci., 2013, 4, 3244 .

357 W. Yong, W. Xinchen, A. Markus and Z. Yuanjian, ChemSusChem, 2010, 3, 435-439.

358 H. Yan, Chem. Commun., 2012, 48, 3430.

359 F. He, G. Chen, Y. Zhou, Y. Yu, Y. Zheng and S. Hao, Chem. Commun., 2015, 51, 16244-16246.

360 Y. Zhang, Z. Schnepp, J. Cao, S. Ouyang, Y. Li, J. Ye and S. Liu, Sci. Rep., 2013, 3, 2163.

361 M. A. Mohamed, M. F. M. Zain, L. J. Minggu, M. B. Kassim, J. Jaafar, N. A. Saidina Amin, Z. A. Mohd Hir and M. S. Rosmi, Int. J. Hydrogen Energy, 2019, 44, 13098-13105.

362 X. Zhao, Y. Zhang, X. Zhao, X. Wang, Y. Zhao, H.-Q. Tan, H. Zhu, W. Ho, H. Sun and Y.-G. Li, ACS Appl. Mater. Interfaces, 2019, 11, 27934-27943.

363 C. Merschjann, S. Tschierlei, T. Tyborski, K. Kailasam, S. Orthmann, D. Hollmann, T. Schedel-Niedrig, A. Thomas and S. Lochbrunner, Adv. Mater., 2015, 27, 7993-7999.

364 C. Merschjann, T. Tyborski, S. Orthmann, F. Yang, K. Schwarzburg, M. Lublow, M.-C. Lux-Steiner and T. Schedel-Niedrig, Phys. Rev. B: Condens. Matter Mater. Phys., 2013, 87, 205204.

365 M. Wu, J.-M. Yan, X. Tang, M. Zhao and Q. Jiang, ChemSusChem, 2014, 7, 2654-2658.

366 L. Shi, L. Yao and W. Si, J. Mater. Sci.: Mater. Electron., 2019, 30, 1714-1723.

367 A. Savateev, Z. P. Chen and D. Dontsova, RSC Adv., 2016, 6, 2910-2913.

368 C. Liu, Y. Zhang, F. Dong, A. H. Reshak, L. Ye, N. Pinna, C. Zeng, T. Zhang and H. Huang, Appl. Catal., B, 2017, 203, 465-474.

369 Z. Jun-Jun, G. Jie-Min, W. Hong-Hui, W. Xiao, L. Xin-Hao and C. Jie-Sheng, ChemCatChem, 2016, 8, 3441-3445.

370 X. Qian, X. Meng, J. Sun, L. Jiang, Y. Wang, J. Zhang, X. Hu, M. Shalom and J. Zhu, ACS Appl. Mater. Interfaces, 2019, 11, 27226-27232.
371 L. Lin, Z. Yu and X. Wang, Angew. Chem., Int. Ed., 2019, 58, 6164-6175.

372 A. Savateev and M. Antonietti, ChemCatChem, 2019, 11, 6166-6176.

373 X. Liu, N. Fechler and M. Antonietti, Chem. Soc. Rev., 2013, 42, 8237-8265.

374 B. P. Jelle and S. E. Kalnæs, Cost-Effective Energy Effic. Build. Retrofit., 2017, pp. 57-118.

375 P. Kuhn, M. Antonietti and A. Thomas, Angew. Chem., Int. Ed., 2008, 47, 3450-3453.

376 M. J. Bojdys, J.-O. Müller, M. Antonietti and A. Thomas, Chem.-Eur. J., 2008, 14, 8177-8182.

377 E. Wirnhier, M. Döblinger, D. Gunzelmann, J. Senker, B. V Lotsch and W. Schnick, Chem.-Eur. J., 2011, 17, 3213-3221.

378 G. Algara-Siller, N. Severin, S. Y. Chong, T. Björkman, R. G. Palgrave, A. Laybourn, M. Antonietti, Y. Z. Khimyak, A. V Krasheninnikov, J. P. Rabe, U. Kaiser, A. I. Cooper, A. Thomas and M. J. Bojdys, Angew. Chem., Int. Ed., 2014, 53, 7450-7455.

379 S. Y. Chong, J. T. A. Jones, Y. Z. Khimyak, A. I. Cooper, A. Thomas, M. Antonietti and M. J. Bojdys, J. Mater. Chem. A, 2013, 1, 1102-1107.

380 L. Lin, C. Wang, W. Ren, H. Ou, Y. Zhang and X. Wang, Chem. Sci., 2017, 8, 5506-5511.

381 Y. Ham, K. Maeda, D. Cha, K. Takanabe and K. Domen, Chem.-Asian J., 2013, 8, 218-224.

382 A. Belen Jorge, D. James Martin, M. T. S. Dhanoa, A. S. Rahman, N. Makwana, J. Tang, A. Sella, F. Corà, S. Firth, J. A. Darr and P. F. McMillan, J. Phys. Chem. C, 2013, 117, 7178-7185.

383 K. Schwinghammer, M. B. Mesch, V. Duppel, C. Ziegler, J. Senker and B. V Lotsch, J. Am. Chem. Soc., 2014, 136, 1730-1733.

384 M. K. Bhunia, K. Yamauchi and K. Takanabe, Angew. Chem., Int. Ed., 2014, 53, 11001-11005.

385 E. J. McDermott, E. Wirnhier, W. Schnick, K. Singh Virdi, C. Scheu, Y. Kauffmann, W. D. Kaplan, E. Z. Kurmaev and A. Moewes, J. Phys. Chem. C, 2013, 117, 8806-8812.

386 J. Zhao, L. Ma, H. Wang, Y. Zhao, J. Zhang and S. Hu, Appl. Surf. Sci., 2015, 332, 625-630.

387 C. Fettkenhauer, J. Weber, M. Antonietti and D. Dontsova, RSC Adv., 2014, 4, 40803-40811.

388 X. Yan, Z. Jia, H. Che, S. Chen, P. Hu, J. Wang and L. Wang, Appl. Catal., B, 2018, 234, 19-25.

389 C. Fettkenhauer, G. Clavel, K. Kailasam, M. Antonietti and D. Dontsova, Green Chem., 2015, 17, 3350-3361.

390 Y. Wang, Y. Li, W. Ju, J. Wang, H. Yao, L. Zhang, J. Wang and Z. Li, Carbon, 2016, 102, 477-486.

391 L. Heymann, B. Schiller, H. Noei, A. Stierle and C. Klinke, ACS Omega, 2018, 3, 3892-3900.

392 A. Savateev, S. Pronkin, M. G. Willinger, M. Antonietti and D. Dontsova, Chem.-Asian J., 2017, 12, 1517-1522.

393 B. Kurpil, A. Savateev, V. Papaefthimiou, S. Zafeiratos, T. Heil, S. Özenler, D. Dontsova and M. Antonietti, Appl. Catal., B, 2017, 217, 622-628. 
394 A. Savateev, S. Pronkin, J. D. Epping, M. G. Willinger, C. Wolff, D. Neher, M. Antonietti and D. Dontsova, ChemCatChem, 2017, 9, 167-174.

395 G. Zhang, G. Li, T. Heil, S. Zafeiratos, F. Lai, A. Savateev, M. Antonietti and X. Wang, Angew. Chem., Int. Ed., 2019, 58, 3433-3437.

396 Z. Chen, A. Savateev, S. Pronkin, V. Papaefthimiou, C. Wolff, M. G. Willinger, E. Willinger, D. Neher, M. Antonietti and D. Dontsova, Adv. Mater., 2017, 29, 1700555.

397 A. Savateev, D. Dontsova, B. Kurpil and M. Antonietti, J. Catal., 2017, 350, 203-211.

398 Y. Markushyna, C. Teutloff, B. Kurpil, D. Cruz, I. Lauermann, Y. Zhao, M. Antonietti and A. Savateev, Appl. Catal., B, 2019, 248, 211-217.

399 I. Ghosh, J. Khamrai, A. Savateev, N. Shlapakov, M. Antonietti and B. König, Science, 2019, 365, 360.

400 A. Schwarzer, T. Saplinova and E. Kroke, Coord. Chem. Rev., 2013, 257, 2032-2062.

401 L. Lin, H. Ou, Y. Zhang and X. Wang, ACS Catal., 2016, 6, 3921-3931.

402 G. Zhang, G. Li, Z.-A. Lan, L. Lin, A. Savateev, T. Heil, S. Zafeiratos, X. Wang and M. Antonietti, Angew. Chem., Int. Ed., 2017, 56, 13445-13449.

403 L. Jiang, X. Yuan, G. Zeng, J. Liang, Z. Wu, H. Wang, J. Zhang, T. Xiong and H. Li, Environ. Sci.: Nano, 2018, 5, 2604-2617.

404 J. Zhang, M. Zhang, R.-Q. Sun and X. Wang, Angew. Chem., Int. Ed., 2012, 51, 10145-10149.

405 A. Jin, Y. Jia, C. Chen, X. Liu, J. Jiang, X. Chen and F. Zhang, J. Phys. Chem. C, 2017, 121, 21497-21509.

406 X. Li, S. A. Bartlett, J. M. Hook, I. Sergeyev, E. B. Clatworthy, A. F. Masters and T. Maschmeyer, J. Mater. Chem. A, 2019, 7, 18987-18995.

407 J. Lehn, Angew. Chem., Int. Ed., 1988, 27, 89-112.

408 G. M. Whitesides and B. Grzybowski, Science, 2002, 295, 2418-2421.

409 J. Barrio and M. Shalom, ChemCatChem, 2018, 10, 55735586.

410 J. Xu, G. Wu, Z. Wang and X. Zhang, Chem. Sci., 2012, 3, 3227. 411 L. Xie, Y. Ding, X. Wang and W. Xu, Phys. Chem. Chem. Phys., 2019, 21, 9357-9361.

412 H. Yan, Y. Chen and S. Xu, Int. J. Hydrogen Energy, 2012, 37, 125-133.

413 J. Shen, H. Yang, Q. Shen and Y. Feng, Eur. J. Inorg. Chem., 2015, 2611-2618.

414 N. D. Shcherban, S. M. Filonenko, M. L. Ovcharov, A. M. Mishura, M. A. Skoryk, A. Aho and D. Y. Murzin, ChemistrySelect, 2016, 1, 4987-4993.

415 G. Dong and L. Zhang, J. Mater. Chem., 2012, 22, 1160-1166. 416 X. Wu, C. Liu, X. Li, X. Zhang, C. Wang and Y. Liu, Mater. Sci. Semicond. Process., 2015, 32, 76-81.

417 X. Jiang, J. Li, J. Fang, L. Gao, W. Cai, X. Li, A. Xu and X. Ruan, J. Photochem. Photobiol., A, 2017, 336, 54-62.

418 M. Wu, Y. Gong, T. Nie, J. Zhang, R. Wang, H. Wang and B. He, J. Mater. Chem. A, 2019, 7, 5324-5332.
419 S. Sun, E. Fan, H. Xu, W. Cao, G. Shao, B. Fan, H. Wang and R. Zhang, Nanotechnology, 2019, 30, 315601.

420 J. Barrio and M. Shalom, Mater. Sci. Semicond. Process., 2018, 73, 78-82.

421 J. Barrio, L. Lin, X. Wang and M. Shalom, ACS Sustainable Chem. Eng., 2018, 6, 519-530.

422 Y. Hong, C. Li, D. Li, Z. Fang, B. Luo, X. Yan, H. Shen, B. Mao and W. Shi, Nanoscale, 2017, 9, 14103-14110.

423 L. Zhang, N. Ding, J. Wu, K. Iwasaki, L. Lin, Y. Yamaguchi, Y. Shibayama, J. Shi, H. Wu, Y. Luo, K. Nakata, D. Li, X. Wang, A. Fujishima and Q. Meng, Catal. Sci. Technol., 2018, 8, 3846-3852.

424 J. Barrio, A. Grafmüller, J. Tzadikov and M. Shalom, Appl. Catal., B, 2018, 237, 681-688.

425 B. Zhu, G. Xu, X. Li, Z. Wang, J. Lv, X. Shu, J. Huang, Z. Zheng and Y. Wu, J. Mater. Res., 2019, 1-12.

426 M. Tahir, C. Cao, F. K. Butt, S. Butt, F. Idrees, Z. Ali, I. Aslam, M. Tanveer, A. Mahmood and N. Mahmood, CrystEngComm, 2014, 16, 1825.

427 S. Wan, M. Ou, Y. Wang, Y. Zeng, Y. Xiong, F. Song, J. Ding, W. Cai, S. Zhang and Q. Zhong, Appl. Catal., B, 2019, 258, 118011.

428 M. Tahir, C. Cao, N. Mahmood, F. K. Butt, A. Mahmood, F. Idrees, S. Hussain, M. Tanveer, Z. Ali and I. Aslam, ACS Appl. Mater. Interfaces, 2014, 6, 1258-1265.

429 M. Tahir, N. Mahmood, J. Zhu, A. Mahmood, F. K. Butt, S. Rizwan, I. Aslam, M. Tanveer, F. Idrees, I. Shakir, C. Cao and Y. Hou, Sci. Rep., 2015, 5, 12389.

430 J. Huang, Y. Cao, H. Wang, H. Yu, F. Peng, H. Zou and Z. Liu, Chem. Eng. J., 2019, 373, 687-699.

431 L. Shi, F. Wang, L. Liang, K. Chen, M. Liu, R. Zhu and J. Sun, Catal. Commun., 2017, 89, 129-132.

432 G. Ge and Z. Zhao, Catal. Sci. Technol., 2019, 9, 266-270.

433 Q. Chen, H. Dou, S. Zheng, X. Rao and Y. Zhang, J. Photochem. Photobiol., A, 2019, 382, 111931.

434 X. Gao, J. Feng, D. Su, Y. Ma, G. Wang, H. Ma and J. Zhang, Nano Energy, 2019, 59, 598-609.

435 H. Li, H. Tian, X. Wang, M. Pi, S. Wei, H. Zhu, D. Zhang and S. Chen, ACS Appl. Energy Mater., 2019, 2, 4692-4699.

436 Q. Weng, X. Wang, C. Zhi, Y. Bando and D. Golberg, ACS Nano, 2013, 7, 1558-1565.

437 L. Chen, M. Zhou, Z. Luo, M. Wakeel, A. M. Asiri and X. Wang, Appl. Catal., B, 2019, 241, 246-255.

438 A. Roy, A. Choudhury and C. N. R. Rao, J. Mol. Struct., 2002, 613, 61-66.

439 Y.-S. Jun, E. Z. Lee, X. Wang, W. H. Hong, G. D. Stucky and A. Thomas, Adv. Funct. Mater., 2013, 23, 3661-3667.

440 Y.-S. Jun, J. Park, S. U. Lee, A. Thomas, W. H. Hong and G. D. Stucky, Angew. Chem., Int. Ed., 2013, 23, 3661-3667.

441 C. T. Seto and G. M. Whitesides, J. Am. Chem. Soc., 1990, 112, 6409-6411.

442 J. a. Zerkowski, C. T. Seto and G. M. Whitesides, J. Am. Chem. Soc., 1992, 114, 5473-5475.

443 B. Roy, P. Bairi and A. K. Nandi, RSC Adv., 2014, 4, 1708-1734. 444 C. Li, B. J. Cafferty, S. C. Karunakaran, G. B. Schuster and N. V. Hud, Phys. Chem. Chem. Phys., 2016, 18, 20091-20096. 
445 M. Shalom, S. Inal, C. Fettkenhauer, D. Neher and M. Antonietti, J. Am. Chem. Soc., 2013, 135, 7118-7121.

446 Z. Sun, W. Wang, Q. Chen, Y. Pu, H. He, W. Zhuang, J. He and L. Huang, J. Mater. Chem. A, 2020, 8, 3160-3167.

447 B. Shen, Z. Hong, Y. Chen, B. Lin and B. Gao, Mater. Lett., 2014, 118, 208-211.

448 J. Hu, J. Tian and C. Li, ACS Appl. Mater. Interfaces, 2017, 9, 11615-11625.

449 N.-N. Vu, C.-C. Nguyen, S. Kaliaguine and T.-O. Do, ChemSusChem, 2019, 12, 291-302.

450 M. Shalom, M. Guttentag, C. Fettkenhauer, S. Inal, D. Neher, A. Llobet and M. Antonietti, Chem. Mater., 2014, 26, 5812-5818.

451 Q. Zheng, D. P. Durkin, J. E. Elenewski, Y. Sun, N. A. Banek, L. Hua, H. Chen, M. J. Wagner, W. Zhang and D. Shuai, Environ. Sci. Technol., 2016, 50, 12938-12948.

452 C. Zhou, C. Lai, D. Huang, G. Zeng, C. Zhang, M. Cheng, L. Hu, J. Wan, W. Xiong, M. Wen, X. Wen and L. Qin, Appl. Catal., B, 2018, 220, 202-210.

453 Y. Ishida, L. Chabanne, M. Antonietti and M. Shalom, Langmuir, 2014, 30, 447-451.

454 D. Tang, Y. Chen, M. Yin, Q. Yang, Y. Zhou and L. Zhou, Mater. Sci. Semicond. Process., 2020, 105, 104735.

455 C. Zhao, Z. Chen, J. Xu, Q. Liu, H. Xu, H. Tang, G. Li, Y. Jiang, F. Qu, Z. Lin and X. Yang, Appl. Catal., B, 2019, 256, 117867.

456 N. Karjule, J. Barrio, J. Tzadikov and M. Shalom, Chem.-Eur. J., 2020, DOI: 10.1002/chem.201905875.

457 Y. Liao, S. Zhu, J. Ma, Z. Sun, C. Yin, C. Zhu, X. Lou and D. Zhang, ChemCatChem, 2014, 6, 3419-3425.

458 T. Jordan, N. Fechler, J. Xu, T. J. K. Brenner, M. Antonietti and M. Shalom, ChemCatChem, 2015, 7, 2826-2830.

459 S. Wan, Q. Zhong, M. Ou and S. Zhang, RSC Adv., 2016, 6, 101208-101215.

460 S. Wan, M. Ou, Q. Zhong, S. Zhang and W. Cai, Adv. Opt. Mater., 2017, 5, 1700536.

461 X. Song, D. Tang, Y. Chen, M. Yin, Q. Yang, Z. Chen and L. Zhou, ACS Omega, 2019, 4, 6114-6125.

462 Y. Chen, F. Ding, A. Khaing, D. Yang and Z. Jiang, Appl. Surf. Sci., 2019, 479, 757-764.

463 W. Che, W. Cheng, T. Yao, F. Tang, W. Liu, H. Su, Y. Huang, Q. Liu, J. Liu, F. Hu, Z. Pan, Z. Sun and S. Wei, J. Am. Chem. Soc., 2017, 139, 3021-3026.

464 S. Guo, Z. Deng, M. Li, B. Jiang, C. Tian, Q. Pan and H. Fu, Angew. Chem., Int. Ed., 2016, 55, 1830-1834.

465 Z. Wang, M. Chen, Y. Huang, X. Shi, Y. Zhang, T. Huang, J. Cao, W. Ho and S. C. Lee, Appl. Catal., B, 2018, 239, 352-361.

466 S. Wang, F. He, X. Zhao, J. Zhang, Z. Ao, H. Wu, Y. Yin, L. Shi, X. Xu, C. Zhao, S. Wang and H. Sun, Appl. Catal., B, 2019, 257, 117931.

467 L. Yin, S. Wang, C. Yang, S. Lyu and X. Wang, ChemSusChem, 2019, 12, 3320-3325.

468 J. Xu, T. J. K. Brenner, L. Chabanne, D. Neher, M. Antonietti and M. Shalom, J. Am. Chem. Soc., 2014, 136, 13486-13489.

469 Q. Cui, J. Xu, X. Wang, L. Li, M. Antonietti and M. Shalom, Angew. Chem., Int. Ed., 2016, 55, 3672-3676.
470 L.-L. Feng, Y. Zou, C. Li, S. Gao, L.-J. Zhou, Q. Sun, M. Fan, H. Wang, D. Wang, G.-D. Li and X. Zou, Int. J. Hydrogen Energy, 2014, 39, 15373-15379.

471 Q. Fan, J. Liu, Y. Yu, S. Zuo and B. Li, Appl. Surf. Sci., 2017, 391, 360-368.

472 Y. Zheng, Z. Yu, F. Lin, F. Guo, A. K. Alamry, A. L. Taib, M. A. Asiri and X. Wang, Molecules, 2017, 22, 572.

473 H. Wang, Y. Bian, J. Hu and L. Dai, Appl. Catal., B, 2018, 238, 592-598.

474 J.-Y. Song, H.-J. Kang, J. C. Won, Y. H. Kim, Y.-S. Jun and H. S. Jeong, Mater. Horiz., 2019, 6, 1726-1732.

475 X. Wang, C. Zhou, R. Shi, Q. Liu, G. I. N. Waterhouse, L. Wu, C. H. Tung and T. Zhang, Nano Res., 2019, 12, 2385-2389.

476 P. Zhang, X. Li, C. Shao and Y. Liu, J. Mater. Chem. A, 2015, 3, 3281-3284.

477 X. Han, L. Tian, H. Jiang, L. Kong, J. Lv, J. Shan, J. Wang and X. Fan, RSC Adv., 2017, 7, 14372-14381.

478 H. Li, F. Li, Z. Wang, Y. Jiao, Y. Liu, P. Wang, X. Zhang, X. Qin, Y. Dai and B. Huang, Appl. Catal., B, 2018, 229, 114-120.

479 Y. Qin, Y. Ding and H. Tang, J. Environ. Chem. Eng., 2016, 4, 4374-4384.

480 Z. Chen, T.-T. Fan, X. Yu, Q.-L. Wu, Q.-H. Zhu, L.-Z. Zhang, J.-H. Li, W.-P. Fang and X.-D. Yi, J. Mater. Chem. A, 2018, 6, 15310-15319.

481 N. Tian, K. Xiao, Y. Zhang, X. Lu, L. Ye, P. Gao, T. Ma and H. Huang, Appl. Catal., B, 2019, 253, 196-205.

482 L. Li, Y. Zhao, M. Antonietti and M. Shalom, Small, 2016, 12, 6090-6097.

483 L. Li, M. Shalom, Y. Zhao, J. Barrio and M. Antonietti, J. Mater. Chem. A, 2017, 5, 18502-18508.

484 Z. Chen, M. Antonietti and D. Dontsova, Chem.-Eur. J., 2015, 21, 10805-10811.

485 L. Kui, X. Xin and Z. Wei-De, ChemCatChem, 2016, 8, 21282135.

486 Z. Mo, X. Zhu, Z. Jiang, Y. Song, D. Liu, H. Li, X. Yang, Y. She, Y. Lei, S. Yuan, H. Li, L. Song, Q. Yan and H. Xu, Appl. Catal., B, 2019, 256, 117854.

487 L. Kampschulte, M. Lackinger, A.-K. Maier, R. S. K. Kishore, S. Griessl, M. Schmittel and W. M. Heckl, J. Phys. Chem. B, 2006, 110, 10829-10836.

488 C. C. Robertson, J. S. Wright, E. J. Carrington, R. N. Perutz, C. A. Hunter and L. Brammer, Chem. Sci., 2017, 8, 53925398.

489 P. Li, H. D. Arman, H. Wang, L. Weng, K. Alfooty, R. F. Angawi and B. Chen, Cryst. Growth Des., 2015, 15, 1871-1875.

490 J. L. Cook, C. A. Hunter, C. M. R. Low, A. Perez-Velasco and J. G. Vinter, Angew. Chem., Int. Ed., 2007, 46, 3706-3709.

491 Z.-F. Huang, J. Song, L. Pan, Z. Wang, X. Zhang, J.-J. Zou, W. Mi, X. Zhang and L. Wang, Nano Energy, 2015, 12, 646-656.

492 J.-W. Zhang, S. Gong, N. Mahmood, L. Pan, X. Zhang and J.-J. Zou, Appl. Catal., B, 2018, 221, 9-16.

493 X. Weinan, C. Gang, L. Chunmei, S. Jingxue, H. Zhonghui, Z. Yansong, H. Yidong and M. Qingqiang, ChemCatChem, 2016, 8, 2838-2845. 
494 G. Peng, L. Xing, J. Barrio, M. Volokh and M. Shalom, Angew. Chem., Int. Ed., 2018, 57, 1186-1192.

495 L. Jiang, X. Yuan, G. Zeng, J. Liang, Z. Wu, H. Yu, D. Mo, H. Wang, Z. Xiao and C. Zhou, J. Colloid Interface Sci., 2019, 536, 17-29.

496 J. Barrio and M. Shalom, ACS Appl. Mater. Interfaces, 2018, 10, 39688-39694.

497 X. Hu, M. Vatankhah-Varnoosfaderani, J. Zhou, Q. Li and S. S. Sheiko, Adv. Mater., 2015, 27, 6899-6905.

498 L.-S. Teo, C.-Y. Chen and J.-F. Kuo, Macromolecules, 1997, 30, 1793-1799.

499 J. Wang, S. Cao and J. Yu, Sol. RRL, 2019, 1900435.

500 N. Karjule, J. Barrio, A. Tashakory and M. Shalom, Sol. RRL, 2020, 2000017.

501 J. Sun, J. Xu, A. Grafmueller, X. Huang, C. Liedel, G. AlgaraSiller, M. Willinger, C. Yang, Y. Fu, X. Wang and M. Shalom, Appl. Catal., B, 2017, 205, 1-10.

502 B. Qin, S. Zhang, Q. Song, Z. Huang, J.-F. Xu and X. Zhang, Angew. Chem., Int. Ed., 2017, 56, 7639-7643.

503 S. Dolai, J. Barrio, G. Peng, A. Grafmueller and M. Shalom, Nanoscale, 2019, 11, 5564-5570.

504 S. Dolai, N. Karjule, A. Azoulay and J. Barrio, RSC Adv., 2019, 9, 26091-26096.

505 R. S. Feigelson, in Handbook of Crystal Growth, Elsevier, 2015, pp. 1-83.

506 B. Spingler, S. Schnidrig, T. Todorova and F. Wild, CrystEngComm, 2012, 14, 751-757.

507 A. Azoulay, J. Barrio and M. Shalom, Isr. J. Chem., 2019, DOI: 10.1002/ijch.201900056.

508 J. Gao, J. Wang, X. Qian, Y. Dong, H. Xu, R. Song, C. Yan, H. Zhu, Q. Zhong, G. Qian and J. Yao, J. Solid State Chem., 2015, 228, 60-64.

509 J. Wang, H. Xu, X. Qian, Y. Dong, J. Gao, G. Qian and J. Yao, Chem.-Asian J., 2015, 10, 1276-1280.

510 J. Barrio, L. Lin, P. Amo-Ochoa, J. Tzadikov, G. Peng, J. Sun, F. Zamora, X. Wang and M. Shalom, Small, 2018, 14, 1800633.

511 D.-F. Weng, B.-W. Wang, Z.-M. Wang and S. Gao, CrystEngComm, 2011, 13, 4683-4688.

512 J.-N. Zhu, X.-Q. Zhu, F.-F. Cheng, P. Li, F. Wang, Y.-W. Xiao and W.-W. Xiong, Appl. Catal., B, 2019, 256, 117830.

513 Z. Jiang, X. Zhang, H. Chen, X. Hu and P. Yang, ChemCatChem, 2019, 11, 4558-4567.

514 X. Fang, R. Gao, Y. Yang and D. Yan, iScience, 2019, 16, 2230.

515 J.-H. Zhang, M.-J. Wei, Z.-W. Wei, M. Pan and C.-Y. Su, ACS Appl. Nano Mater., 2020, 3, 1010-1018.

516 Z. Liu, G. Wang, H.-S. Chen and P. Yang, Chem. Commun., 2018, 54, 4720-4723.

517 Z. Jiang, X. Zhang, J. Wang, L. Chen, H.-S. Chen and P. Yang, Chem. Commun., 2018, 54, 13519-13522.

518 J. Liu, Z. Wei, W. Fang, Z. Jiang and W. Shangguan, ChemCatChem, 2019, 11, 6275-6281.

519 J. Fu, B. Zhu, C. Jiang, B. Cheng, W. You and J. Yu, Small, 2017, 13, 1-9.

520 W. Ren, J. Cheng, H. Ou, C. Huang, M. Titirici and X. Wang, ChemSusChem, 2019, 12, 3257-3262.
521 M. Gao, J. Feng, Z. Zhang, M. Gu, J. Wang, W. Zeng, Y. Lv, Y. Ren, T. Wei and Z. Fan, ACS Appl. Nano Mater., 2018, 1, 6733-6741.

522 T. Sano, S. Tsutsui, K. Koike, T. Hirakawa, Y. Teramoto, N. Negishi and K. Takeuchi, J. Mater. Chem. A, 2013, 1, 6489-6496.

523 G. Dong, Z. Ai and L. Zhang, RSC Adv., 2014, 4, 5553-5560.

524 S. Liu, D. Li, H. Sun, H. M. Ang, M. O. Tadé and S. Wang, J. Colloid Interface Sci., 2016, 468, 176-182.

525 L. Xu, J. Xia, L. Wang, H. Ji, J. Qian, H. Xu, K. Wang and H. Li, Eur. J. Inorg. Chem., 2014, 2014, 3665-3673.

526 Q. Yang, Z. Li, C. Chen, Z. Zhang and X. Fang, RSC Adv., 2019, 9, 4404-4414.

527 W. Jiang, Q. Ruan, J. Xie, X. Chen, Y. Zhu and J. Tang, Appl. Catal., B, 2018, 236, 428-435.

528 Q. Han, B. Wang, J. Gao, Z. Cheng, Y. Zhao, Z. Zhang and L. Qu, ACS Nano, 2016, 10, 2745-2751.

529 Y. Xu, Q. Guo, L. Huang, H. Feng, C. Zhang, H. Xu and M. Wang, ACS Appl. Mater. Interfaces, 2019, 11, 3193431942.

530 Y. Zhang, A. Thomas, M. Antonietti and X. Wang, J. Am. Chem. Soc., 2008, 131, 50-51.

531 C. Ye, X.-Z. Wang, J.-X. Li, Z.-J. Li, X.-B. Li, L.-P. Zhang, B. Chen, C.-H. Tung and L.-Z. Wu, ACS Catal., 2016, 6, 8336-8341.

532 T. Y. Ma, Y. Tang, S. Dai and S. Z. Qiao, Small, 2014, 10, 2382-2389.

533 X. Qu, S. Hu, J. Bai, P. Li, G. Lu and X. Kang, New J. Chem., 2018, 42, 4998-5004.

534 D. K. Chauhan, S. Jain, V. R. Battula and K. Kailasam, Carbon, 2019, 152, 40-58.

535 Y. Guo, S. Chu, S. Yan, Y. Wang and Z. Zou, Chem. Commun., 2010, 46, 7325-7327.

536 G. Dong, L. Yang, F. Wang, L. Zang and C. Wang, ACS Catal., 2016, 6, 6511-6519.

537 J. Hu, D. Chen, N. Li, Q. Xu, H. Li, J. He and J. Lu, Small, 2018, 14, 1800416.

538 V. W. Lau, I. Moudrakovski, T. Botari, S. Weinberger, M. B. Mesch, V. Duppel, J. Senker, V. Blum and B. V Lotsch, Nat. Commun., 2016, 7, 12165.

539 G. Liu, G. Zhao, W. Zhou, Y. Liu, H. Pang, H. Zhang, D. Hao, X. Meng, P. Li, T. Kako and J. Ye, Adv. Funct. Mater., 2016, 26, 6822-6829.

540 L. Li, D. Cruz, A. Savateev, G. Zhang, M. Antonietti and Y. Zhao, Appl. Catal., B, 2018, 229, 249-253.

541 V. W. Lau, V. W. Yu, F. Ehrat, T. Botari, I. Moudrakovski, T. Simon, V. Duppel, E. Medina, J. K. Stolarczyk, J. Feldmann, V. Blum and B. V Lotsch, Adv. Energy Mater., 2017, 7, 1602251.

542 X. Fan, L. Zhang, M. Wang, W. Huang, Y. Zhou, M. Li, R. Cheng and J. Shi, Appl. Catal., B, 2016, 182, 68-73.

543 J. Tian, L. Zhang, X. Fan, Y. Zhou, M. Wang, R. Cheng, M. Li, X. Kan, X. Jin, Z. Liu, Y. Gao and J. Shi, J. Mater. Chem. A, 2016, 4, 13814-13821.

544 D. Vidyasagar, S. G. Ghugal, S. S. Umare and M. Banavoth, Sci. Rep., 2019, 9, 7186. 
545 J. Pan, L. Guo, S. Zhang, N. Wang, S. Jin and B. Tan, Chem.Asian J., 2018, 13, 1674-1677.

546 W. Lu, T. Xu, Y. Wang, H. Hu, N. Li, X. Jiang and W. Chen, Appl. Catal., B, 2016, 180, 20-28.

547 G. Zhao, H. Pang, G. Liu, P. Li, H. Liu, H. Zhang, L. Shi and J. Ye, Appl. Catal., B, 2017, 200, 141-149.

548 S. Tian, S. Chen, X. Ren, R. Cao, H. Hu and F. Bai, Nano Res., 2019, 12, 3109-3115.

549 S. Zhao, T. Guo, X. Li, T. Xu, B. Yang and X. Zhao, Appl. Catal., B, 2018, 224, 725-732.

550 P. Zhang, H. Li and Y. Wang, Chem. Commun., 2014, 50, 6312-6315.

551 X. Chen, H. Chen, J. Guan, J. Zhen, Z. Sun, P. Du, Y. Lu and S. Yang, Nanoscale, 2017, 9, 5615-5623.

552 X.-H. Song, L. Feng, S.-L. Deng, S.-Y. Xie and L.-S. Zheng, Adv. Mater. Interfaces, 2017, 4, 1700339.

553 A. Rashidizadeh, H. Ghafuri, H. Reza Esmaili Zand and N. Goodarzi, ACS Omega, 2019, 4, 12544-12554.

554 Y.-L. Thi Ngo, J. S. Chung and S. H. Hur, Dyes Pigm., 2019, 168, 180-188.

555 W. S. Hummers and R. E. Offeman, J. Am. Chem. Soc., 2002, 80, 1339.

556 J. Sun, R. Phatake, A. Azoulay, G. Peng, C. Han, J. Barrio, J. Xu, X. Wang and M. Shalom, Chem.-Eur. J., 2018, 24, 14921-14927.

557 B. Kumru, M. Antonietti and B. V. K. J. Schmidt, Langmuir, 2017, 33, 9897-9906.

558 B. Kumru, J. Barrio, J. Zhang, M. Antonietti, M. Shalom and B. V. K. J. Schmidt, ACS Appl. Mater. Interfaces, 2019, 11, 9462-9469.

559 M. A. Douzandegi Fard, H. Ghafuri and A. Rashidizadeh, Microporous Mesoporous Mater., 2019, 274, 83-93.

560 B. Kumru, V. Molinari, M. Hilgart, F. Rummel, M. Schäffler and B. V. K. J. Schmidt, Polym. Chem., 2019, 10, 3647-3656.

561 Q. Cao, B. Kumru, M. Antonietti and B. V. K. J. Schmidt, Macromolecules, 2019, 52, 4989-4996.

562 Q. Cao, B. Kumru, M. Antonietti and B. V. K. J. Schmidt, Mater. Horiz., 2020, 7, 762-786.

563 C. Hu, Y.-R. Lin and H.-C. Yang, ChemSusChem, 2019, 12, 1794-1806.

564 M. Li, H. Liao, Q. Deng, Y. Wu, F. Xiao, X. Wei and D. Tu, J. Macromol. Sci., Part A: Pure Appl.Chem., 2018, 55, 408-413.

565 M. Zarei, H. Ahmadzadeh, E. K. Goharshadi and A. Farzaneh, Anal. Chim. Acta, 2015, 887, 245-252.

566 J. Wan Ko, W. Seok Choi, J. Kim, S. Keun Kuk, S. Ha Lee and C. Beum Park, Biomacromolecules, 2017, 18, 3551-3556.

567 J. Yan, M.-T. F. Rodrigues, Z. Song, H. Li, H. Xu, H. Liu, J. Wu, Y. Xu, Y. Song, Y. Liu, P. Yu, W. Yang, R. Vajtai, H. Li, S. Yuan and P. M. Ajayan, Adv. Funct. Mater., 2017, 27, 1700653.

568 X. Wang, Y. Liang, W. An, J. Hu, Y. Zhu and W. Cui, Appl. Catal., B, 2017, 219, 53-62.

569 L. Tang, C. Jia, Y. Xue, L. Li, A. Wang, G. Xu, N. Liu and M. Wu, Appl. Catal., B, 2017, 219, 241-248.

570 Q. Han, Z. Cheng, J. Gao, Y. Zhao, Z. Zhang, L. Dai and L. Qu, Adv. Funct. Mater., 2017, 27, 1606352.
571 Z. Tong, D. Yang, J. Shi, Y. Nan, Y. Sun and Z. Jiang, ACS Appl. Mater. Interfaces, 2015, 7, 25693-25701.

572 M. Zhang, W. Jiang, D. Liu, J. Wang, Y. Liu, Y. Zhu and Y. Zhu, Appl. Catal., B, 2016, 183, 263-268.

573 Y. Zhang, Z. Zhou, Y. Shen, Q. Zhou, J. Wang, A. Liu, S. Liu and Y. Zhang, ACS Nano, 2016, 10, 9036-9043.

574 W. Jiang, W. Luo, R. Zong, W. Yao, Z. Li and Y. Zhu, Small, 2016, 12, 4370-4378.

575 J. Sun, B. V. K. J. Schmidt, X. Wang and M. Shalom, ACS Appl. Mater. Interfaces, 2017, 9, 2029-2034.

576 J. Liu, T. An, Z. Chen, Z. Wang, H. Zhou, T. Fan, D. Zhang and M. Antonietti, J. Mater. Chem. A, 2017, 5, 8933-8938.

577 B. Kumru, M. Shalom, M. Antonietti and B. V. K. J. Schmidt, Macromolecules, 2017, 50, 1862-1869.

578 B. Kumru, V. Molinari, R. Dünnebacke, K. G. Blank and B. V. K. J. Schmidt, Macromol. Rapid Commun., 2019, 40, 1800712.

579 B. Kumru, V. Molinari, M. Shalom, M. Antonietti and B. V. K. J. Schmidt, Soft Matter, 2018, 14, 2655-2664.

580 B. Ye, C. Yao, M. Yan, H. Zhang, F. Xi, J. Liu, B. Li and X. Dong, Macromol. Mater. Eng., 2019, 304, 1800500.

581 J. N. Coleman, M. Lotya, A. O'Neill, S. D. Bergin, P. J. King, U. Khan, K. Young, A. Gaucher, S. De, R. J. Smith, I. V Shvets, S. K. Arora, G. Stanton, H.-Y. Kim, K. Lee, G. T. Kim, G. S. Duesberg, T. Hallam, J. J. Boland, J. J. Wang, J. F. Donegan, J. C. Grunlan, G. Moriarty, A. Shmeliov, R. J. Nicholls, J. M. Perkins, E. M. Grieveson, K. Theuwissen, D. W. McComb, P. D. Nellist and V. Nicolosi, Science, 2011, 331, 568-571.

582 V. Nicolosi, M. Chhowalla, M. G. Kanatzidis, M. S. Strano and J. N. Coleman, Science, 2013, 340, 1226419.

583 Y. Hernandez, V. Nicolosi, M. Lotya, F. M. Blighe, Z. Sun, S. De, I. T. McGovern, B. Holland, M. Byrne, Y. K. Gun'Ko, J. J. Boland, P. Niraj, G. Duesberg, S. Krishnamurthy, R. Goodhue, J. Hutchison, V. Scardaci, A. C. Ferrari and J. N. Coleman, Nat. Nanotechnol., 2008, 3, 563-568.

584 Y. Lin, T. V. Williams and J. W. Connell, J. Phys. Chem. Lett., 2009, 1, 277-283.

585 M. Chhowalla, H. S. Shin, G. Eda, L.-J. Li, K. P. Loh and H. Zhang, Nat. Chem., 2013, 5, 263-275.

586 M. Pumera and Z. Sofer, Adv. Mater., 2017, 29, 1605299.

587 Y. Niu, J. Villalva, R. Frisenda, G. Sanchez-Santolino, L. Ruiz-González, E. M. Pérez, M. García-Hernández, E. Burzurí and A. Castellanos-Gomez, 2D Mater., 2019, 6, 35023.

588 C. Gibaja, D. Rodriguez-San-Miguel, P. Ares, J. GómezHerrero, M. Varela, R. Gillen, J. Maultzsch, F. Hauke, A. Hirsch, G. Abellán and F. Zamora, Angew. Chem., Int. Ed., 2016, 55, 14345-14349.

589 J. Zhang, Y. Chen and X. Wang, Energy Environ. Sci., 2015, 8, 3092-3108.

590 Z. Qin, Z. Huang, M. Wang, D. Liu, Y. Chen and L. Guo, Appl. Catal., B, 2020, 261, 118211.

591 X. Zhang, X. Xie, H. Wang, J. Zhang, B. Pan and Y. Xie, J. Am. Chem. Soc., 2012, 135, 18-21. 
592 S. Yang, Y. Gong, J. Zhang, L. Zhan, L. Ma, Z. Fang, R. Vajtai, X. Wang and P. M. Ajayan, Adv. Mater., 2013, 25, 2452-2456.

593 X. She, H. Xu, Y. Xu, J. Yan, J. Xia, L. Xu, Y. Song, Y. Jiang, Q. Zhang and H. Li, J. Mater. Chem. A, 2014, 2, 2563-2570.

594 C. Huang, J. Wen, Y. Shen, F. He, L. Mi, Z. Gan, J. Ma, S. Liu, H. Ma and Y. Zhang, Chem. Sci., 2018, 9, 7912-7915.

595 Z. Gan, C. Huang, Y. Shen, Q. Zhou, D. Han, J. Ma, S. Liu and Y. Zhang, Chin. Chem. Lett., 2020, 31, 513-516.

596 X. Bai, S. Yan, J. Wang, L. Wang, W. Jiang, S. Wu, C. Sun and Y. Zhu, J. Mater. Chem. A, 2014, 2, 17521-17529.

597 Q. Lin, L. Li, S. Liang, M. Liu, J. Bi and L. Wu, Appl. Catal., B, 2015, 163, 135-142.

598 S. P. Pattnaik, A. Behera, S. Martha, R. Acharya and K. Parida, J. Mater. Sci., 2019, 54, 5726-5742.

599 H. Zhang, L.-H. Guo, L. Zhao, B. Wan and Y. Yang, J. Phys. Chem. Lett., 2015, 6, 958-963.

600 Z. Zhou, Q. Shang, Y. Shen, L. Zhang, Y. Zhang, Y. Lv, Y. Li, S. Liu and Y. Zhang, Anal. Chem., 2016, 88, 6004-6010.

601 P. Qiu, H. Chen, C. Xu, N. Zhou, F. Jiang, X. Wang and Y. Fu, J. Mater. Chem. A, 2015, 3, 24237-24244.

602 S. Guo, Y. Zhu, Y. Yan, Y. Min, J. Fan and Q. Xu, Appl. Catal., $B, 2016,185,315-321$.

603 P. Niu, L. Zhang, G. Liu and H.-M. Cheng, Adv. Funct. Mater., 2012, 22, 4763-4770.

604 P. Xia, B. Zhu, J. Yu, S. Cao and M. Jaroniec, J. Mater. Chem. A, 2017, 5, 3230-3238.

605 J. Xu, L. Zhang, R. Shi and Y. Zhu, J. Mater. Chem. A, 2013, 1, 14766.

606 Z. Zhou, Y. Shen, Y. Li, A. Liu, S. Liu and Y. Zhang, ACS Nano, 2015, 9, 12480-12487.

607 Z. Zhou, J. Wang, J. Yu, Y. Shen, Y. Li, A. Liu, S. Liu and Y. Zhang, J. Am. Chem. Soc., 2015, 137, 2179-2182.

608 M. Yi and Z. Shen, J. Mater. Chem. A, 2015, 3, 11700-11715. 609 H. Li, G. Lu, Y. Wang, Z. Yin, C. Cong, Q. He, L. Wang, F. Ding, T. Yu and H. Zhang, Small, 2013, 9, 1974-1981.

610 A. Castellanos-Gomez, L. Vicarelli, E. Prada, J. O. Island, K. L. Narasimha-Acharya, S. I. Blanter, D. J. Groenendijk, M. Buscema, G. A. Steele, J. V. Alvarez, H. W. Zandbergen, J. J. Palacios and H. S. J. van der Zant, 2D Mater., 2014, 1, 025001.

611 P. Ares, F. Aguilar-Galindo, D. Rodríguez-San-Miguel, D. A. Aldave, S. Díaz-Tendero, M. Alcamí, F. Martín, J. Gómez-Herrero and F. Zamora, Adv. Mater., 2016, 28, 6332-6336.

612 M. J. Bojdys, N. Severin, J. P. Rabe, A. I. Cooper, A. Thomas and M. Antonietti, Macromol. Rapid Commun., 2013, 34, 850-854.

613 K. Schwinghammer, M. B. Mesch, V. Duppel, C. Ziegler, J. Senker and B. V. Lotsch, J. Am. Chem. Soc., 2014, 136, 1730-1733.

614 T. S. Miller, T. M. Suter, A. M. Telford, L. Picco, O. D. Payton, F. Russell-Pavier, P. L. Cullen, A. Sella, M. S. P. Shaffer, J. Nelson, V. Tileli, P. F. McMillan and C. A. Howard, Nano Lett., 2017, 17, 5891-5896.

615 H. Xu, J. Yan, X. She, L. Xu, J. Xia, Y. Xu, Y. Song, L. Huang and H. Li, Nanoscale, 2014, 6, 1406-1415.
616 L. Ma, H. Fan, M. Li, H. Tian, J. Fang and G. Dong, J. Mater. Chem. A, 2015, 3, 22404-22412.

617 J. Ji, J. Wen, Y. Shen, Y. Lv, Y. Chen, S. Liu, H. Ma and Y. Zhang, J. Am. Chem. Soc., 2017, 139, 11698-11701.

618 Y. Li, S. Ouyang, H. Xu, X. Wang, Y. Bi, Y. Zhang and J. Ye, J. Am. Chem. Soc., 2016, 138, 13289-13297.

619 Y. Li, S. Ouyang, H. Xu, W. Hou, M. Zhao, H. Chen and J. Ye, Adv. Funct. Mater., 2019, 29, 1901024.

620 Š. Hajduk, S. P. Berglund, M. Podlogar, G. Dražić, F. F. Abdi, Z. C. Orel and M. Shalom, Adv. Mater. Interfaces, 2017, 4, 1700924.

621 W.-J. Ong, Front. Mater., 2017, 4, 1-10.

622 J. Barrio, C. Gibaja, J. Tzadikov, M. Shalom and F. Zamora, Adv. Sustainable Syst., 2019, 3, 1800138.

623 A. Bafaqeer, M. Tahir and N. A. S. Amin, Appl. Catal., B, 2019, 242, 312-326.

624 Z. Gao, K. Chen, L. Wang, B. Bai, H. Liu and Q. Wang, Appl. Catal., B, 2019, 118462.

625 M. S. Khan, F. Zhang, M. Osada, S. S. Mao and S. Shen, Sol. $R R L, 2019,1900435$.

626 J. Low, J. Yu, M. Jaroniec, S. Wageh and A. A. Al-Ghamdi, Adv. Mater., 2017, 29, 1601694.

627 S. Yin, J. Di, M. Li, Y. Sun, J. Xia, H. Xu, W. Fan and H. Li, J. Mater. Sci., 2016, 51, 4769-4777.

628 S. Han, X. Hu, W. Yang, Q. Qian, X. Fang and Y. Zhu, ACS Appl. Energy Mater., 2019, 2, 1803-1811.

629 W. Zhang, A. R. Mohamed and W.-J. Ong, Angew. Chem., Int. Ed., 2020, DOI: 10.1002/anie.201914925.

630 J. Fu, J. Yu, C. Jiang and B. Cheng, Adv. Energy Mater., 2018, 8, 1-31.

631 H. Xu, X. She, T. Fei, Y. Song, D. Liu, H. Li, X. Yang, J. Yang, H. Li, L. Song, P. M. Ajayan and J. Wu, ACS Nano, 2019, 13, 11294-11302.

632 L. Sun, B. Li, X. Chu, N. Sun, Y. Qu, X. Zhang, I. Khan, L. Bai and L. Jing, ACS Sustainable Chem. Eng., 2019, 7, 9916-9927.

633 R. Bhosale, S. Jain, C. P. Vinod, S. Kumar and S. Ogale, ACS Appl. Mater. Interfaces, 2019, 11, 6174-6183.

634 T. Di, B. Zhu, B. Cheng, J. Yu and J. Xu, J. Catal., 2017, 352, 532-541.

635 Z. Pan, G. Zhang and X. Wang, Angew. Chem., Int. Ed., 2019, 58, 7102-7106.

636 K. Zhu, M. Zhang, X. Feng, L. Qin, S.-Z. Kang and X. Li, Appl. Catal., B, 2019, 118434.

637 X.-H. Li and M. Antonietti, Chem. Soc. Rev., 2013, 42, 6593. 638 J. Wang, G. Wang, X. Wang, Y. Wu, Y. Su and H. Tang, Carbon, 2019, 149, 618-626.

639 Q. Han, N. Chen, J. Zhang and L. Qu, Mater. Horiz., 2017, 4, 832-850.

640 C. Wang, G. Liu, K. Song, X. Wang, H. Wang, N. Zhao and F. He, ChemCatChem, 2019, 11, 6364-6371.

641 Y. Li, H. Zhang, P. Liu, D. Wang, Y. Li and H. Zhao, Small, 2013, 9, 3336-3344.

642 X. Chen, K. Deng, P. Zhou and Z. Zhang, ChemSusChem, 2018, 11, 2444-2452.

643 J. Ran, W. Guo, H. Wang, B. Zhu, J. Yu and S.-Z. Qiao, Adv. Mater., 2018, 30, 1800128. 
644 C.-C. Wang, X.-H. Yi and P. Wang, Appl. Catal., B, 2019, 247, 24-48.

645 P. Kumar, P. Kar, A. P. Manuel, S. Zeng, U. K. Thakur, K. M. Alam, Y. Zhang, R. Kisslinger, K. Cui, G. M. Bernard, V. K. Michaelis and K. Shankar, Adv. Opt. Mater., 2019, 8, 1901275.

646 Y. Zhu, T. Wan, X. Wen, D. Chu and Y. Jiang, Appl. Catal., B, 2019, 244, 814-822.

647 W. Chang, W. Xue, E. Liu, J. Fan and B. Zhao, Chem. Eng. J., 2019, 362, 392-401.

648 C. He, J. H. Zhang, W. X. Zhang and T. T. Li, J. Phys. Chem. Lett., 2019, 10, 3122-3128.

649 P. Chen, F. Liu, H. Ding, S. Chen, L. Chen, Y.-J. Li, C.-T. Au and S.-F. Yin, Appl. Catal., B, 2019, 252, 33-40.

650 C. A. Caputo, L. Wang, R. Beranek and E. Reisner, Chem. Sci., 2015, 6, 5690-5694.

651 C. A. Caputo, M. A. Gross, V. W. Lau, C. Cavazza, B. V. Lotsch and E. Reisner, Angew. Chem., Int. Ed., 2014, 53, 11538-11542.

652 C. Li, S. Yu, L. Gu, J. Han, H. Dong, Y. Wang and G. Chen, Adv. Mater. Interfaces, 2018, 5, 1801297.

653 H. Zhang, J. Wu, J. Han, L. Wang, W. Zhang, H. Dong, C. Li and Y. Wang, Chem. Eng. J., 2020, 385, 123764.

654 H. Kasap, C. A. Caputo, B. C. M. Martindale, R. Godin, V. W. Lau, B. V. Lotsch, J. R. Durrant and E. Reisner, J. Am. Chem. Soc., 2016, 138, 9183-9192.

655 Z. Pan, P. Niu, M. Liu, G. Zhang, Z. Zhu and X. Wang, ChemSusChem, 2020, 13, 888-892.

656 S. Ding, M. J. Hülsey, J. Pérez-Ramírez and N. Yan, Joule, 2019, 3, 2897-2929.

657 Z. Chen, S. Mitchell, F. Krumeich, R. Hauert, S. Yakunin, M. V. Kovalenko and J. Pérez-Ramírez, ACS Sustainable Chem. Eng., 2019, 7, 5223-5230.

658 P. Huang, J. Huang, S. A. Pantovich, A. D. Carl, T. G. Fenton, C. A. Caputo, R. L. Grimm, A. I. Frenkel and G. Li, J. Am. Chem. Soc., 2018, 140, 16042-16047.

659 G. Gao, Y. Jiao, E. R. Waclawik and A. Du, J. Am. Chem. Soc., 2016, 138, 6292-6297.

660 G. Vilé, D. Albani, M. Nachtegaal, Z. Chen, D. Dontsova, M. Antonietti, N. López and J. Pérez-Ramírez, Angew. Chem., Int. Ed., 2015, 54, 11265-11269.

661 L. Zhang, W. Zhao, W. Zhang, J. Chen and Z. Hu, Nano Res., 2019, 12, 1181-1186.

662 Z. Chen, J. Zhao, C. R. Cabrera and Z. Chen, Small Methods, 2019, 3, 1800368.

663 X. Wang, X. Chen, A. Thomas, X. Fu and M. Antonietti, Adv. Mater., 2009, 21, 1609-1612.

664 G. Zhang, G. Li and X. Wang, ChemCatChem, 2015, 7, 28642870.

665 C. Liao, B. Yang, N. Zhang, M. Liu, G. Chen, X. Jiang, G. Chen, J. Yang, X. Liu, T. S. Chan, Y. J. Lu, R. Ma and W. Zhou, Adv. Funct. Mater., 2019, 29, 1-11.

666 K. Wang, D. Shao, L. Zhang, Y. Zhou, H. Wang and W. Wang, J. Mater. Chem. A, 2019, 7, 20383-20389.

667 Y. Peng, W. Pan, N. Wang, J.-E. Lu and S. Chen, ChemSusChem, 2018, 11, 130-136.
668 J. Barrio, D. Mateo, J. Albero, H. García and M. Shalom, Adv. Energy Mater., 2019, 9, 1902738.

669 K. Li, Y. Zhang, Y.-Z. Lin, K. Wang and F. Liu, ACS Appl. Mater. Interfaces, 2019, 11, 28918-28927.

670 W. Ye, J. Hu, X. Hu, W. Zhang, X. Ma and H. Wang, ChemCatChem, 2019, 11, 6372-6383.

671 Y. Wang, N. Zhang, R. Hübner, D. Tan, M. Löffler, S. Facsko, E. Zhang, Y. Ge, Z. Qi and C. Wu, Adv. Mater. Interfaces, 2019, 6, 1801664.

672 W. Xiong, F. Huang and R.-Q. Zhang, Sustainable Energy Fuels, 2020, 4, 485-503.

673 Y. Luo, Y. Yan, S. Zheng, H. Xue and H. Pang, J. Mater. Chem. A, 2019, 7, 901-924.

674 W. Niu and Y. Yang, ACS Energy Lett., 2018, 3, 2796-2815.

675 J. Bian, L. Xi, C. Huang, K. M. Lange, R. Q. Zhang and M. Shalom, Adv. Energy Mater., 2016, 6, 4-9.

676 Q. Ruan, M. K. Bayazit, V. Kiran, J. Xie, Y. Wang and J. Tang, Chem. Commun., 2019, 55, 7191-7194.

677 L. Zhao, J. Ji, Y. Shen, K. Wu, T. Zhao, H. Yang, Y. Lv, S. Liu and Y. Zhang, Chem.-Eur. J., 2019, 25, 15680-15686.

678 F. Podjaski, J. Kröger and B. V. Lotsch, Adv. Mater., 2018, 30, 1705477.

679 N. A. Mohamed, H. Ullah, J. Safaei, A. F. Ismail, M. F. Mohamad Noh, M. F. Soh, M. A. Ibrahim, N. A. Ludin and M. A. Mat Teridi, J. Phys. Chem. C, 2019, 123, 9013-9026.

680 M. Sima, E. Vasile, A. Sima, N. Preda and C. Logofatu, Int. J. Hydrogen Energy, 2019, 44, 24430-24440.

681 C. Ott, F. Reiter, M. Baumgartner, M. Pielmeier, A. Vogel, P. Walke, S. Burger, M. Ehrenreich, G. Kieslich, D. Daisenberger, J. Armstrong, U. K. Thakur, P. Kumar, S. Chen, D. Donadio, L. S. Walter, R. T. Weitz, K. Shankar and T. Nilges, Adv. Funct. Mater., 2019, 29, 1900233.

682 B. Kumru, D. Cruz, T. Heil, B. V. K. J. Schmidt and M. Antonietti, J. Am. Chem. Soc., 2018, 140, 17532-17537.

683 D. Cruz, J. Garcia Cerrillo, B. Kumru, N. Li, J. Dario Perea, B. V. K. J. Schmidt, I. Lauermann, C. J. Brabec and M. Antonietti, J. Am. Chem. Soc., 2019, 141, 12322-12328.

684 Z. Li, S. Wu, J. Zhang, Y. Yuan, Z. Wang and Z. Zhu, Sol. $R R L, 2019,4,1900413$.

685 W. Cao, K. Lin, J. Li, L. Qiu, Y. Dong, J. Wang, D. Xia, R. Fan and Y. Yang, J. Mater. Chem. C, 2019, 7, 12717-12724.

686 C. Murugan, K. B. Bhojanaa, W.-J. Ong, K. Jothivenkatachalam and A. Pandikumar, Int. J. Hydrogen Energy, 2019, 44, 30885-30898.

687 Q. Cao, B. Kumru, M. Antonietti and B. V. K. J. Schmidt, Mater. Horiz., 2020, 7, 762-786.

688 N. Karjule, R. Phatake, M. Volokh, I. Hod and M. Shalom, Small Methods, 2019, 3, 1900401.

689 J. Xu, S. Cao, T. Brenner, X. Yang, J. Yu, M. Antonietti and M. Shalom, Adv. Funct. Mater., 2015, 25, 6265-6271.

690 X. Fan, T. Wang, B. Gao, H. Gong, H. Xue, H. Guo, L. Song, W. Xia, X. Huang and J. He, Langmuir, 2016, 32, 1332213332.

691 X. Xie, X. Fan, X. Huang, T. Wang and J. He, RSC Adv., 2016, 6, 9916-9922. 
692 Y. Xiao, M. Chen and M. Zhang, ChemPhotoChem, 2019, 3, 101-106.

693 G. Peng, M. Volokh, J. Tzadikov, J. Sun and M. Shalom, Adv. Energy Mater., 2018, 8, 1-7.

694 G. Peng, J. Qin, M. Volokh, C. Liu and M. Shalom, J. Mater. Chem. A, 2019, 7, 11718-11723.

695 R. Godin, Y. Wang, M. A. Zwijnenburg, J. Tang and J. R. Durrant, J. Am. Chem. Soc., 2017, 139, 5216-5224.

696 G. Peng, J. Albero, H. Garcia and M. Shalom, Angew. Chem., Int. Ed., 2018, 57, 15807-15811.

697 X. Lv, M. Cao, W. Shi, M. Wang and Y. Shen, Carbon, 2017, 117, 343-350.

698 M. Huang, Y.-L. Zhao, W. Xiong, S. V. Kershaw, Y. Yu, W. Li, T. Dudka and R.-Q. Zhang, Appl. Catal., B, 2018, 237, 783790.

699 P. Luan, Q. Meng, J. Wu, Q. Li, X. Zhang, Y. Zhang, L. A. O'Dell, S. R. Raga, J. Pringle, J. C. Griffith, C. Sun, U. Bach and J. Zhang, ChemSusChem, 2020, 13, 328-333.

700 H. Li, H. Huang, Z. Wang, Z. Zheng, P. Wang, Y. Liu, X. Zhang, X. Qin, Y. Dai, Y. Li, H. Zou and B. Huang, Catal. Today, 2020, 340, 92-96.

701 W. Xiong, S. Chen, M. Huang, Z. Wang, Z. Lu and R. Zhang, ChemSusChem, 2018, 11, 2497-2501.

702 K. Xiao, B. Tu, L. Chen, T. Heil, L. Wen, L. Jiang and M. Antonietti, Angew. Chem., Int. Ed., 2019, 58, 1257412579.

703 K. Xiao, L. Chen, R. Chen, T. Heil, S. D. C. Lemus, F. Fan, L. Wen, L. Jiang and M. Antonietti, Nat. Commun., 2019, 10, 74 .

704 J. Albero, E. M. Barea, J. Xu, I. Mora-Seró, H. Garcia and M. Shalom, Adv. Mater. Interfaces, 2017, 4, 1600265.
705 Y. Li, G. Liu, D. Jia, C. Li, L. Wang, J. Zheng, X. Liu and Z. Jiao, Catal. Lett., 2019, 149, 19-24.

706 D.-D. Qin, J.-J. Quan, S.-F. Duan, J. San Martin, Y. Lin, X. Zhu, X.-Q. Yao, J.-Z. Su, I. Rodríguez-Gutiérrez, C.-L. Tao and Y. Yan, ChemSusChem, 2019, 12, 898-907.

707 Y. Yang, Q. Zhang, R. Zhang, T. Ran, W. Wan and Y. Zhou, Front. Chem., 2018, 6, 1-10.

708 F. Jia, Y. Zhang, W. Hu, M. Lv, C. Jia and J. Liu, Front. Mater., 2019, 6, 1-9.

709 G. Peng, J. Qin, M. Volokh and M. Shalom, ACS Appl. Mater. Interfaces, 2019, 11, 29139-29146.

710 S. Otep, T. Michinobu and Q. Zhang, Sol. RRL, 2019, 1900395.

711 K. Sivula and R. van de Krol, Nat. Rev. Mater., 2016, 1, 15010.

712 I. Roger, M. A. Shipman and M. D. Symes, Nat. Rev. Chem., 2017, 1, 0003.

713 K. S. Lakhi, D.-H. Park, K. Al-Bahily, W. Cha, B. Viswanathan, J.-H. Choy and A. Vinu, Chem. Soc. Rev., 2017, 46, 72-101.

714 Q. Wang and K. Domen, Chem. Rev., 2020, 120, 919-985.

715 Y. Goto, T. Hisatomi, Q. Wang, T. Higashi, K. Ishikiriyama, T. Maeda, Y. Sakata, S. Okunaka, H. Tokudome, M. Katayama, S. Akiyama, H. Nishiyama, Y. Inoue, T. Takewaki, T. Setoyama, T. Minegishi, T. Takata, T. Yamada and K. Domen, Joule, 2018, 2, 509-520.

716 T. Takata and K. Domen, ACS Energy Lett., 2019, 542-549. 717 Q. Wang, T. Hisatomi, Q. Jia, H. Tokudome, M. Zhong, C. Wang, Z. Pan, T. Takata, M. Nakabayashi, N. Shibata, Y. Li, I. D. Sharp, A. Kudo, T. Yamada and K. Domen, Nat. Mater., 2016, 15, 611-615. 